\title{
A complete catalogue of dusty supernova remnants in the Galactic plane
}

\author{
H. Chawner, ${ }^{1 \star}$ H. L. Gomez, ${ }^{1 \star}$ M. Matsuura ${ }^{\oplus},{ }^{1 \star}$ M. W. L. Smith ${ }^{\oplus},{ }^{1}$ \\ A. Papageorgiou, ${ }^{1}$ J. Rho, ${ }^{2,3}$ A. Noriega-Crespo, ${ }^{4}$ I. De Looze ${ }^{\oplus},{ }^{5,6}$ M. J. Barlow ${ }^{\oplus}, 5$ \\ P. Cigan, ${ }^{1}$ L. Dunne ${ }^{1}$ and K. Marsh ${ }^{\oplus 7}$ \\ ${ }^{1}$ School of Physics and Astronomy, Cardiff University, Queens Buildings, The Parade, Cardiff CF24 3AA, UK \\ ${ }^{2}$ SETI Institute, 189 N. Bernardo Ave, Suite 100, Mountain View, CA 94043, USA \\ ${ }^{3}$ SOFIA Science Center, NASA Ames Research Center, MS 232, Moffett Field, CA 94035, USA \\ ${ }^{4}$ Space Telescope Science Institute, 3700 San Martin Drive, Baltimore, MD 21218, USA \\ ${ }^{5}$ Department of Physics and Astronomy, University College London, Gower Street, London WC1E 6BT, UK \\ ${ }^{6}$ Sterrenkundig Observatorium, Ghent University, Krijgslaan 281 - S9, B-9000 Ghent, Belgium \\ ${ }^{7}$ IPAC, Caltech, 1200 E California Blvd, Pasadena, CA 91125, USA
}

Accepted 2020 January 10. Received 2019 November 1; in original form 2019 March 18

\begin{abstract}
We search for far-infrared (FIR) counterparts of known supernova remnants (SNRs) in the Galactic plane $\left(360^{\circ}\right.$ in longitude and $\left.b= \pm 1^{\circ}\right)$ at $70-500 \mu \mathrm{m}$ with Herschel. We detect dust signatures in 39 SNRs out of 190, made up of 13 core-collapse supernovae (CCSNe), including 4 Pulsar Wind Nebulae (PWNe), and 2 Type Ia SNe. A further 24 FIR detected SNRs have unknown types. We confirm the FIR detection of ejecta dust within G350.1-0.3, adding to the known sample of $\sim 10$ SNRs containing ejecta dust. We discover dust features at the location of a radio core at the centre of G351.2+0.1, indicating FIR emission coincident with a possible Crab-like compact object, with dust temperature and mass of $T_{d}=45.8 \mathrm{~K}$ and $M_{d}$ $=0.18 \mathrm{M}_{\odot}$, similar to the PWN G54.1+0.3. We show that the detection rate is higher among young SNRs. We produce dust temperature maps of 11 SNRs and mass maps of those with distance estimates, finding dust at temperatures $15 \lesssim T_{d} \lesssim 40 \mathrm{~K}$. If the dust is heated by shock interactions the shocked gas must be relatively cool and/or have a low density to explain the observed low grain temperatures.
\end{abstract}

Key words: ISM: supernova remnants - infrared: ISM - submillimetre: ISM.

\section{INTRODUCTION}

Whether or not supernovae are an important contributor to the dust budget of galaxies at all cosmic epochs remains an unresolved question (Morgan \& Edmunds 2003; Dwek, Galliano \& Jones 2007; Matsuura et al. 2009; Gall, Hjorth \& Andersen 2011; Rowlands et al. 2014; Mancini et al. 2015; De Vis et al. 2017). One way to resolve this is to search for infrared (IR) and submillimetre (submm) emission from cold ejecta dust in nearby supernovae ( $\mathrm{SNe}$ ) and supernova remnants (SNR), where the sources are close enough to resolve dust structures in the FIR. There now exists a handful of individual SNRs with reported detections of SN-associated dust structures, containing significant quantities of dust. These include Cassiopeia A (Dunne et al. 2003; Krause et al. 2004; Dunne et al. 2009; Barlow et al. 2010; De Looze et al. 2017), the Crab Nebula (Gomez et al. 2012b; Temim et al. 2012; De Looze et al. 2019), SNR1987A (Indebetouw et al. 2014; Matsuura et al. 2015),

^E-mail: chawnerhs@ @ardiff.ac.uk (HC); GomezH@cardiff.ac.u (HLG); MatsuuraM@cardiff.ac.uk (MM)
G54.1+0.3 (Temim et al. 2017; Rho et al. 2018), and also G0.0+0.0 (Lau et al. 2015), G292.0+1.8 (Ghavamian \& Williams 2016), G350.1-0.3 (Lovchinsky et al. 2011), and G357.7+0.3 (Phillips, Ramos-Larios \& Perez-Grana 2009). The majority of SNe with reported high ejecta dust masses to date have been the remnants of core-collapse SNe.

We can now move on from small numbers of individual studies thanks to the availability of large surveys of the Galactic Plane with the Spitzer Space Telescope (hereafter Spitzer; Werner et al. 2004) and the Herschel Space Observatory (hereafter Herschel; Pilbratt et al. 2010); these provide images of hundreds of SNRs from the near- to far-infrared (NIR-FIR; 3.6-500 $\mu \mathrm{m}$ ). Previous works have used areas within these surveys to detect dust signatures associated with SNRs including the Spitzer catalogues of Reach et al. (2006) and Pinheiro Goncalves et al. (2011), and the recent Herschel sample of Chawner et al. (2019, hereafter C19, drawn from the Herschel Infrared Galactic Plane Survey I, Hi-GAL I over $10^{\circ}<|l|<60^{\circ}$ at $\left.70-500 \mu \mathrm{m}\right)$. Those works reported the detection of dust emission associated with SNR structures in 18, 39, and 29 SNRs, respectively, over a region of the Galactic Plane covering $\sim 100 \mathrm{deg}^{2}$, suggesting SN-related dust emission is rather common. 
Prior to the Spitzer and Herschel era, FIR surveys of Galactic SNRs with IRAS by Arendt (1989) and Saken, Fesen \& Shull (1992) detected emission from dust in 11 and 17 SNRs, respectively, within the Galactic Plane $\left(b \leq\left|1^{\circ}\right|\right)$, based on the Green (2014) catalogue. These FIR surveys used observations centred at 12, 25, 60 , and $100 \mu \mathrm{m}$ at respective resolutions of $0.5 \operatorname{arcmin}$ at $12 \mu \mathrm{m}$ and 2 arcmin at $100 \mu \mathrm{m}$. The more recent whole sky survey with AKARI, at wavelengths of $2.4-160 \mu \mathrm{m}$ and angular resolution of 0.5 arcmin in the $65 \mu \mathrm{m}$ band, studied 20 Galactic SNRs (Koo et al. 2016). With the higher resolution of Herschel ( 6 arcsec at $70 \mu \mathrm{m})$ it should be possible to more easily disentangle SNR and unrelated interstellar dust near to the remnant, and to confirm or update the detection levels of these previous studies.

C19 further discovered clear evidence for dust formation in the ejecta of the pulsar wind nebulae (PWNe) G11.2-0.3, G21.5-0.9, and G29.7-0.3 alongside the previously discovered G54.1+0.3, with a detection rate of $\sim 45$ per cent for this class of remnant within the area studied. Such a high detection rate implies that many PWNe may contain dust, some of which can be freshly formed ejecta dust (see also Omand, Kashiyama \& Murase 2019). Though they report that the high detection rates in this SN class may simply be due to observational bias: the dust in the PWNe appears hotter than the surrounding regions and it is easier to disentangle warmer/heated dust structures from unrelated dust in the surrounding cooler ISM.

One remaining critical question is whether or not Type Ia supernovae could also produce dust in their ejecta. Herschel observations of the historical Tycho and Kepler SNRs by Gomez et al. (2012a) showed that although dust structures associated with the SNR material (as traced by X-ray and radio emission) were clearly detected, these dust structures originated from the swept up circumstellar and interstellar medium, respectively (see also Ishihara et al. 2010) and not from freshly formed ejecta dust.

Using Herschel in the FIR to search for dust signatures is not always superior to using MIR observations from, for example, Spitzer (particularly at $24 \mu \mathrm{m}$ where searching for dust signatures can be easier since the hotter dust emission can be more clearly disentangled from interstellar material). However, the increased sensitivity and resolution with Herschel at wavelengths $\geq 70 \mu \mathrm{m}$ compared to lower resolution FIR observatories can be important for disentangling interstellar and SN-related structures in confused regions compared to e.g. Spitzer at the same wavelengths, IRAS, and AKARI (Murakami et al. 2007). The longer Herschel wavebands have the added advantage of being able to reveal the presence of any existing colder dust potentially missed by shorter wavelength observations (e.g. Barlow et al. 2010; Matsuura et al. 2011; Gomez et al. 2012b; Temim et al. 2017; Rho et al. 2018).

Unfortunately, confusion with the ISM is the major limitation when studying dust in SNRs (e.g. in Cas A). Indeed in the Herschel Galactic SNRs studied by Chawner et al. (2019), confusion meant that $\mathrm{SN}$ dust structures could not be separated from unrelated ISM in more than 60 per cent of the sample, and even for those sources with clear FIR dust signatures coincident with known X-ray and radio emission originating from the SNRs, confusion still persists in a large fraction (also noted by Pinheiro Goncalves et al. 2011).

Therefore, in order to verify the importance (or not) of $\mathrm{SNe}$ as dominant dust producers in galaxies and to determine which $\mathrm{SNe}$ form dust in their ejecta material, a larger statistical sample is needed. In this paper we supplement the first-look catalogue from C19 and present a full Herschel Galactic Plane survey of SNRs detected at FIR wavelengths $(\geq 70 \mu \mathrm{m})$. This study covers an area roughly twice as large as that of Chawner et al. (2019), and reveals dusty SNRs that are older than the previous targeted Herschel SNRs such as Cas A and Tycho. In Section 2 we introduce the survey and classify Galactic SNRs with detection levels based on whether there are associated dust features in the Herschel images. Section 3 investigates the properties of the dust for those sources where clear dust features were found. Our results are discussed in Section 4, and our summary is listed in Section 5 .

\section{SURVEY FOR FAR INFRARED SUPERNOVA REMNANT EMISSION}

We study the remaining SNRs in the Galactic Plane (Green 2004) using Hi-Gal (Molinari et al. 2010, 2016) covering $360^{\circ}$ in longitude and $|b| \leq 1$. There are a total of 200 SNRs in this area, although Herschel images for 10 are unavailable, this work therefore includes an additional 119 sources compared to $\mathrm{C} 19$, and an additional 92 and 66 sources compared to the previous dusty catalogues of Galactic SNRs studied using Spitzer in Reach et al. (2006, MIR, $\lambda \leq 24 \mu \mathrm{m})$ and Pinheiro Goncalves et al. $(2011, \lambda \leq 70 \mu \mathrm{m})$ respectively (an additional 163 and 137 SNRs when this work is combined with the Herschel catalogue in C19).

In brief, each remnant was first inspected as a false colour image combining the 70,160 , and $250 \mu \mathrm{m} \mathrm{Herschel} \mathrm{wavebands,}$ regridded and convolved to the resolution of the $250 \mu \mathrm{m}$ band (see the Appendix for the three colour Herschel images, Fig. A1). FIR emission from SNRs can be thermal or non-thermal. Thermal FIR will arise from warm dust such as warm ejecta material, shock-heated dust in the forward or reverse blast waves, photoionized material near a PWN, or swept-up ISM. Non-thermal emission originates from synchrotron emission from shocks or PWNe, although this is not expected to be significant except in the case of plerion objects such as the Crab where synchrotron emission contributes between 19 and 88 percent of the flux in the various Herschel wavebands (De Looze et al. 2019). We compare with multiwaveband images to identify dust emission associated with each SNR which, in principle, should allow us to identify the emission mechanism. For the most part we compare with radio and, where available, X-ray images, both of which trace shock-heated material, see individual notes in Section 2.1 for details. Where radio and X-ray structures originate from a PWN, incident FIR emission may indicate dust in a photoionized region. We can also use X-ray and optical images to identify ejecta material or swept-up/shocked ISM and in some cases, any correlation observed between FIR and MIR or NIR emission can help to identify a variety of FIR sources such as shock-heated dust or swept up ISM.

The method for determining if dust signatures related to $\mathrm{SNe}$ material are present in the Herschel images is described in full in C19 and is the same classification criteria as used in the Spitzer SNR catalogues of Reach et al. (2006) and Pinheiro Goncalves et al. (2011). Following the definition of detection levels from the MIR Spitzer SNR catalogue in Reach et al. (2006, see also C19), we define detections as: $1=$ detection (FIR emission which is clearly correlated with radio, MIR, or X-ray structure and can be distinguished from the ISM), 2 = possible detection (FIR emission in the region of the SNR, potentially related to radio, MIR, or X-ray structure but confused with ISM), 3 = unlikely detection (detection of FIR emission which is probably unrelated to the SNR), and 4 $=$ no detection of FIR emission. We also introduce a new category in this work, ' $i$ ', to account for the fact that in some cases, the only dust signatures associated with the SNR (via radio and/or Xray emission) are found in regions where the shell is known to be interacting with a molecular cloud. Table A1 lists the full 119 Galactic SNRs from Hi-GAL studied in this survey along with the 
Table 1. A summary of the supernova remnants in the Hi-GAL Survey with detection level $=1$ : FIR emission which is clearly correlated with radio, MIR, or X-ray structure and can be distinguished from the ISM.

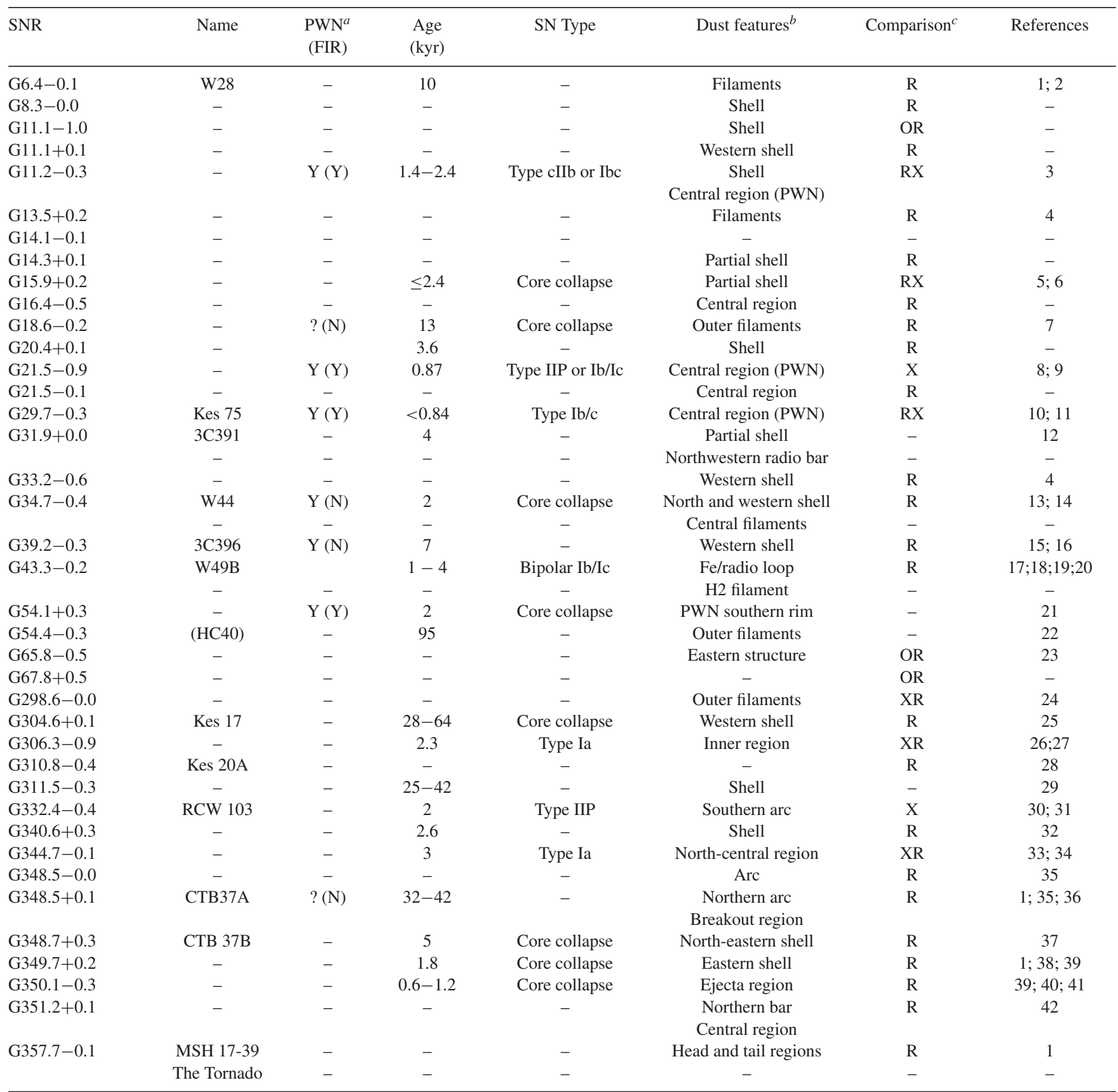

Notes. ' 'Y' indicates that a source contains an associated PWN, '?' indicates an unconfirmed PWN candidate. The FIR detection of the PWN is indicated in the brackets. ${ }^{b}$ Location of FIR detected dust features. ${ }^{c}$ Waveband of previous detection to which FIR structure is compared: $\mathrm{O}=$ optical, $\mathrm{R}=$ radio, $\mathrm{X}=\mathrm{X}$-ray. References for age and SN type for this table are: ${ }^{1}$ Pihlström et al. (2014), ${ }^{2}$ Rho \& Borkowski $(2002),{ }^{3}$ Borkowski, Reynolds \& Roberts $(2016),{ }^{4}$ Case \& Bhattacharya (1998), ${ }^{5}$ Reynolds (2006), ${ }^{6}$ Klochkov et al. (2016), ${ }^{7}$ Voisin et al. (2016), ${ }^{8}$ Bietenholz \& Bartel (2008), ${ }^{9}$ Bocchino et al. (2005), ${ }^{10}$ Leahy, Tian \& Wang (2008), ${ }^{11}$ Morton et al. (2007), ${ }^{12}$ Chen et al. (2004), ${ }^{13}$ Wolszczan, Cordes \& Dewey (1991), ${ }^{14}$ Zhu et al. (2013), ${ }^{15}$ Harrus \& Slane (1999), ${ }^{16}$ Leahy \& Ranasinghe (2016), ${ }^{17}$ Pye et al. (1984), ${ }^{18}$ Smith et al. (1985), ${ }^{19}$ Hwang, Petre \& Hughes (2000), ${ }^{20}$ Lopez et al. (2013), ${ }^{21}$ Bocchino, Bandiera \& Gelfand (2010), ${ }^{22}$ Park et al. (2013), ${ }^{23}$ Shan et al. (2018), ${ }^{24} \mathrm{Bamba}$ et al. (2016), ${ }^{25} \mathrm{Combi}$ et al. (2010b), ${ }^{26}$ Reynolds et al. (2013), ${ }^{27} \mathrm{Combi}$ et al. (2016), ${ }^{28}$ Andersen et al. (2011), ${ }^{29}$ Pannuti et al. (2014a), ${ }^{30}$ Nugent et al. (1984), ${ }^{31}$ Frank, Burrows \& Park (2015), ${ }^{32}$ Caswell et al. (1983b), ${ }^{33}$ Giacani et al. (2011), ${ }^{34}$ Yamaguchi et al. (2012), ${ }^{35}$ Pannuti et al. (2014a), ${ }^{36}$ Tian \& Leahy (2012), ${ }^{37}$ HESS Collaboration (2008b), ${ }^{38}$ Tian \& Leahy (2014), ${ }^{39}$ Yasumi et al. (2014), ${ }^{40}$ Gaensler et al. (2008), ${ }^{41}$ Lovchinsky et al. (2011), ${ }^{42}$ Dubner et al. (1993).

71 sources studied by $\mathrm{C} 19$, with the 39 detected level 1 SNRs summarized in Table 1. Individual details provided for each SNR assigned a detection rate of 1 or 2 in this work ( 10 level 1 and 11 level 2) are listed in the following Section. We note that the availability and quality of ancillary data and the depth of those observations is not the same for all SNRs, which makes it impossible to automate the classification of the detection levels, and thus requires manual intervention and interpretation. This could introduce a bias towards 

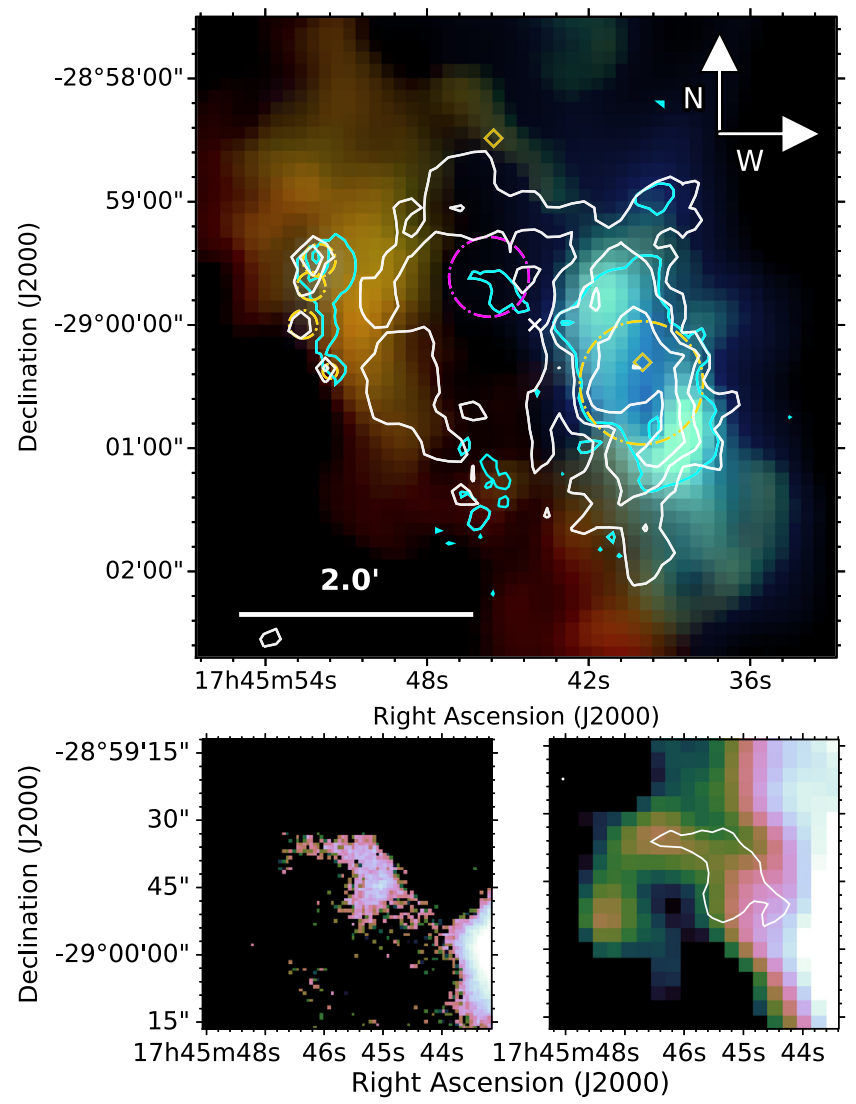

Figure 1. G0.0+0.0, Sgr A East - top: Herschel three colour image with JVLA $5.5 \mathrm{GHz}$ contours overlaid in white and SOFIA $37.1 \mu \mathrm{m}$ contours overlaid in cyan, colours are red $=250 \mu \mathrm{m}$, green $=160 \mu \mathrm{m}$, and blue $=70 \mu \mathrm{m}$. Bottom: A zoom in on the dust factory detected within the magenta circle (top) by Lau et al. (2015). left-hand panel: the SOFIA $37.1 \mu \mathrm{m}$ detection from their work, and right-hand panel: the Herschel $70 \mu \mathrm{m}$ image with SOFIA $37.1 \mu \mathrm{m}$ contours overlaid. Here the Herschel and SOFIA data have been scaled considerably to show this faint feature. (For the bottom panels we use the cube helix colour scheme (Green 2011).) The gold circles to the east indicate the locations of $\mathrm{H}$ II regions (Goss et al. 1985) and the gold circle to the west indicates the location of Sgr A West. The diamond to the north indicates the location of the associated neutron star, 'The Cannonball', and the diamond to the west indicates the location of Sgr A*. The white cross shows the radio coordinates of the SNR centre from Green (2014). The same Herschel colour combinations and orientation are used in Figs 2-19.

bright, well-studied sources that we classify as detections here, similarly this could also be a factor in the previously quoted rates for SNRs detected in near-MIR catalogues with Spitzer (Reach et al. 2006; Pinheiro Goncalves et al. 2011).

\subsection{Results for individual remnants}

Notes on individual sources classed as detection level 1 (FIR emission which is clearly correlated with radio, MIR, or X-ray SNR structure and can be distinguished from the ISM) and detection level 2 (FIR emission in the region of the SNR, potentially related to radio, MIR, or X-ray structure but confused with ISM) are provided in this section.

G0.0 + 0.0 (Sgr A East): This CCSN remnant (Fig. 1) has an elliptical radio shell with a plume of radio and X-ray emission at the northern edge. There is an associated runaway neutron star, the Cannonball, and a PWN in front of this plume (e.g. Nynka et al.
2013; Zhao, Morris \& Goss 2013). There are numerous unrelated $\mathrm{H}$ II regions in this complex; the largest, Sgr A West, is observed within the SNR shell on the western side and has a spiral structure. Four smaller regions are found to the east, just outside of the SNR radio shell. Observations of $327 \mathrm{MHz}$ absorption place Sgr A West in front of the SNR (Yusef-Zadeh \& Morris 1987), although their relative distances are uncertain. There is some evidence that the two sources are at a similar distance and there has been some interaction between the expanding shell and Sgr A West (Yusef-Zadeh \& Goss 1999; Yusef-Zadeh, Melia \& Wardle 2000; Maeda et al. 2002), although this is controversial.

The SNR age and X-ray observations of hot ejecta at its centre both suggest that the reverse shock has already reached the centre of this source (Maeda et al. 2002). Lau et al. (2015) completed a detailed analysis of the central region using data in the range 5.8$70 \mu \mathrm{m}$ from Spitzer IRAC, the Faint Object Infrared Camera for the SOFIA Telescope (FORCAST), and Herschel PACS $(70 \mu \mathrm{m})$. These observations indicate the presence of dust within the SNR central region, north-east of Sgr A West (indicated by the magenta circle in the top panel of Fig. 1). They studied the 5.8-37.1 $\mu \mathrm{m}$ emission from several regions in and around the location of the SNR dust, using the $70 \mu \mathrm{m}$ flux as an upper limit. They assumed it is composed of amorphous carbon grains with two grain sizes (large grains of $\sim 0.04 \mu \mathrm{m}$ and very small grains of $\sim 0.001 \mu \mathrm{m}$ ) which is heated by a nearby cluster of massive young stars. This gave a total ejecta mass of $\sim 0.02 M_{\odot}$ of warm $(\sim 100 \mathrm{~K})$ dust which has survived the passage of the reverse shock.

We compare Herschel maps with the JVLA $5.5 \mathrm{GHz}$ image (Zhao et al. 2013). We reduced the VLA data, using the Common Astronomy Software Applications (CASA) of the National Radio Astronomy Observatory (NRAO). The $C$-band (4.5-6.4 GHz) continuum image of Sgr $\mathrm{A}^{*}$ was produced by combining multiple VLA observations taken between 2012 March and July. The data were processed in the AIPS software package (Greisen 2003), following standard calibration and wide-band imaging procedures.

Using the five bands of Herschel data from Hi-GAL we search for dust features associated with the SNR and with the Lau et al. (2015) dust discovery (see the bottom panel of Fig. 1). We find that the entire region inside the SNR shell is extremely confused in the Herschel bands (as originally noted by Lau et al. 2015). The flux at $70 \mu \mathrm{m}$ due to background emission (determined by placing multiple apertures across the map outside of the bright dust features) is of the order of $1000 \mathrm{Jy}$. Integrating the emission within the Lau et al. (2015) dust region (cyan contours in Fig. 1) and subtracting the background we estimate that $\sim 95$ per cent of the flux in that area originates from unrelated background flux i.e. there is no significant detection above the background noise in the Herschel 70-500 $\mu \mathrm{m}$ bands. The $3 \sigma$ upper limit we derive for the dust region from the $70 \mu \mathrm{m}$ map is $\sim 12 \mathrm{Jy}$ which is consistent with the Lau et al. (2015) estimate. This region is too heavily confused to constrain if there is cooler dust at this location using the Herschel images and therefore we assign this a detection level 2 .

There is bright emission to the west in the 70-250 $\mu \mathrm{m}$ bands at the location of the $\mathrm{H}$ II region Sgr A West which appears bluer compared to the surrounding dust emission (Fig. 1), and which is coincident with the brightest region in the radio. There is also an IR structure to the east of the source in all Herschel wavebands (orange structure in the top panel Fig. 1). It is thought that the SNR is interacting with a molecular cloud on this side (e.g. Sjouwerman, Pihlström \& Fish 2010; Tsuboi, Miyazaki \& Uehara 2015). This continuum emission may originate from shock-heated dust, however, it does 


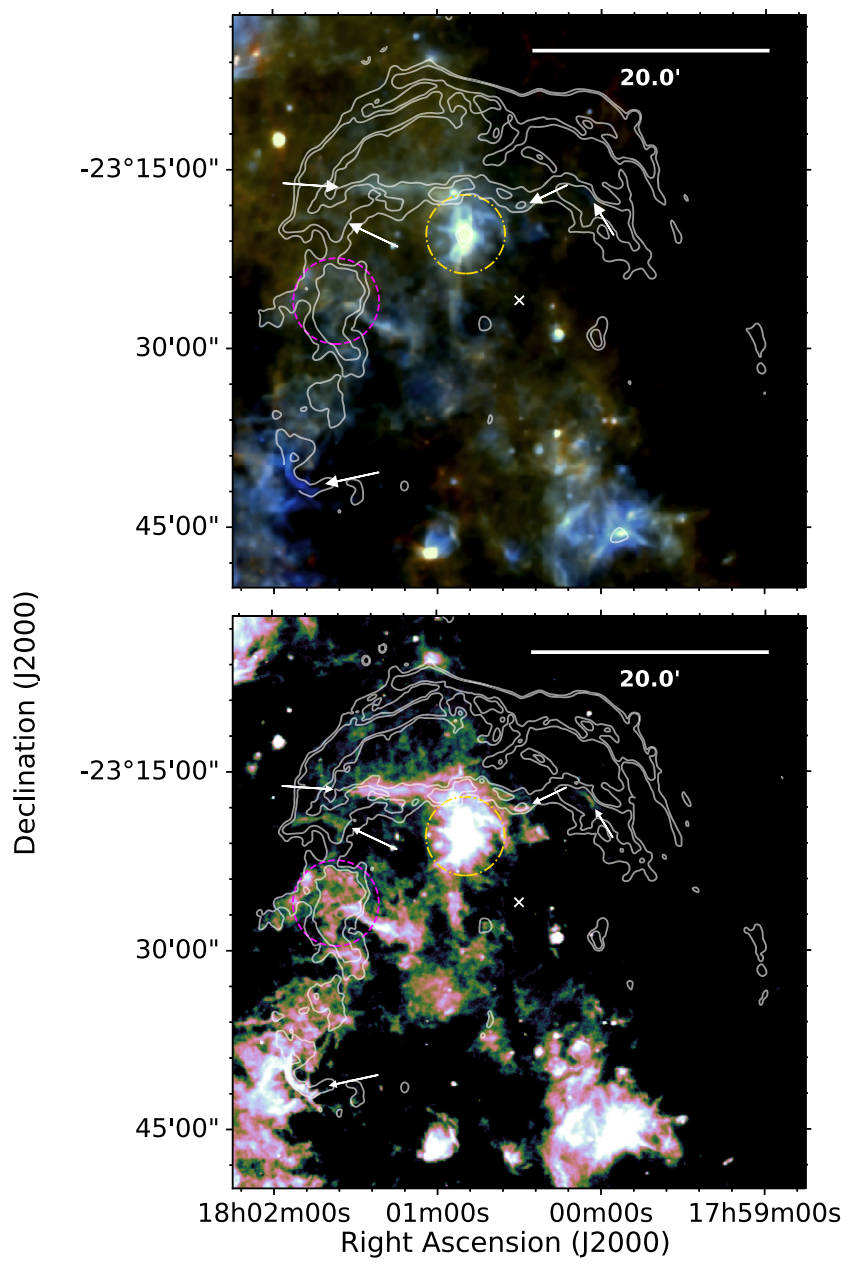

Figure 2. G6.4-0.1, W28 - Top: Herschel three colour image and bottom: Herschel $70 \mu \mathrm{m}$ image, both with radio $(90 \mathrm{~cm})$ contours overlaid. The arrows and magenta circle indicate filaments of $70 \mu \mathrm{m}$ emission which are coincident with radio structure. There is FIR emission at the location of other radio emission, although the structure is different and association is unclear. The gold circle indicates the location of an unrelated $\mathrm{H}$ II region. The white cross shows the radio coordinates of the SNR centre from Green (2014).

not correspond to the radio morphology and it is more likely that it is unrelated.

G6.4-0.1 (W28): W28 is a well-studied mixed morphology remnant with evidence of a molecular cloud-SN blast wave interaction (e.g. Wootten 1981; Reach et al. 2005). We see many filaments of $70 \mu \mathrm{m}$ emission which follow the radio contours in Fig. 2. Although the proximity of $\mathrm{H}$ II regions makes this region confused, we propose that the structure in the Herschel data (arrows and magenta circle in Fig. 2) on the eastern side of the remnant, and to the north is associated with the SNR due to its close correlation with radio emission. The $70 \mu \mathrm{m}$ emission on the eastern side is also coincident with shocked $\mathrm{H}_{2}$ emission (Reach et al. 2005) from the interaction border between the SNR and molecular clouds. Furthermore, the dusty region in the magenta circle and the northernmost part of the dust feature indicated by the arrows overlap with shocked molecular gas $\left({ }^{12} \mathrm{CO}(3-2)\right.$, fig. 1(b) in Zhou et al. 2014, see also Arikawa et al. 1999). Although the region is confused in the Herschel images, given the strong association of the $70 \mu \mathrm{m}$ filaments with the radio structures, we classify this as a level 1 detection.

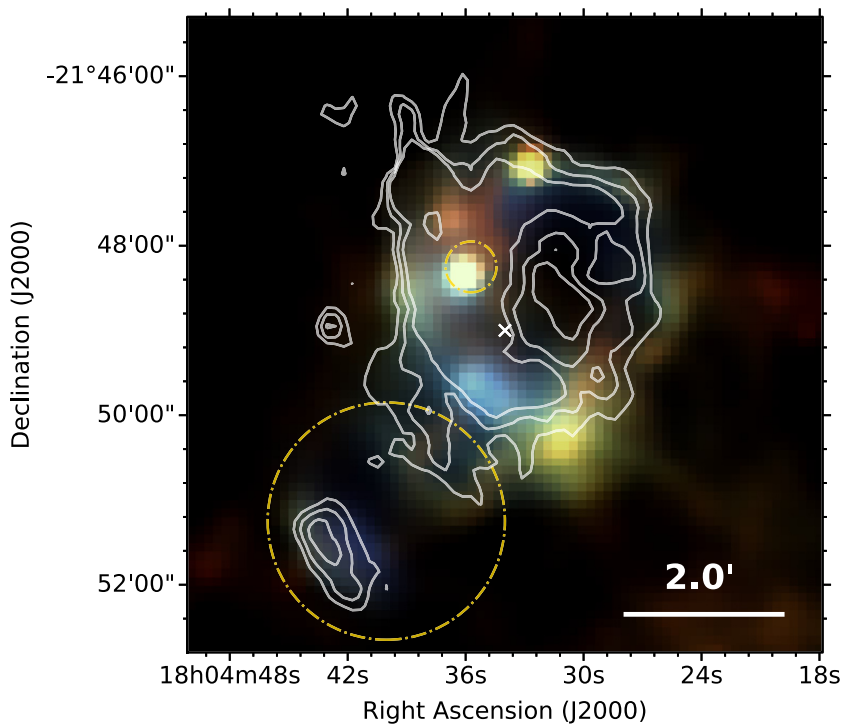

Figure 3. G8.3-0.0 - Herschel three colour image with VLA $20 \mathrm{~cm}$ contours overlaid. A shell structure is detected across all Herschel wavebands. There is some variation in the FIR structure compared with the radio, however there is coincidence between the two. The large gold circle indicates the location of an IR bubble. The white crosses shows the X-ray coordinates of the SNR centre and the small gold circle indicates the location of a maser.

G8.3-0.0: Brogan et al. (2006) detected a radio shell at $90 \mathrm{~cm}$ with a very bright region at $\alpha=18^{\mathrm{h}} 04^{\mathrm{m}} 38^{\mathrm{s}}, \delta=-21^{\circ} 47^{\prime} 17^{\prime \prime}$. There is a shell of emission detected across all Herschel wavebands which correlates with the radio structure as shown in Fig. 3. As such, this satisfies our criteria for a level 1 detection. There is a marked colour variation within this shell. At wavelengths longer than $70 \mu \mathrm{m}$ there is a bright patch to the south-west, and emission is detected at all wavelengths from the location of a maser at $\alpha=18^{\mathrm{h}} 04^{\mathrm{m}} 36.02^{\mathrm{s}}$, $\delta=-21^{\circ} 48^{\prime} 19.6^{\prime \prime}$ (Hewitt \& Yusef-Zadeh 2009; Green et al. 2010). There is also FIR emission across all Herschel wavebands to the south-east of the SNR, near $\alpha=18^{\mathrm{h}} 04^{\mathrm{m}} 43.5^{\mathrm{s}}, \delta=-21^{\circ} 51^{\prime} 29.5^{\prime \prime}$, although this region is at the edge of a FIR bubble (Simpson et al. 2012, Fig. 3) making its association with the SNR unclear.

G9.9-0.8: This SNR has a shell structure detected in radio (90 cm; Brogan et al. 2006) and optical (H $\alpha$; Stupar \& Parker 2011) wavebands. As seen in Fig. 4, there is FIR emission detected at the location of the western edge of the radio shell. However, there are multiple H II regions and FIR bubbles (Simpson et al. 2012) at this location, and the FIR structure is distributed differently to that of the radio. Although molecular hydrogen from an interaction between the SNR and molecular clouds was discovered in Hewitt \& Yusef-Zadeh (2009), there is no accompanying image to compare with the dust features. We therefore argue that the FIR features are potentially related to the SNR, as such this satisfies our criteria for a level 2 detection.

G12.7-0.0: Brogan et al. (2006) detected a radio shell from this SNR. There is FIR emission in Fig. 5 coincident with the southwestern region of the radio shell, although there is a FIR bubble (Simpson et al. 2012) at this location and therefore we cannot be sure of the origin of this emission. We therefore classify this as a level 2 detection.

G13.5+0.2: We detect filaments of emission at 70 and $160 \mu \mathrm{m}$ which correlate with VLA $20 \mathrm{~cm}$ radio structure (Helfand et al. 1989) as indicated by the arrows in Fig. 6 . There is $70 \mu \mathrm{m}$ emission along the south-western edge, within the VLA contours. It is thought 


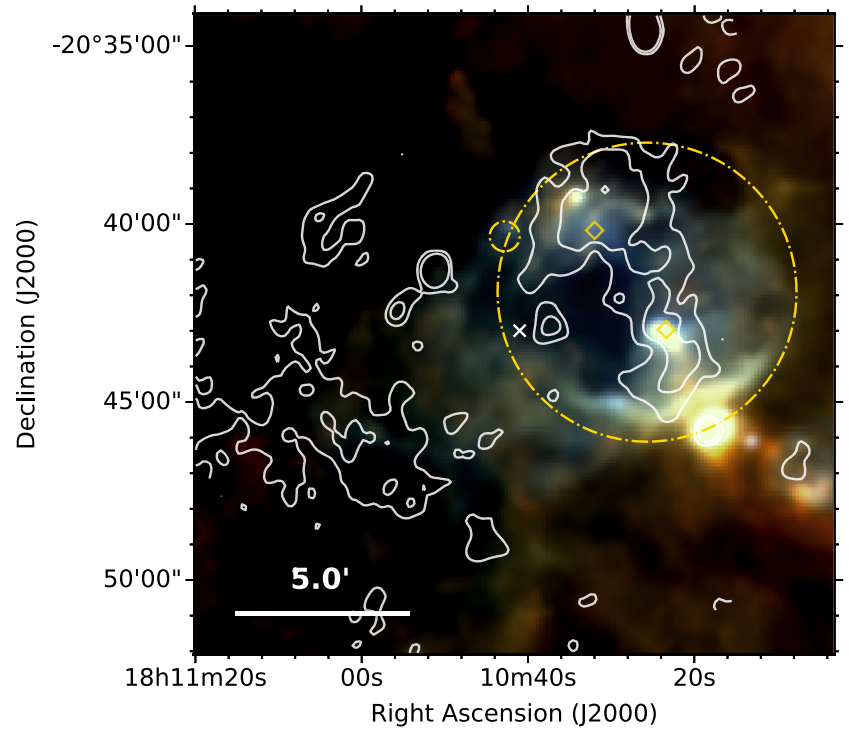

Figure 4. G9.9-0.8 - Herschel three colour image with VLA $90 \mathrm{~cm}$ contours overlaid. There is FIR emission in a shell structure at the western/northwestern edge of the radio shell. However, the morphology of the structures are different and there are multiple $\mathrm{H}$ II regions (gold diamonds) and FIR bubbles (gold circles) (Simpson et al. 2012), as such, we cannot confirm if this is associated with the $\mathrm{SN}$. The white crosses shows the radio coordinates of the SNR centre.

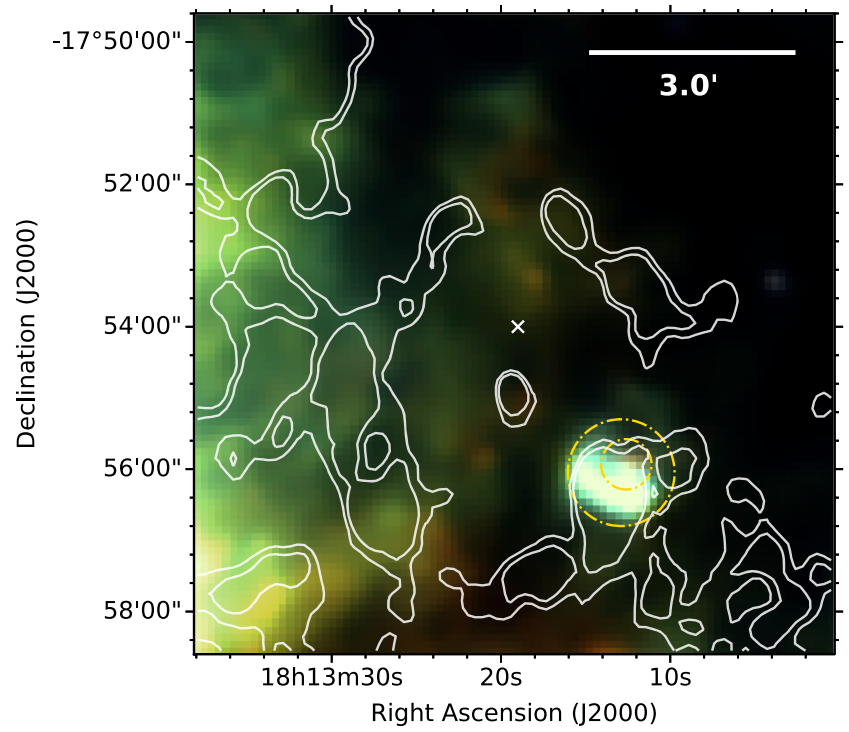

Figure 5. G12.7-0.0 - Herschel three colour image with VLA $20 \mathrm{~cm}$ contours overlaid. The region is very confused to the east, there is FIR emission in the south-western region of the SNR shell. There are also FIR bubbles at this location (Simpson et al. 2012), as indicated by the gold circles. The white crosses shows the radio coordinates of the SNR centre.

that this may be a ridge of shock-heated dust resulting from the interaction between the SNR and surrounding molecular clouds, which is in agreement with the detection of $\mathrm{H}_{2}$ from this source (Froebrich et al. 2015). There is more confusion in the longer wavebands and there does not seem to be any associated structure in those bands. Based on the coincidence with the $70 \mu \mathrm{m}$ filaments and radio features, we therefore classify this as detection level 1.

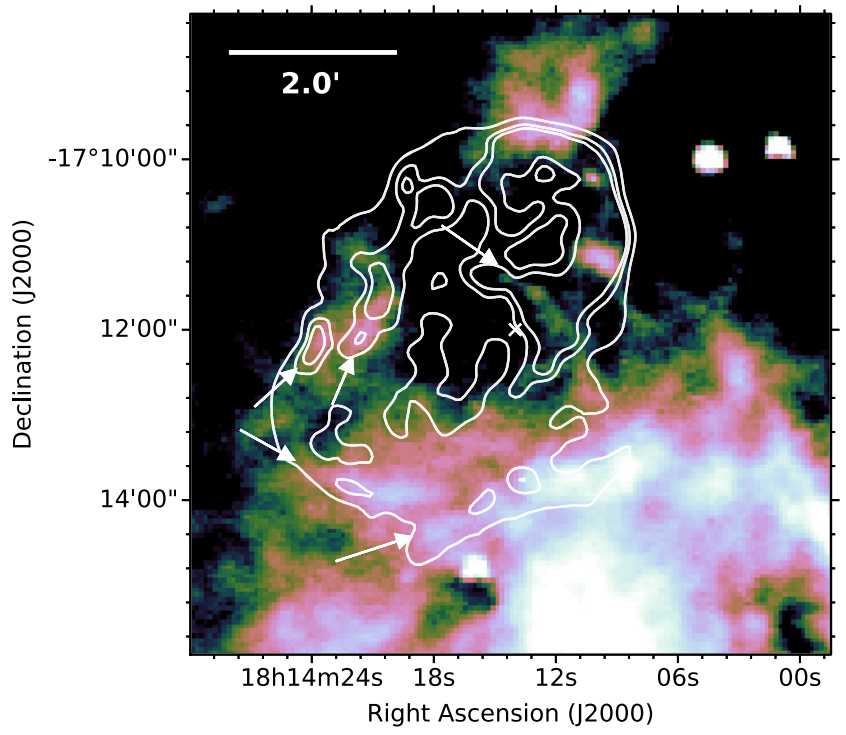

Figure 6. G13.5+0.2 - Herschel $70 \mu \mathrm{m}$ image with VLA $20 \mathrm{~cm}$ contours overlaid. Filaments are detected at $70 \mu \mathrm{m}$ which are coincident with radio emission, and are indicated by the arrows. These are more confused at $160 \mu \mathrm{m}$, and cannot be distinguished from surrounding ISM in the longer Herschel wavebands. The white cross shows the radio coordinates of the SNR centre from Green (2014).

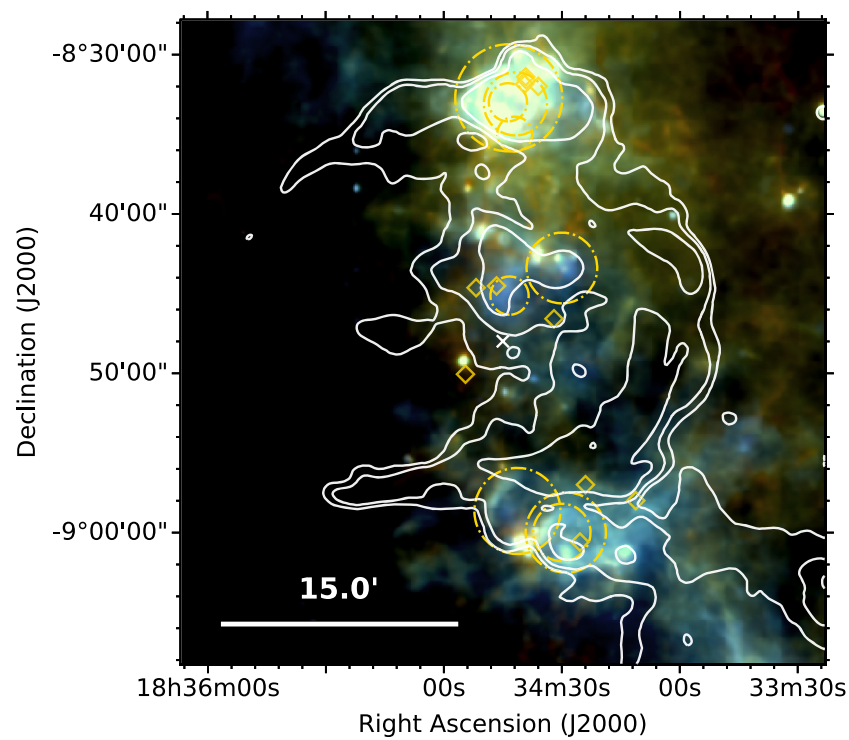

Figure 7. G23.3-0.3 (W41) - Herschel three colour image with VGPS $21 \mathrm{~cm}$ contours overlaid. The gold circles indicate the locations of some of the unrelated sources in this region, such as molecular clouds and bubbles. The gold diamonds at $\alpha=18^{\mathrm{h}} 34^{\mathrm{m}} 51.8^{\mathrm{s}}, \delta=-08^{\circ} 44^{\prime} 38.2^{\prime \prime}$ and $\alpha=$ $18^{\mathrm{h}} 34^{\mathrm{m}} 46.6^{\mathrm{s}}, \delta=-08^{\circ} 44^{\prime} 31.4^{\prime \prime}$ indicate the locations of a gamma-ray source (HESS J1834-087) and OH (1720 MHz) maser lines. The other gold diamonds indicate the locations of masers.

G23.3-0.3 (W41): This SNR is in a very crowded region of the Galactic Plane, where there are multiple dense cores, YSOs, $\mathrm{H}$ II regions, and bubbles. The presence of a gamma-ray source (HESS J1834-087) and OH (1720 MHz) maser lines near the centre of the source (indicated in Fig. 7) suggest that the SNR may be interacting with a molecular cloud (HESS Collaboration 2015). Several molecular clouds are labelled in Fig. 7 with gold 


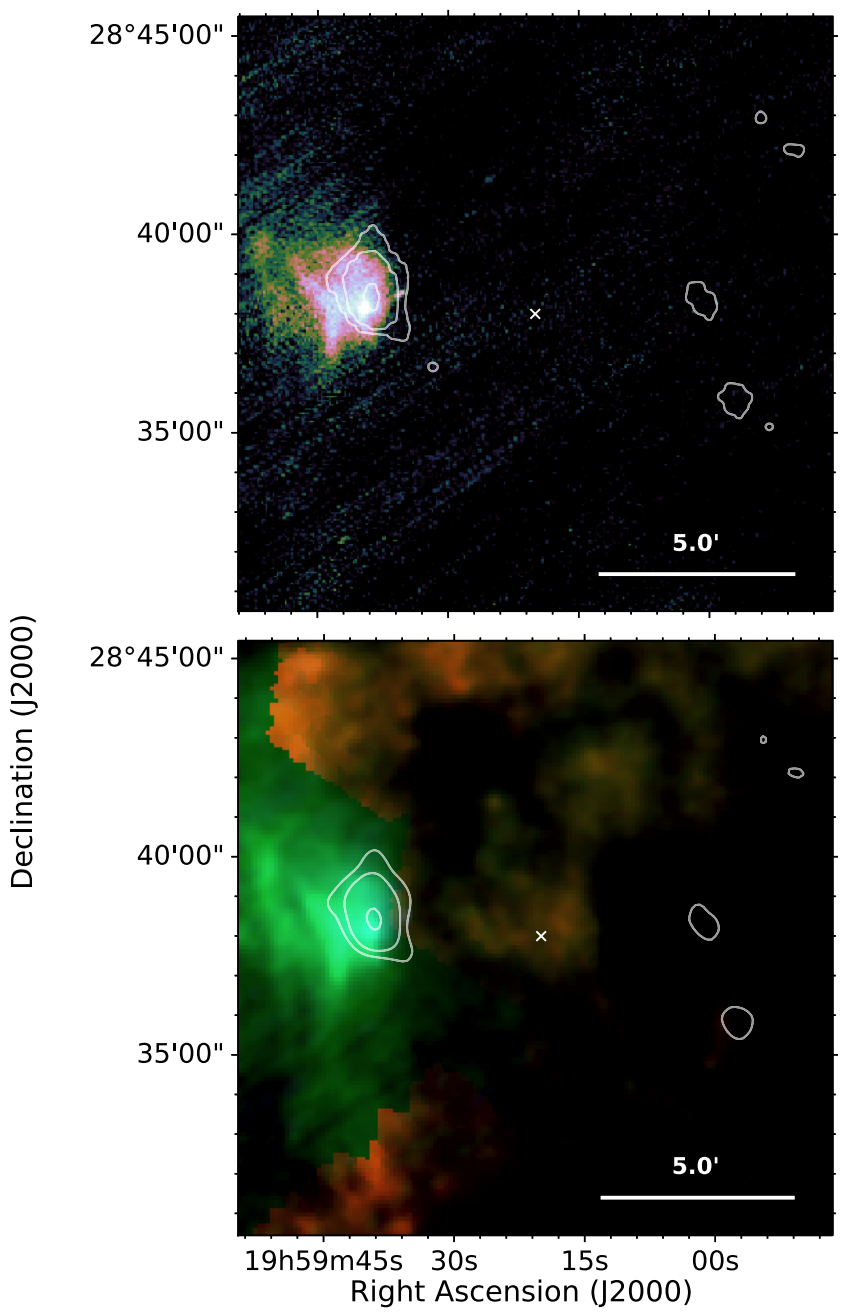

Figure 8. G65.8-0.5 - Top: Herschel $70 \mu \mathrm{m}$ image and bottom: Herschel three colour image, both with radio NVSS $1.4 \mathrm{GHz}$ contours overlaid. FIR structure is detected at the eastern edge of the SNR, at the location of radio and $\mathrm{H} \alpha$ structure (Sabin et al. 2013). An unidentified region of FIR emission is detected at the centre of the source at $160-500 \mu \mathrm{m}$. The white cross shows the radio coordinates of the SNR centre from Green (2014).

circles. There is bright FIR emission at the location of the radio structure, however the clearest FIR is also coincident with molecular clouds. Although there may be SNR-related dust in this region, we cannot determine its association because of confusion, this therefore satisfies our criteria for a level 2 detection.

G65.8-0.5: This object was only recently classified as a SNR by Sabin et al. (2013) who used $\mathrm{H} \alpha$ to identify its nature. We compare $\mathrm{H} \alpha$ and NVSS images with Herschel, finding bright 70 and $160 \mu \mathrm{m}$ emission at the east of the source at the location of the Sabin et al. (2013) $\mathrm{H} \alpha$ structure. We propose that this 70 and $160 \mu \mathrm{m}$ feature is associated with the SNR, although the structure is offset to, and more extended than the radio emission seen in the NVSS $1.4 \mathrm{GHz}$ image (Fig. 8). Unfortunately the Herschel SPIRE maps at 250-500 $\mu \mathrm{m}$ do not cover the area where the $\mathrm{H} \alpha$ and radio structure is seen. Additionally, at $160-500 \mu \mathrm{m}$ there is a region of emission detected at the radio centre of the SNR, although this does not correlate with radio emission and we cannot determine if this is associated. Given the close spatial coincidence between the Herschel emission, $\mathrm{H} \alpha$ and radio structures to the east of the SNR, we classify this as a level 1 detection, though we note that here we do not have the full colour information in the Herschel bands.

G67.8+0.5: Like G65.8-0.5, this source was only recently identified as a SNR by Sabin et al. (2013). There is bright $70 \mu \mathrm{m}$ emission at $\alpha=19^{\mathrm{h}} 59^{\mathrm{m}} 57.5^{\mathrm{s}}, \delta=30^{\circ} 51^{\prime} 26^{\prime \prime}$ (Fig. 9.), coinciding with the location of an unidentified IRAS source (IRAS $19579+3043$ ) and with emission in the WISE $22 \mu \mathrm{m}$ archive image (though at lower resolution than Herschel $70 \mu \mathrm{m}$ ). Here, the FIR is anticorrelated with the radio as the Herschel FIR peak is located in a gap in the radio structure. There is also brighter $70 \mu \mathrm{m}$ emission than the surroundings (bluer FIR than the surrounding emission) indicating the presence of warmer dust emission. Although this does not align with the detection criteria defined in Section 2, we expect that this emission is associated with the SNR due to the FIR colours and the coincidence with the radio centre. There is also a bright shell across all Herschel wavebands to the west and south-western edge of the SNR. We propose that the central source and outer shell are associated with the SNR and therefore classify this SNR as a level 1 detection. It is possible that the central emission, which lacks radio emission, could be cold SN ejecta dust (as seen in Cas A; Barlow et al. 2010), while the shell emission is from the ISM. Further follow up observations in other wavelengths would be needed to investigate this. The temperature map of this SNR is discussed in Fig. 21.

G298.6-0.0: This is a mixed-morphology SNR, with an X-ray filled centre and radio shell (Rho \& Petre 1998; Bamba et al. 2016). A filament of dust is detected in all Herschel wavebands (see Fig. 10.) at the eastern edge of this source at the location of a bright radio filament detected by MOST (Molonglo Observatory Synthesis Telescope) at $843 \mathrm{MHz}$ (Whiteoak \& Green 1996). There is also dust detected along the south of the radio structure. At $70 \mu \mathrm{m}$ this extends along the length of the radio contours and is brightest at the western edge, where emission at the other Herschel wavebands is found. We therefore classify this as a level 1 detection. Comparing the Suzaku X-ray image from Bamba et al. (2016), these features are not correlated with the centrally filled X-ray emission, and therefore are likely to originate from swept up interstellar material given the coincidence with the edge of the radio shell.

G306.3-0.9: This source is a recently discovered remnant of a Type Ia SN (Reynolds et al. 2013) in which the reverse shock has heated the central ejecta region (Combi et al. 2016). The three colour Herschel image is displayed in the top panel of Fig. 11. The overall morphology in the X-ray (Reynolds et al. 2013; Combi et al. 2016) is a semicircular shape with strong radio and X-ray emission in the southwest, suggestive of an interaction with an interstellar cloud or an asymmetric SN explosion. Both X-ray and radio emission are fainter in the north. Combi et al. (2016) showed that the SNR is brighter in softer X-rays towards the centre and found distinct shock-heated ejecta regions in the northeast, central and southwestern areas (see their fig. 7) with enhanced abundances and temperatures. Similarly, they saw distinct X-ray regions in the north-west and south of the SNR that they attributed to shockfronts interacting with the interstellar medium.

Comparisons with the X-ray, radio, and hot dust emitting at $24 \mu \mathrm{m}$ using Spitzer observations were presented in Reynolds et al. (2013) and Combi et al. (2016). In the latter study, they noted that the $24 \mu \mathrm{m}$ morphology from shocked dust grains is shell-like, matching the distribution observed in hard X-rays. The former study noted that an additional ridge feature to the south seen in radio and $\mathrm{X}$ ray images is also correlated with the $24 \mu \mathrm{m}$ emission, commenting that this could originate from swept up dust or ejecta dust. Reynolds et al. (2013) also mention that Herschel 160-500 $\mu \mathrm{m}$ images show 


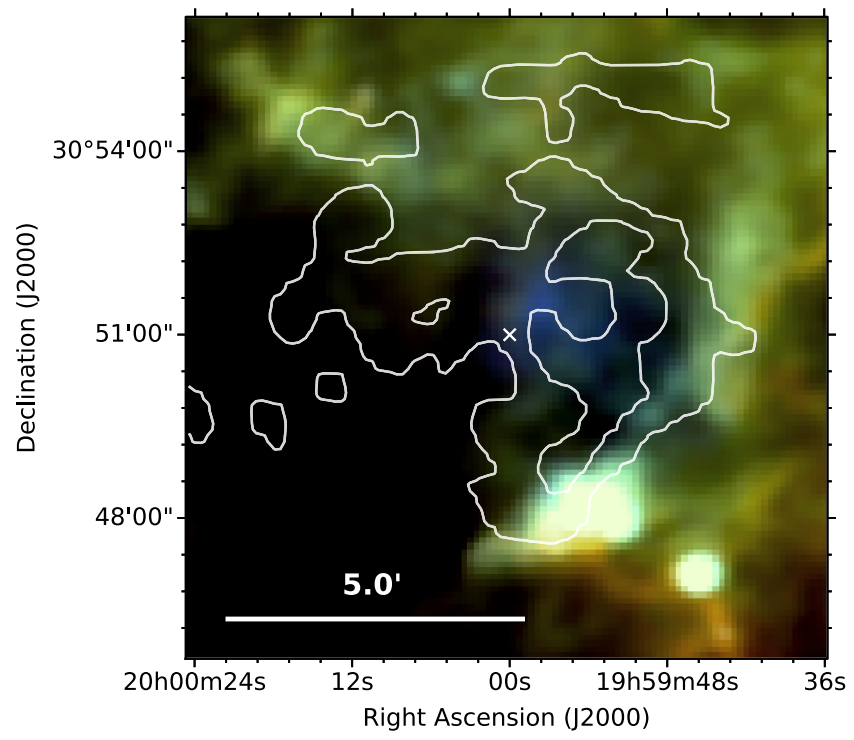

Figure 9. G67.8+0.5 - Herschel three colour image with NVSS $1.4 \mathrm{GHz}$ contours overlaid. Dust emission is detected at $70 \mu \mathrm{m}$ from a faint radio source, and at all Herschel wavelengths from a partial shell along the western and south-western edges of the SNR. The white cross shows the radio coordinates of the SNR centre from Green (2014).

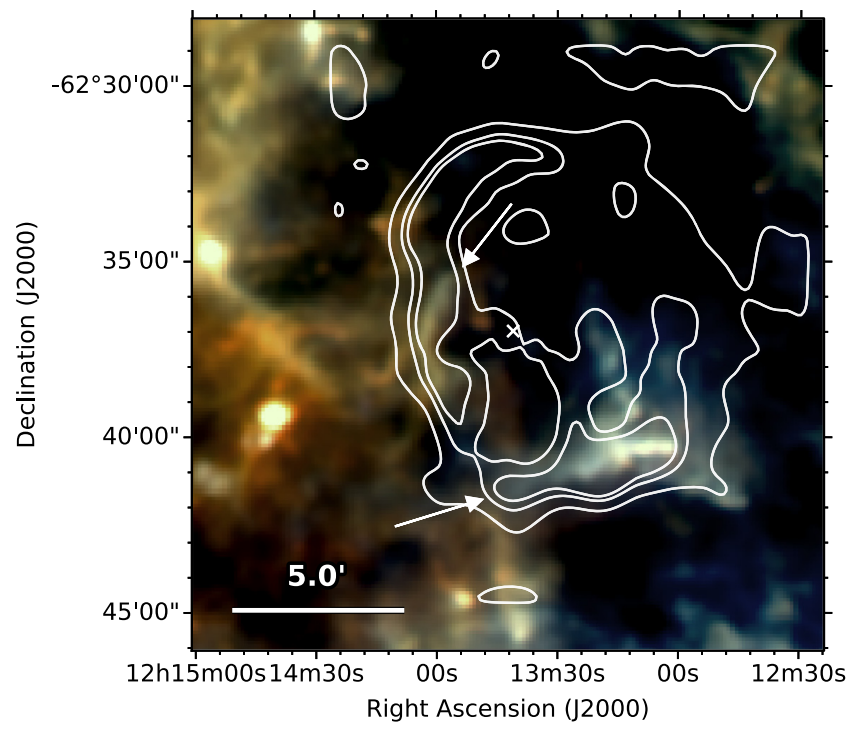

Figure 10. G298.6-0.0 - Herschel three colour image with $843 \mathrm{MHz}$ (Molonglo Observatory Synthesis Telescope, MOST) contours overlaid. Dust is detected in filaments at the east of the source, and in a ridge along the southern edge as indicated by the arrows. The white cross shows the radio coordinates of the SNR centre from Green (2014).

an overdensity of dust in the north where the X-ray emission is the faintest.

In the Herschel image, the dust emission is concentrated in the south, with very little dust at $70 \mu \mathrm{m}$ in the north-east. The Herschel dust features that are bright at $70 \mu \mathrm{m}$ (blue clumps in Fig. 11) overlap with the known $24 \mu \mathrm{m}$ features, making this a clear level 1 detection. The bottom panel of Fig. 11 compares the Herschel emission at $70 \mu \mathrm{m}$ with the distinct X-ray regions observed by Combi et al. (2016). NE, C, and SW show the location of the reverse shock heated ejecta regions and NW and $\mathrm{S}$ show regions
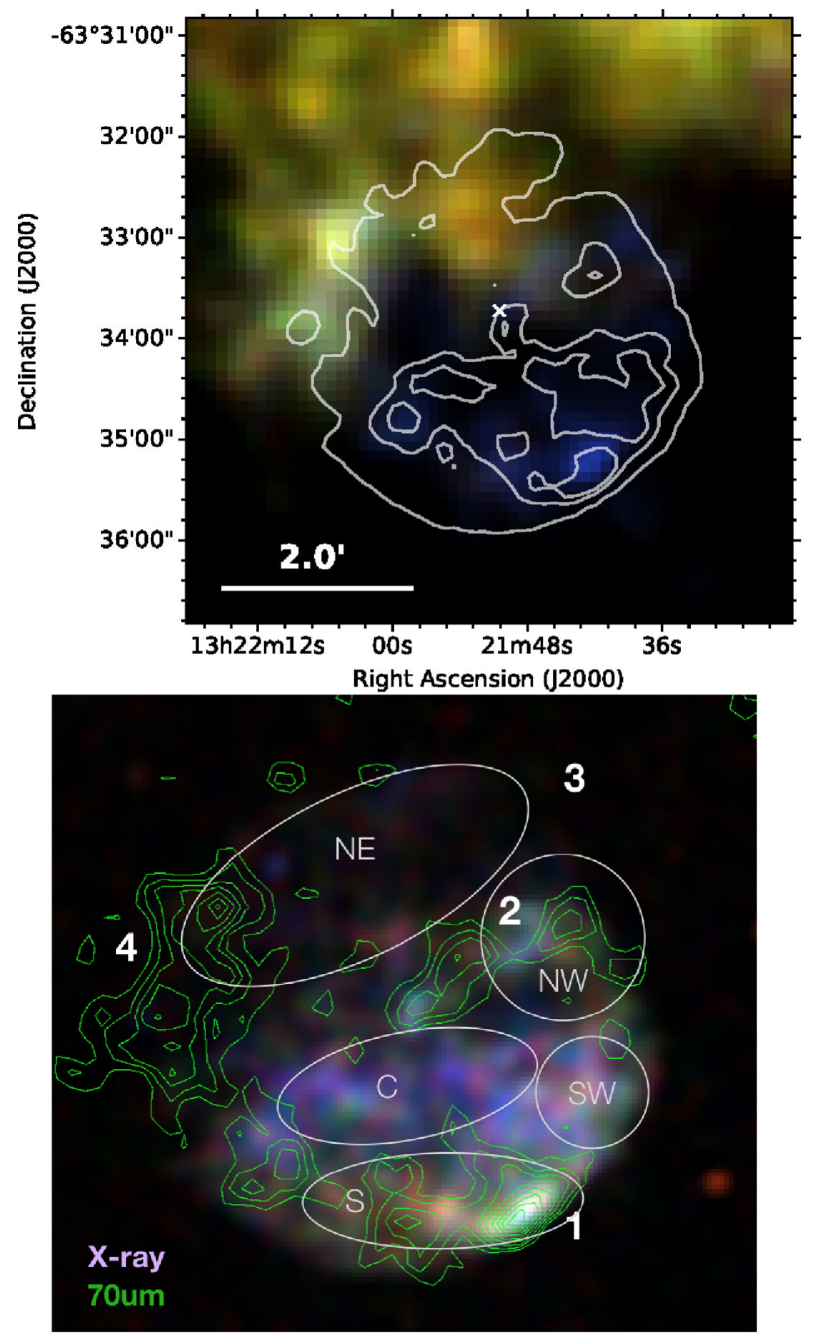

Figure 11. G306.3-0.9 - Top: Herschel three colour image with Chandra contours overlaid. Dust emission is detected at $70 \mu \mathrm{m}$ inside the X-ray contours, especially towards the south-west where there is the brightest $\mathrm{X}$-ray emission. The white cross shows the X-ray coordinates of the SNR centre. Bottom: three colour X-ray image using Chandra archive images at $0.73-1.47 \mathrm{keV}$ (red), $1.47-2.2 \mathrm{keV}$ (green), and $2.2-3.3 \mathrm{keV}$ (blue) with Herschel $70 \mu \mathrm{m}$ contours overlaid. See Reynolds et al. (2013), Combi et al. (2016) for further details of the X-ray features. X-ray 'distinct' regions from Combi et al. (2016) are labelled, where regions NE, C, and SW are attributed to ejecta material, and regions NW and S are consistent with foreground shock material. Dust features seen in the Herschel images are labelled 1-4.

where the X-ray originates from forward shock material. We see a new dust feature in the Herschel maps (labelled '4' in Fig. 11), to the east of the centre of the SNR, which lies slightly ahead of the radio shell. This is a bright elongated structure to the east of the SNR which has two peaks, and lies slightly ahead of the radio shell. It appears to overlap with the eastern-most part of the NE X-ray region from Combi et al. (2016) but has very little associated $\mathrm{X}$-ray and $24 \mu \mathrm{m}$ emission. This dust feature has a very different colour to features 1 and 2 , and is similar to feature 3 , suggesting the dust temperature is cooler. Given the lack of X-ray and radio emission associated with features 3 and 4 and their different colour, we suggest they are unrelated to the SNR even though feature 4, at first glance, appears to be a continuation of the southern dust shell along the eastern side. Instead we propose these are part of a dust 


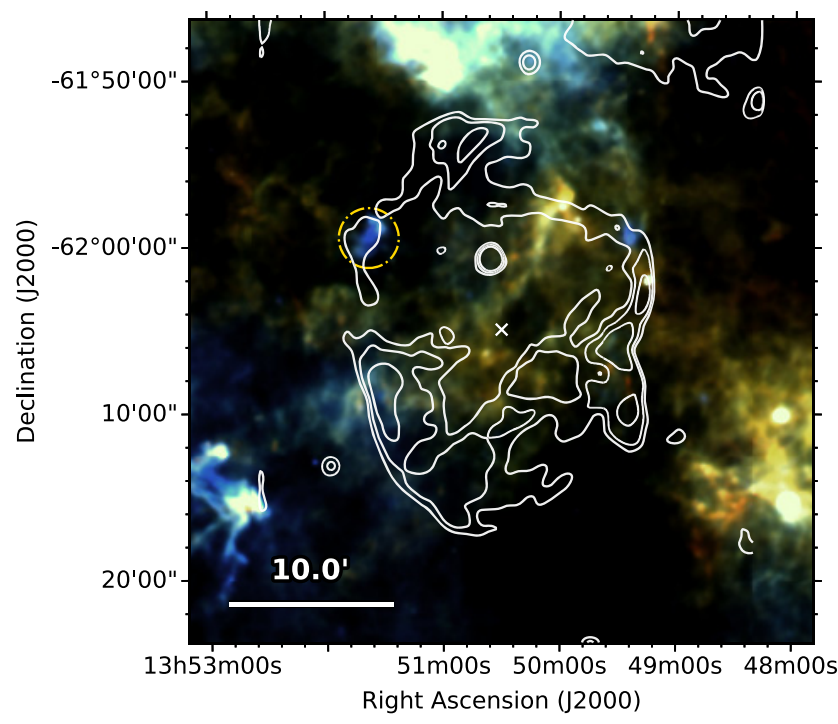

Figure 12. G309.8+0.0 - Herschel three colour image with Chandra contours overlaid. The gold circle indicates a bright knot of $70 \mu \mathrm{m}$ emission which may be associated with the SNR, although its location within the radio contours may be coincidental. The white cross shows the X-ray coordinates of the SNR centre.

cloud that lies in front of the remnant in the north (as seen clearly in the $160 \mu \mathrm{m}$ emission). The remaining SN-related dust features (1 and 2) originally seen in the $24 \mu \mathrm{m}$ Spitzer data are spatially coincident with the forward shock material regions NW and S in Combi et al. (2016). All of the dust features that are bright in the mid-FIR are anticorrelated with the X-ray ejecta regions indicating the dust in the south and across the centre is simply swept up dust in a shell, with emission appearing to lie interior to the shock wave simply being due to projected emission from the shell (as we also saw in the Tycho SNR; Gomez et al. 2012a).

We note that C19 also found dust associated with another candidate Type Ia SNe G344.7-0.1 (see also Combi et al. 2010a; Giacani et al. 2011; Yamaguchi et al. 2012), where the dust features are also consistent with a non-supernova origin.

G309.8+0.0: There is lots of FIR emission across the region, although the majority seems to be unrelated extended emission from interstellar clouds. We detect a bright knot of $70 \mu \mathrm{m}$ emission to the east which has a similar morphology to the radio structure (indicated by the circle in Fig. 12). However, the FIR extends slightly beyond the radio contour, and the location of this emission may be coincidental. Reach et al. (2006) listed this as a level 3 detection based on NIR Spitzer observations $(3.6-8.0 \mu \mathrm{m})$. The SNR is listed in the table of $\mathrm{OH}$ detections from Green et al. (1997) but whether this originates from a maser has not been confirmed. A bright radio source, thought to be extragalactic in origin (Whiteoak \& Green 1996), is observed to the north of the SN centre at $\alpha=$ $13^{\mathrm{h}} 50^{\mathrm{m}} 35.2^{\mathrm{s}}, \delta=-62^{\circ} 00^{\prime} 42^{\prime \prime}$; no related FIR emission is observed at this location. Given the uncertainty as to whether the dust feature within the gold circle is associated with the ejecta due to the multiple sources seen in the field with similar Herschel colours, we classify this SNR as a level 2 detection.

G350.1-0.3: This source is thought to be a very young corecollapse SNR ( $\leq 1 \mathrm{kyr}$ ) with an associated neutron star (Gaensler et al. 2008; Lovchinsky et al. 2011). It has a distorted clumpy structure with X-ray emission from four regions. Spitzer MIPS observations revealed $24 \mu \mathrm{m}$ emission arising from two of these

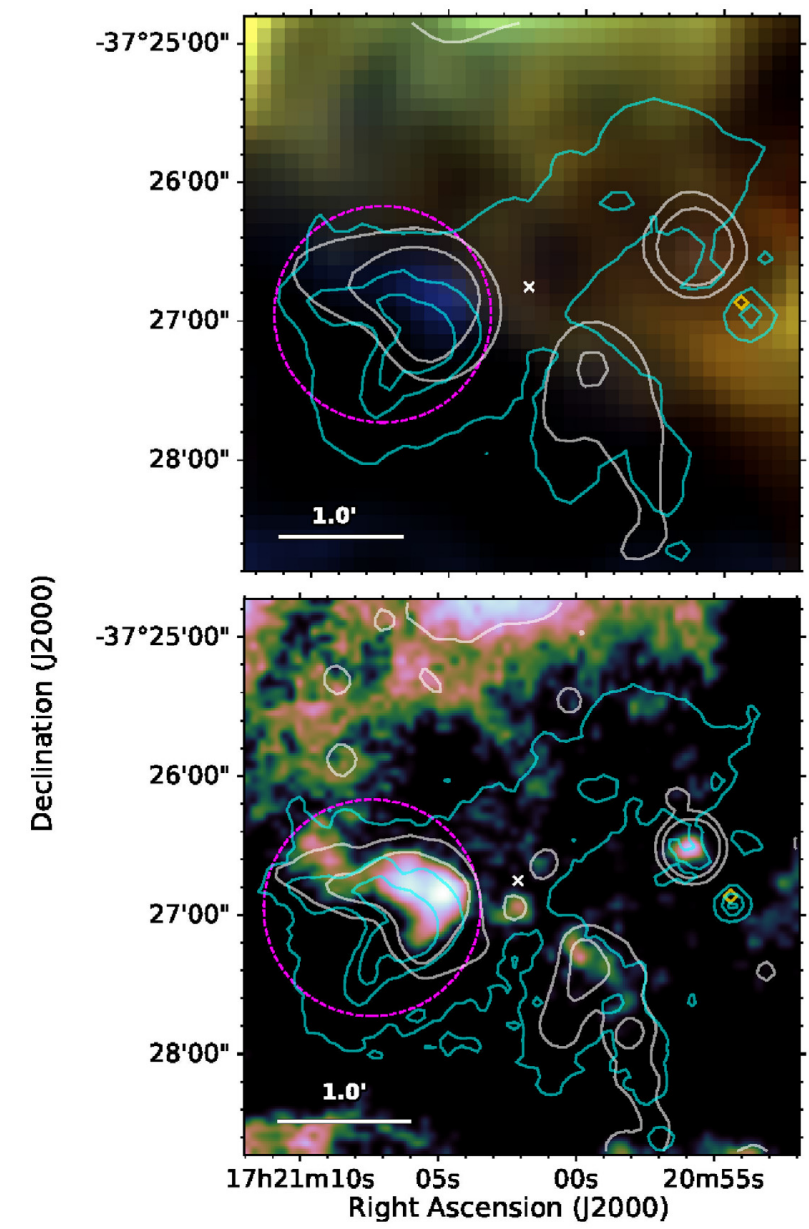

Figure 13. G350.1-0.3 - Top: Herschel three colour image (smoothed to the $250 \mu \mathrm{m}$ resolution). Bottom: Herschel $70 \mu \mathrm{m}$ image. The white contours are $24 \mu \mathrm{m}$ Spitzer MIPS (Lovchinsky et al. 2011) and the cyan contours are $\mathrm{X}$-Ray Chandra. Dust emission is detected to the eastern side of this remnant, within the magenta circle. This region correlates with SNR emission in the X-ray, radio, and MIR (Gaensler et al. 2008; Lovchinsky et al. 2011). The gold diamond indicates the location of the associated neutron star, XMMU J172054.5-372652 (Gaensler et al. 2008). The white cross shows the X-ray coordinates of the SNR centre.

$\mathrm{X}$-ray regions, which was suggested as likely originating from ejecta dust heated by the reverse shock (Lovchinsky et al. 2011). Although Spitzer MIPS did not reveal $70 \mu \mathrm{m}$ emission associated with the SNR structures (Lovchinsky et al. 2011), with the higher resolution and more sensitive Herschel PACS, we have detected dust emission at $70 \mu \mathrm{m}$ in one of the $24 \mu \mathrm{m}$-emitting regions to the east, as indicated by the magenta circle in Fig. 13. We do not see any evidence of associated emission in the longer Herschel wavebands. Since the Herschel $70 \mu \mathrm{m}$ feature overlaps with the $24 \mu \mathrm{m}$ dust attributed to the SNR and with X-rays, we classify this source as a level 1 detection. We do not detect FIR emission at the location of the proposed compact object (CCO) (Gaensler et al. 2008) (indicated by the gold diamond). The strong $70 \mu \mathrm{m}$ emission from the dust region is similar to the properties of the ejecta dust discovered in SNRs G11.2-0.3, G21.5-0.9, G29.7-0.3 (C19), and G54.1+0.3 (Temim et al. 2017; Rho et al. 2018). Therefore G350.1-0.3 can be added to the growing list of SNRs that contain dust within their ejecta. 

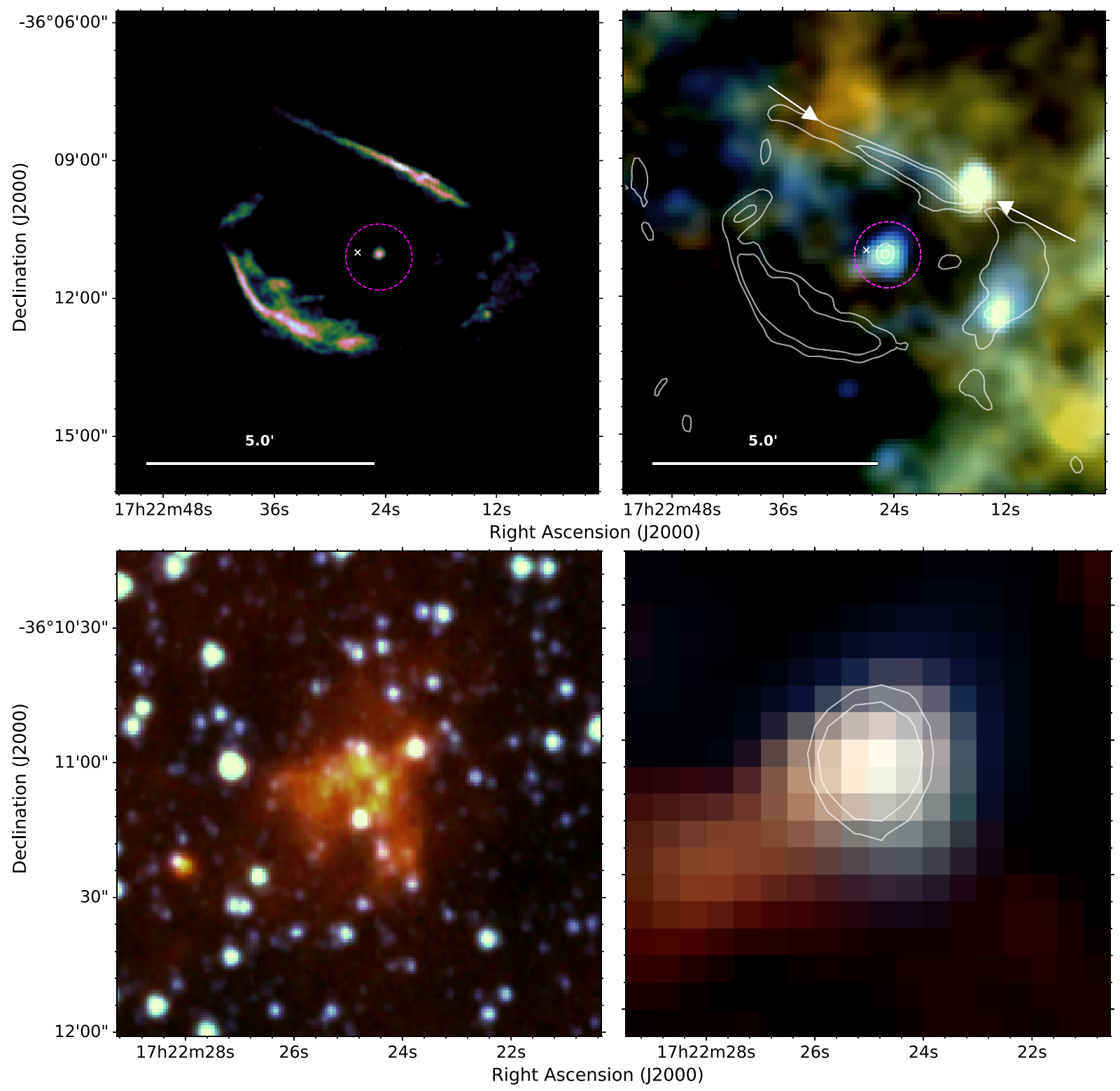

Figure 14. G351.2+0.1 - Top left-hand panel: VLA C-band (4.5-6.4 GHz) image and Top right-hand panel: Herschel three colour image with VLA C-band $(4.5-6.4 \mathrm{GHz})$ contours overlaid. FIR emission is detected in a northern bar, between the two arrows, and more faintly from the south-western shell at 70 um. Emission from the central region within the magenta circle is detected across all Herschel wavebands. The white cross shows the radio coordinates of the SNR centre from Green (2014). Bottom left-hand panel: Three colour IRAC image using bands 1 (3.6 $\mu \mathrm{m}$ - blue), 3 (5.8 $\mu \mathrm{m}$ - green), and 4 ( $8 \mu \mathrm{m}$ - red). This image was made using archive data from the Spitzer Enhanced Imaging Products Super Mosaic Pipeline. Bottom right-hand panel: Herschel three colour image with VLA radio contours (see top panel), note we have changed the colour scaling compared to the top right-hand panel in order to highlight the central features more clearly.

G351.2+0.1: This SNR has a well-defined radio shell (Caswell et al. 1983a), which appears flattened in the north (Dubner et al. 1993) indicating that the shockfront is interacting with dense ISM and that the SNR is relatively young. The SNR has a compact (15 arcsec diameter) central core in the radio (within the magenta circle in Fig. 14) and this is attributed to synchrotron emission powered by a pulsar (Becker \& Helfand 1988). Fig. 14 compares the radio emission at $4.8 \mathrm{GHz}$ (Top lefthand panel) with the Herschel three colour image (Top righthand panel). We processed the radio data presented in Becker \& Helfand (1988) in AIPS, following standard calibration and imaging procedures.
In the Herschel images, we detect emission from the northern shell of the SNR coincident with the thin radio shell seen in the VLA image, as indicated by the arrows in Fig. 14. There is also a bright $70 \mu \mathrm{m}$ clump on the south-western side of the SNR that coincides with a radio peak. We see very little $70 \mu \mathrm{m}$ emission at the location of the south-east shell where the radio is bright. The brightest $70 \mu \mathrm{m}$ emission observed in the SNR is from the central radio core (within the magenta circle, this is also visible in archival Spitzer MIPS $24 \mu \mathrm{m}$ data and the Spitzer IRAC image in Fig. 14). A further bright $70 \mu \mathrm{m}$ source can be seen just north of the flattened northern shell, and a fainter point source that lies to the immediate east of the central core are also detected; these are detected in the 
longer Herschel wavebands and are likely not associated with the SNR. We classify this source as a level 1 detection due to the spatial coincidence of the central FIR core and northern FIR shell with corresponding radio features.

The potential FIR detection of a compact object, or PWN is interesting to explore, although the central core is atypical of its class. It is very faint $(10 \mathrm{mJy}$ at $6 \mathrm{~cm})$ (Becker \& Helfand 1988) with spectral slope $\alpha=+0.27$, where flux varies with frequency as $S_{v} \propto v^{-\alpha}$ suggestive of a very weak source (or possibly old source), compared to Crab-like remnants where typically $\alpha$ ranges from $\sim-0.25$ to -0.3 . The oddness of this source led Becker \& Helfand (1988) to discuss whether it could be a H II region or stellar wind, where the spectral slope would be predicted to be $-0.1(+2.0)$ for optically thin (optically thick) emission or +0.6 , respectively. The source was not detected by the MOST radio survey (Whiteoak \& Green 1996), though it lies below their sensitivity level. Notably, the central core is not detected in Chandra archive observations. ${ }^{1}$ We note that a hard spectrum X-ray source attributed to a pulsar was observed within a $2 \times 4 \mathrm{deg}$ region with GINGA (Tawara et al. 1988) overlapping the location of G351.2+0.1 and with a previously detected X-ray source (GPS1722-363). The pulsation rate of this source is $414 \mathrm{~s}$, oscillating between 1 and 4 milliCrab (where $1 \mathrm{Crab}$ is equivalent to $15 \mathrm{keV} \mathrm{cm}^{-2} \mathrm{~s}^{-1}$ in the $\mathrm{X}$-ray range $2-10 \mathrm{keV})$.

The compact core region is clearly detected in NIR archive images of the region. Fig. 14 (bottom panel) shows a close up view of the NIR and FIR emission in the central core, with NIR Spitzer IRAC three colour image on left $(3.6,5.8$, and $8 \mu \mathrm{m})$, and the FIR Herschel three colour image on the right. The central radio core is an extended feature in the NIR bands, and is very similar to the IRAC images of the PWN G54.1+0.3 and compact PWN in G21.5-0.9 (Zajczyk et al. 2012). In G54.1+0.3, Spitzer IRS spectra revealed that the IRAC feature was from argon line emission from the ejecta and a broad silicate dust feature peaking at $9 \mu \mathrm{m}$ (Temim et al. 2017; Rho et al. 2018). The similarity of the NIR emission features could therefore suggest that G351.2+0.1 adds to the growing list of sources of SN ejecta dust detected from a PWN. We return to this source in Section 3.4.

G354.1+0.1: There are multiple radio features in this region and there are FIR filaments of emission observed in the same regions as radio structures. Assuming that the radio emission from the SNR is the region encompassing the pulsar (see Fig. 15), the FIR may be associated with the SNR, although we cannot definitively determine an association due to the numerous unrelated FIR sources in this region (gold circles in Fig. 15). Thus we classify this as a level 2 detection. We do not detect emission at the location of the associated pulsar, PSR 1727-33, indicated by the gold diamond in Fig. 15 (Frail, Goss \& Whiteoak 1994).

G355.6-0.0: This SNR is another mixed morphology source (Rho \& Petre 1998). As seen in Fig. 16, radio observations show a clear shell in the MOST $843 \mathrm{MHz}$ image (Gray 1994b; their fig. 2) with enhanced emission to the west. There are multiple FIR filaments in the region of this SNR, although the majority do not seem to correlate with the radio. There is a filament of emission near $\alpha=17^{\mathrm{h}} 35^{\mathrm{m}} 08^{\mathrm{s}}, \delta=-32^{\circ} 36^{\prime} 14^{\prime \prime}$ which may be associated with the radio peak at the western edge of the SNR, although the region is confused and we cannot confidently determine whether this is related. This satisfies our criteria for a level 2

\footnotetext{
${ }^{1}$ Chandra maps (obsid 3844; 4591) covering the SNR footprint are presented
} in the massive star forming region omnibus of Townsley et al. (2018)

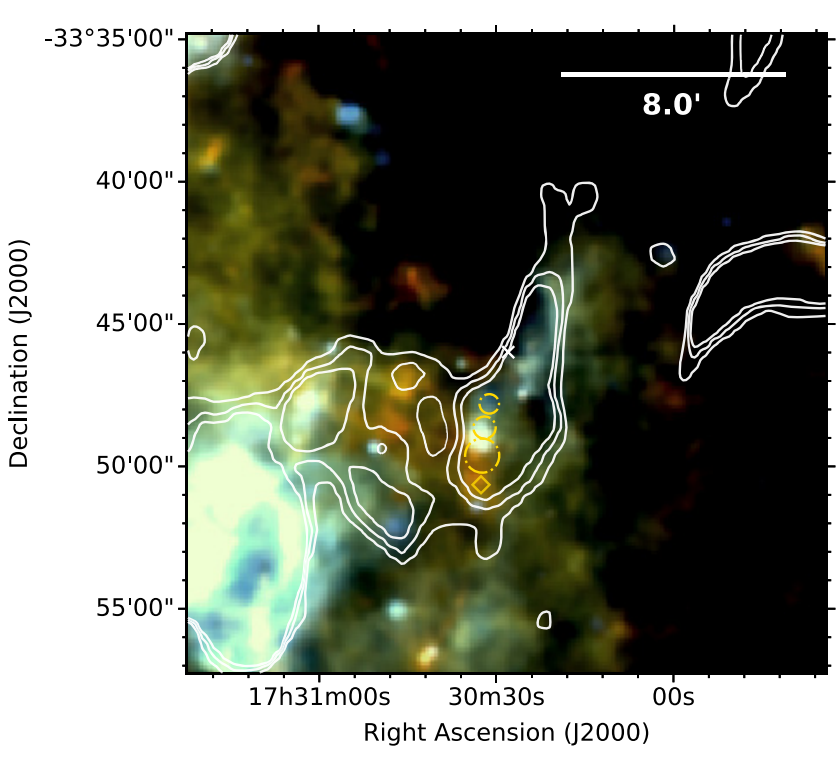

Figure 15. G354.1+0.1 - Herschel three colour image with MOST $843 \mathrm{MHz}$ radio contours overlaid. FIR emission is detected from within the radio contours although association with the SNR is unclear. The gold circles indicate the locations of bubbles (Simpson et al. 2012) and a dense core (Purcell et al. 2012). The white cross shows the radio coordinates of the SNR centre from Green (2014).

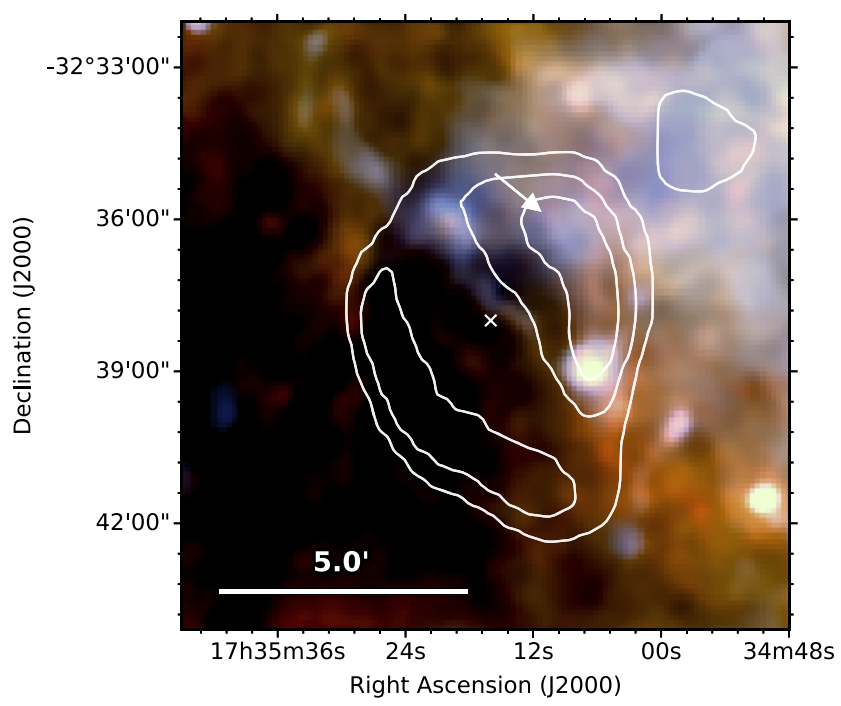

Figure 16. G355.6-0.0 - Herschel three colour image with MOST $843 \mathrm{MHz}$ radio contours overlaid. There is FIR emission, with strong $70 \mu \mathrm{m}$ flux indicated by the arrow, which may be related with SNR radio structure, although the region is extremely confused and any SNR emission is difficult to disentangle. The white cross shows the radio coordinates of the SNR centre from Green (2014).

detection in that there is potential FIR emission related to $\mathrm{SN}$ features.

G357.7-0.1 (MSH 17-39): Known as 'the Tornado', this unusual SNR candidate comprises a 'head', which appears as a shell- or ring-like feature in the radio (Shaver et al. 1985) and an unresolved extended clump in X-rays (Gaensler et al. 2003), a larger extended radio shell/filamentary structure, and an elongated 'tail'. These features are marked with a magenta circle, contours, and arrows, respectively, in Fig. 17. The compact, bright radio source seen to 


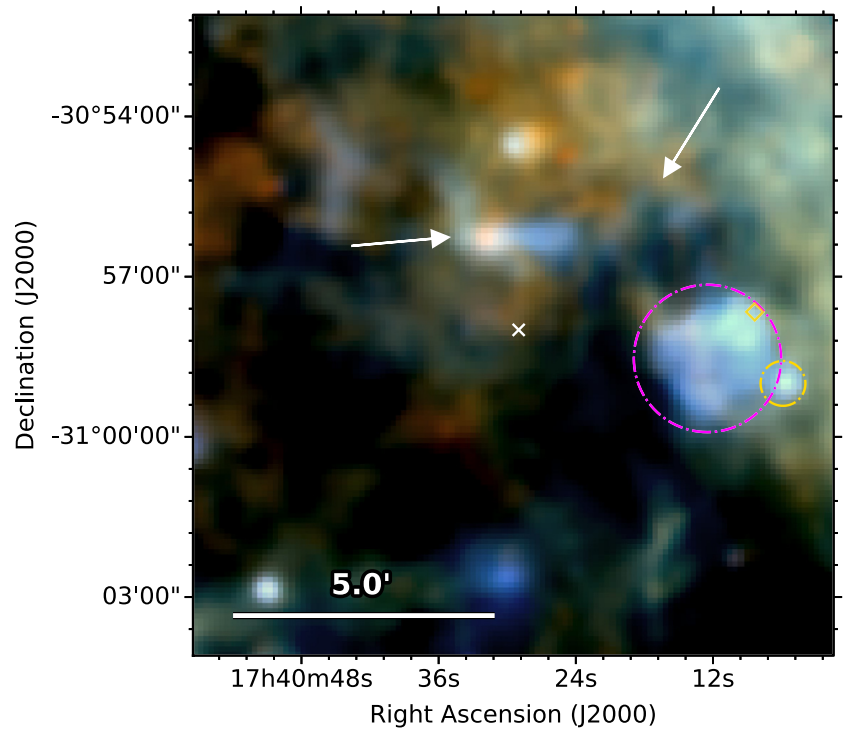

Figure 17. G357.7-0.1, The Tornado - Herschel three colour image. We detect dust emission across all Herschel wavebands for the 'head' of The Tornado, within the magenta circle. We also detect FIR emission from the 'tail' of The Tornado, and from a fainter filament extending around the head, as indicated by the arrows. The H II region known as the 'eye' of The Tornado is detected to the west of the 'head', within the gold circle. The gold diamond indicates the location of an $\mathrm{OH}(1720 \mathrm{MHz})$ maser. The white cross shows the radio coordinates of the SNR centre from Green (2014).

the west of the head at $\alpha=17^{\mathrm{h}} 40^{\mathrm{m}} 05.9^{\mathrm{s}}, \delta=-30^{\circ} 59^{\prime} 00^{\prime \prime}$ is the so-called 'eye' of the Tornado. However, this has been shown to be unrelated to the SNR structure and is proposed to be an isolated core embedded in a foreground H II region (Brogan \& Goss 2003; Burton et al. 2004).

Although this region is confused in the FIR (Fig. 17), the Herschel images show clear emission from dust, with bluer colours than the surrounding interstellar material, in the Herschel images at the location of the head and the tail. The coincidence of FIR emission with the radio-bright head makes this a level 1 detected source. Further analysis of this source can be found in Chawner et al. (in preparation).

G357.7+0.3: This source has a distinctive square morphology giving rising to the name 'the Square Nebula'. There is extended $\mathrm{OH}$ (1720 MHz) emission along the western edge of the SNR (YusefZadeh \& Goss 1999) and the recent discovery of shocked molecules, broad Co in millimetre, shocked $\mathrm{H}_{2}$ lines with Spitzer, and $158 \mu \mathrm{m}$ [C II] with SOFIA in Rho et al. (2017) provides unambiguous evidence that this SNR is interacting with a molecular cloud. Although there is lots of extended FIR structure making associated emission difficult to distinguish, there are filaments at the western edge near to the $\mathrm{OH}(1720 \mathrm{MHz})$ emission which may be related. We therefore classify this as a level 2 detection.

Phillips et al. (2009) observed comet-like structures to the west of this source which they suggest result from interactions between the SNR and nearby molecular clouds. We detect FIR emission across all Herschel wavebands from these structures near to $\alpha=$ $17^{\mathrm{h}} 36^{\mathrm{m}} 57^{\mathrm{s}}, \delta=-30^{\circ} 46^{\prime} 02^{\prime \prime}, \alpha=17^{\mathrm{h}} 35^{\mathrm{m}} 59^{\mathrm{s}}, \delta=-30^{\circ} 53^{\prime} 03^{\prime \prime}$, and $\alpha=17^{\mathrm{h}} 36^{\mathrm{m}} 11^{\mathrm{s}}, \delta=-31^{\circ} 07^{\prime} 40^{\prime \prime}$, indicated by the gold circles in Fig. 18. We also see a plume of dust emission near the Mira star V1139 Sco, originally seen at $24 \mu \mathrm{m}$ in Phillips et al. (2009). The origin of this emission is unclear; Phillips et al. (2009) propose that the wind is being swept behind the star by an interaction

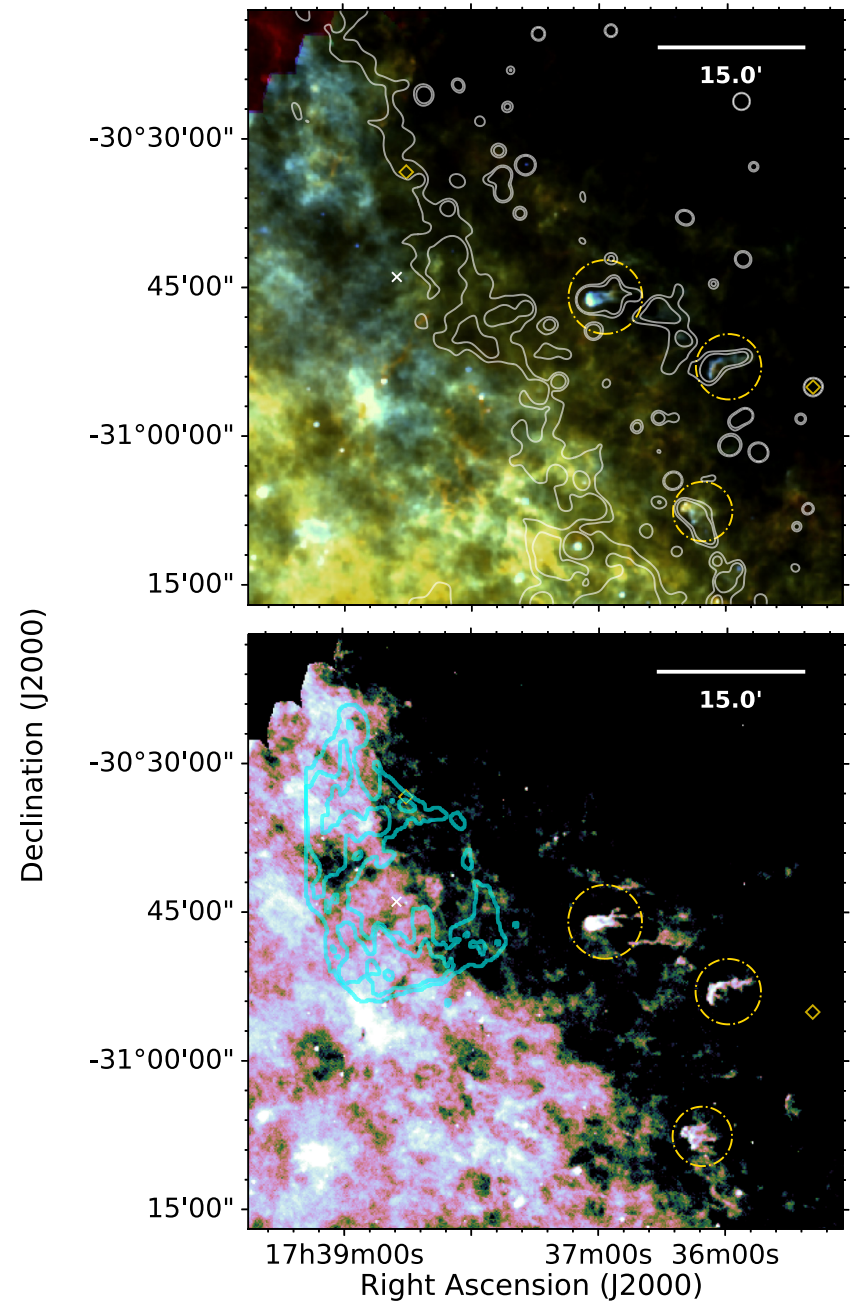

Figure 18. G357.7+0.3 - Top: Herschel three colour image with MIPS $24 \mu \mathrm{m}$ contours overlaid. Bottom: Herschel $70 \mu \mathrm{m}$ image with MOST $843 \mathrm{MHz}$ contours overlaid (radio image is shown in fig. 2 from Gray 1994a and fig. 1 from Rho et al. 2017). FIR emission is observed within the gold circles from 'comet' like structures to the west detected by Phillips et al. (2009), we do not expect that these structures are associated with the SNR. The white cross shows the radio coordinates of the SNR centre from Green (2014). The gold diamond to the west indicates the location of the Mira star V1139 Sco and the diamond in the north indicates the location of an $\mathrm{OH}$ $(1720 \mathrm{MHz})$ maser.

with the SNR, although the Mira star may be unrelated and its location coincidental. We do not expect that any of these structures are associated with the SNR. If the structures were the result of interactions with the expanding SNR we would expect them to point away from it and the brightest emission would be at the furthest point from the remnant centre. The morphology of these objects is more similar to evaporating gaseous globules (EGGs) or protoplanetary discs, it is therefore more likely that the knots are associated with star formation, which may have been triggered by the SN. In order to check whether the comet-like structures could be a result of the SNR interaction, we roughly estimate the distance to these regions and compare with the distance travelled by the SNR shock. We note that little is known about the progenitor for this source. We focus on the structures labelled A to D in fig. 2 of Phillips et al. (2009) and the closest source to the SNR, structure G357.46+0.60. To be consistent with Phillips et al. (2009) we assume that the SNR 


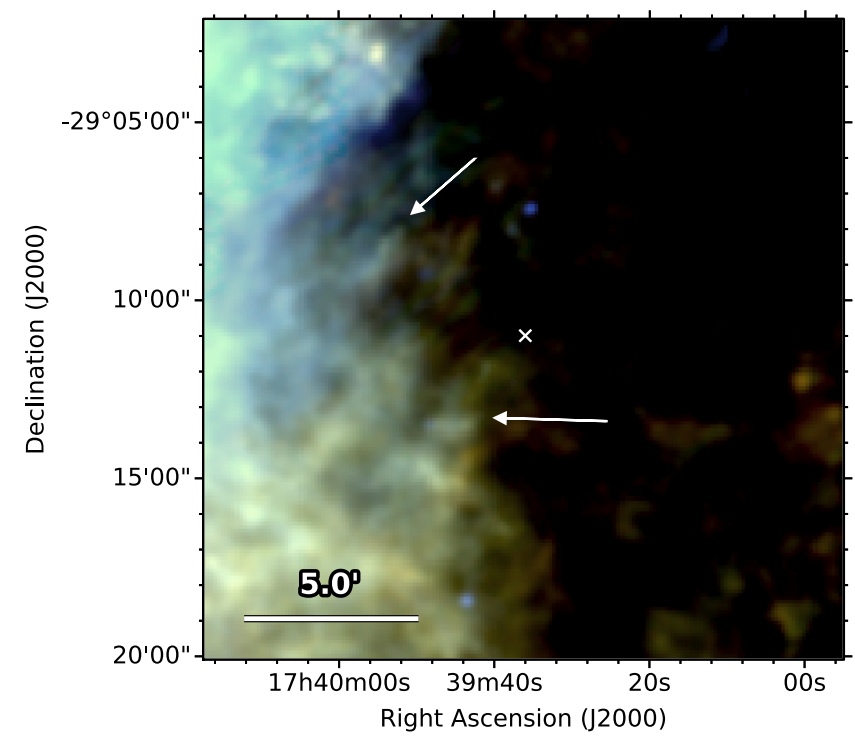

Figure 19. G359.1+0.9 - Herschel three colour image. There are FIR filaments towards the western edge of the SNR which may be associated although the region is very confused. The white cross shows the radio coordinates of the SNR centre from Green (2014).

is at $4 \mathrm{kpc}$, and then assume that the comet-like structures are at the same distance. Thus the closest edge of the structures are at distances between 22 and $51 \mathrm{pc}$ from the radio centre of the SNR (from Green 2014). The distance reached by the shock front is estimated initially by assuming the most optimistic case, whereby the SNR is freely expanding since the explosion (i.e. it is not yet in the Sedov-Taylor phase). Assuming an ejecta mass between 5 and $15 \mathrm{M}_{\odot}$ and an age of $<6400 \mathrm{yr}$ (Phillips et al. 2009), the shock wave would have reached a maximum distance of $\sim 29.3 \mathrm{pc}$ in this time. We find that only the closest globule (G357.46+0.60) is within range of the shockwave, and only if the ejected mass is smaller than $8.5 \mathrm{M}_{\odot}$. If we assume the SNR is in the Sedov-Taylor expansion phase, the SF globules are well outside of the estimated shock wave radius $(5.0 \mathrm{pc})$ for a typical ISM $\mathrm{n}(\mathrm{H})$ of $1 \mathrm{~cm}^{-3}$. Higher interstellar densities would further reduce the reach of the SNR. Thus we do not expect that any of these structures are associated with the SNR.

Phillips et al. (2009) used MIPS $24 \mu \mathrm{m}$ combined with low (arcmin) resolution IRAS data to propose that the northern-most of these structures contains dust at $\sim 30 \mathrm{~K}$. Fitting a modified blackbody to background subtracted Herschel fluxes (see C19 for details) confirms that it contains dust at $\sim 33 \mathrm{~K}$.

G359.1+0.9: This SNR has a shell-like structure in the radio (Gray 1994b; their fig. 10). Comparing the Herschel emission by eye with their figure, ${ }^{2}$ we see bright extended emission to the east of this source and multiple FIR filaments at the location of the SNR. Some of these filaments may be associated with the SNR, although Fig. 19 shows that this region is very confused and we cannot definitively distinguish SNR and unrelated emission making this a level 2 detection.

In the following section, we look at some of the properties of the dust emission detected in the level 1 SNRs.

${ }^{2}$ unfortunately, the radio fits data for this source or region was not available for download online.

\section{INVESTIGATING DUST PROPERTIES IN GALACTIC SUPERNOVA REMNANTS}

Here we investigate the properties of the dust within a sample of our level 1 detected SNRs from both this work and C19. We search for detection level $1 \mathrm{SNRs}$ that are not in overly complex/crowded regions of the Galactic Plane, and sources that have not previously been studied in detail (this excludes G11.2-0.3, G21.5-0.9, G29.7-0.3 (Chawner et al. 2019), G54.1+0.3 (Temim et al. 2017; Rho et al. 2018), and G357.7-0.1 (Chawner et al. in preparation). This leaves us with a total of 11 sources out of the 39 level 1 detected SNRs.

First we investigate the dust temperatures in the SNRs. Dust heated by the PWN, a reverse shock, or a forward shock in a supernova remnant should lead to differences in dust temperatures compared to that in the surrounding interstellar medium since shocks lead to high temperature electrons which can collisionally heat dust grains (Dwek \& Arendt 1992). We create temperature maps of the dust in these SNRs (simulations of the temperature maps are discussed in full in Appendix B1) after estimating the background flux using the NEBULIZER routine, ${ }^{3}$ which subtracts medium to large-scale variations in the background of astronomical images.

\subsection{Background subtraction}

A large number of our level 1 detected sample are in complicated regions of the ISM and have large variations in the source flux. We minimize this issue by subtracting contamination from the ISM using the NEBULIZER routine. NEBULIZER estimates medium to large-scale variations in the background which can be subtracted from our SNR regions to give a more accurate background-subtracted map. ${ }^{4}$ As demonstrated in Appendix B1, this can be used to accurately determine the properties of source-related structures.

Many of our level 1 detected SNRs are in crowded fields and are close to or interacting with unrelated structures such as H II regions. We cannot subtract these using NEBULIZER without also losing information about the SNR and our resultant temperature maps are sensitive to some contamination from such sources. This difficulty is aggravated as the ISM is bright at Herschel wavelengths, especially $160-500 \mu \mathrm{m}$, making some SNRs difficult to distinguish in any other than the $70 \mu \mathrm{m}$ waveband. Consequently, we find that the NEBULIZER background subtraction at $160 \mu \mathrm{m}$ is too harsh in some regions, although this effect is much reduced compared with for example, if we were to simply subtract a constant value across the entire region.

\subsection{Flux estimates}

After subtracting the medium large-scale background with NEBULIZER we estimate the FIR flux from each SNR in five Herschel

\footnotetext{
${ }^{3} \mathrm{http} / / /$ casu.ast.cam.ac.uk/surveys-projects/software-release/backgroundfiltering

${ }^{4}$ In brief, the Nebulizer algorithm takes a square of $N$ by $N$ pixels around a pixel and estimates the median of the intensities centred on that pixel to estimate the background at that position. The background map is smoothed using a box-car mean filter with box size chosen to be $N / 2 \times N / 2$ (Irwin 2010). The value of $N$ used varies between 75 and 350 , depending on fluctuations in the background and size of the SNR, and in some cases we mask bright sources to avoid overestimating the background
} 
wavebands. For several of our sample there is some contamination remaining in the background-subtracted maps as there is structure of a similar scale to the SNR which is difficult to remove. In these cases we apply a secondary subtraction estimated from the average level using an annulus. The fluxes for our 11 SNRs are shown in Table 2 .

The uncertainty associated with the flux is estimated using a combination of the Herschel calibration uncertainty, variation of the NEBULIZER background map within the aperture, and, where applicable, variation within the annulus used for a secondary background subtraction. In these sources the contaminating ISM flux may act to decrease our estimates of the dust temperature, nevertheless, this effect will be much reduced compared with temperatures estimated without any background subtraction, as seen in Fig. 20.

\subsection{Temperature and mass maps of Milky Way SNRs}

The three colour images created out of the Herschel images shown in Section 2.1 and Fig. A1 reveal dusty structures and features that appear to be associated with other known SNR tracers e.g. radio, $\mathrm{X}$-ray, or optical emission (i.e. det $=1$ ) or potentially related to the $\mathrm{SN}$ (i.e. det $=2$ ). The colour of the dust emission in these images is, in effect, tracing the dust temperature in the environs of the SNR; however the colour scales chosen earlier are arbitrary, selected only to highlight dusty features. Here we attempt to investigate the temperature of SNR-related dust and potential heating sources using the FIR flux ratio $(70 \mu \mathrm{m} / 160 \mu \mathrm{m})$ seen by Herschel for the detected level 1 sources (following similar studies that used $24 \mu \mathrm{m} / 70 \mu \mathrm{m}$ flux ratios, e.g. Sankrit et al. 2010; Pinheiro Goncalves et al. 2011; Williams et al. 2011; Lakićević et al. 2015; Temim et al. 2015; Koo et al. 2016). We select this colour ratio as this should be sensitive to dust emission from grains at temperatures of $\sim 15-70 \mathrm{~K}$. It should also be less affected by contamination from ionic line emission, which can account for a non-negligible proportion of the $24 \mu \mathrm{m}$ flux (e.g. Koo et al. 2016; De Looze et al. 2019) and less affected by unrelated point sources seen in $24 \mu \mathrm{m}$ images. However, this ratio, and particularly the $160 \mu \mathrm{m}$ band, may be affected by confusion with unrelated interstellar dust and line emission ([C II $158 \mu \mathrm{m}$ ). The temperature maps are derived using the 70 and $160 \mu \mathrm{m}$ NEBULIZER subtracted maps and assuming a single temperature dust component with power-law dust opacity index $\beta=1.9$ (see C19 for more

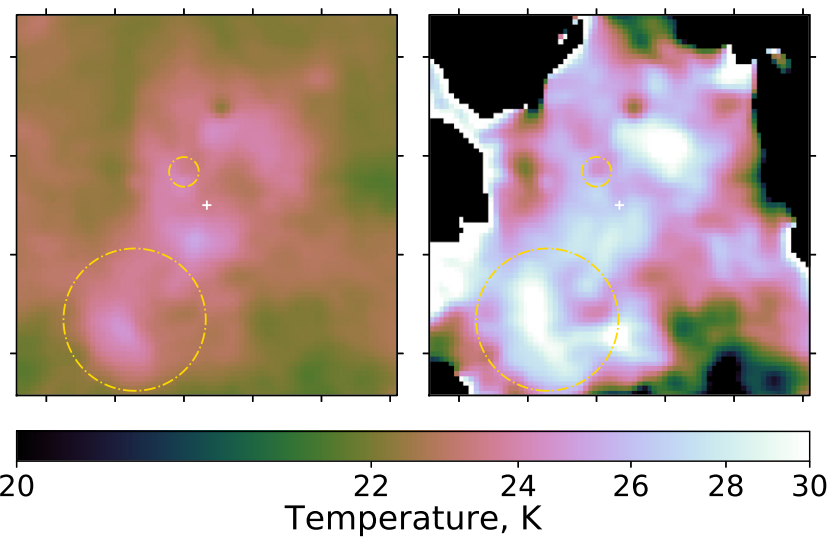

Figure 20. Left-hand panel: Dust temperature map of the level 1 detected SNR G8.3-0.0 (Fig. 3), derived using equation (1) without background subtraction. Right-hand panel: Dust temperature map derived after using Nebulizer to subtract the background. The Herschel images used to create the right-hand figure have been convolved to the $250 \mu \mathrm{m}$ resolution to improve the signal. We are clearly able to identify a wider range of temperatures after applying Nebulizer background subtraction. The large and small gold circles indicate the locations of an IR bubble and a maser, respectively.

details) using the equation:

$$
\frac{F_{70}}{F_{160}}=\left(\frac{\lambda_{160}}{\lambda_{70}}\right)^{\beta+3} \frac{\exp \left(\frac{h c}{\lambda_{160} k T}\right)-1}{\exp \left(\frac{h c}{\lambda_{70} k T}\right)-1},
$$

where $F_{70}$ and $F_{160}$ are the fluxes at 70 and $160 \mu \mathrm{m}, \lambda$ is the wavelength, $h$ is the Planck constant, $c$ if the speed of light, $k$ is the Boltzmann constant, and $T$ is the dust temperature.

We present the dust temperature maps in Fig. 21 with individual notes discussing the map for each SNR provided in Appendix B2. An example of the temperature maps derived for the SNR G8.3-0.0 before and after background subtraction is shown in Fig. 20 to illustrate the significant underestimation of the dust temperature (thus leading to an overestimation of the dust mass) if the background level is not properly accounted for. Before subtraction only a narrow range of dust temperatures are seen in the SNR $\left(T_{d}<26 \mathrm{~K}\right)$, whereas after subtraction dust is seen at $26<T_{d}<30 \mathrm{~K}$. See also the recent reductions of the proposed SN dust mass in the Crab Nebula due to more sophisticated measures of the background level

Table 2. The SNR flux is measured within the dashed white circle in Fig. 21 from background subtracted maps convolved to the resolution of the $500 \mu \mathrm{m}$ Herschel map. In some cases point sources within the aperture are masked before measuring the flux. Uncertainties are estimated from the standard deviation of the background map within the aperture and the Herschel calibration uncertainty.

\begin{tabular}{|c|c|c|c|c|c|}
\hline \multirow[t]{2}{*}{ SNR } & \multicolumn{5}{|c|}{ Flux (Jy) } \\
\hline & $70 \mu \mathrm{m}$ & $160 \mu \mathrm{m}$ & $250 \mu \mathrm{m}$ & $350 \mu \mathrm{m}$ & $500 \mu \mathrm{m}$ \\
\hline G8.3-0.0 & $1054.8 \pm 75.8$ & $1898.4 \pm 134.6$ & $883.7 \pm 49.1$ & $370.2 \pm 20.5$ & - \\
\hline $\mathrm{G} 11.1+0.1$ & $1251.0 \pm 674.6$ & $2934.8 \pm 2549.5$ & $2142.4 \pm 1794.6$ & $1010.3 \pm 766.3$ & $425.2 \pm 298.1$ \\
\hline G16.4-0.5* & $2766.2 \pm 1211.3$ & $8907 \pm 4054.2$ & $6581.5 \pm 1767.6$ & $2964.0 \pm 1139.9$ & $1204.7 \pm 320.6$ \\
\hline G20.4+0.1* & $979.9 \pm 241.4$ & $1508.2 \pm 918.1$ & $897.0 \pm 607.0$ & $377.7 \pm 249.9$ & $148.2 \pm 95.2$ \\
\hline $\mathrm{G} 21.5-0.1^{*}$ & $490.9 \pm 62.4$ & $1050.6 \pm 241.4$ & $697.4 \pm 164.3$ & $302.9 \pm 68.9$ & $119.5 \pm 26.7$ \\
\hline G43.3-0.2 & $744.5 \pm 61.2$ & $283.6 \pm 135.2$ & $74.4 \pm 113.7$ & $20.9 \pm 53.8$ & $5.3 \pm 23.1$ \\
\hline G65.8-0.5* & $75.8 \pm 6.4$ & $96.8 \pm 20.0$ & $22.3 \pm 18.8$ & $8.3 \pm 9.7$ & $2.0 \pm 4.0$ \\
\hline G67.8+0.5 & $107.3 \pm 7.7$ & $203.6 \pm 20.5$ & $100.9 \pm 10.4$ & $46.7 \pm 4.9$ & $18.2 \pm 1.9$ \\
\hline G310.8-0.4 & $1729.9 \pm 593.5$ & $2145.1 \pm 1507.5$ & $935.4 \pm 1018.7$ & $363.9 \pm 449.2$ & $162.4 \pm 183.9$ \\
\hline G340.6+0.3 & $220.6 \pm 29.4$ & $755.0 \pm 117.8$ & $506.5 \pm 85.1$ & $207.5 \pm 36.7$ & $80.2 \pm 14.1$ \\
\hline $\mathrm{G} 349.7+0.2$ & $588.4 \pm 41.5$ & $456.5 \pm 32.8$ & $158.8 \pm 9.1$ & $60.1 \pm 3.5$ & $19.6 \pm 1.2$ \\
\hline
\end{tabular}

Note. ${ }^{*}$ Sources which may be confused with a H II region (Anderson et al. 2017; Gao et al. 2019). 

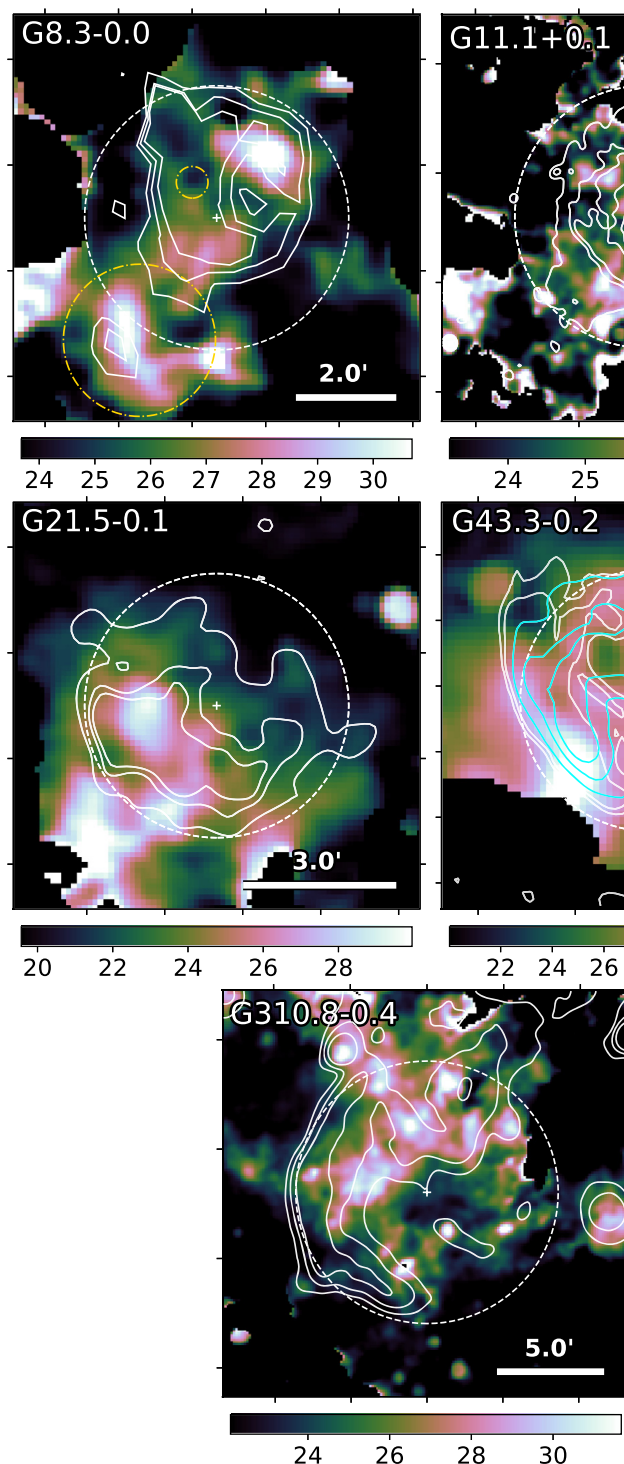
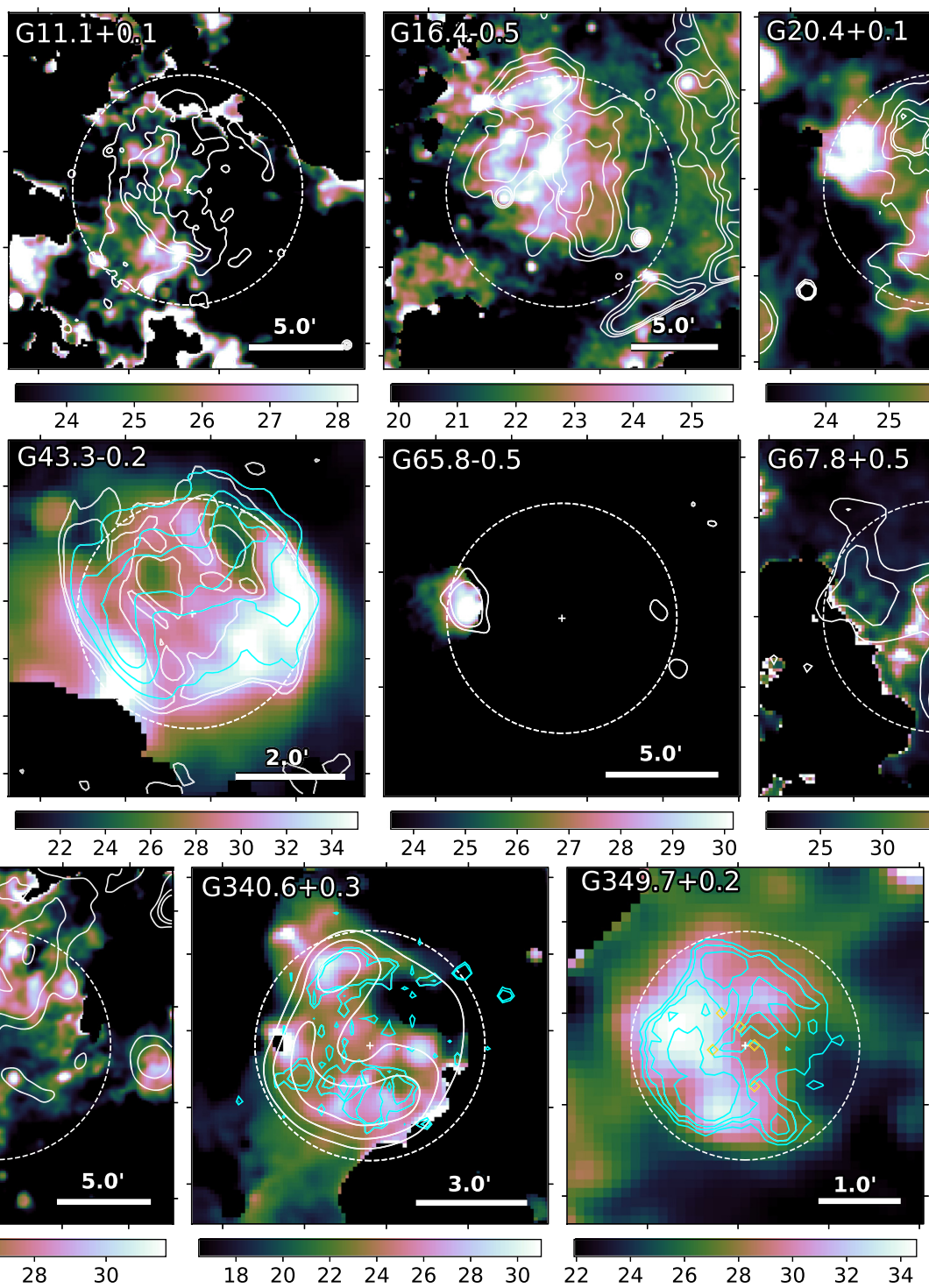

$\begin{array}{lllllll}18 & 20 & 22 & 24 & 26 & 28 & 30\end{array}$
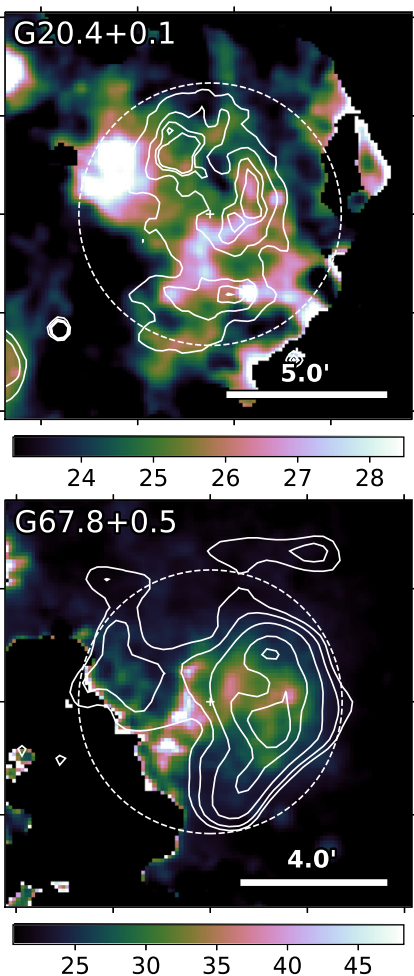

Figure 21. Dust temperature maps of our subset of detected level 1 sources derived using equation (1). The images have been convolved to 6 arcsec to improve the signal. VLA GPS $20 \mathrm{~cm}$ contours (white) are overlaid on to images of G8.3-0.0, G11.1+0.1, G16.4-0.5, G20.4+0.1, G21.5-0.1, and G43.3-0.2. NVSS $1.4 \mathrm{GHz}$ contours (white) are overlaid on to images of G65.8-0.5 and G67.8+0.5. MOST 843 GHz contours (white) are overlaid on to images of G310.8-0.4 and G340.6+0.3. Chandra contours (cyan) are overlaid on to the images of G43.3-0.2, G340.6+0.3, and G349.7+0.2. In some cases oversubtraction of the $160 \mu \mathrm{m}$ background introduces hot artefacts at the edges of masked areas.

(De Looze et al. 2019; Nehmé, Kassounian \& Sauvage 2019). We attempt to interpret the dust temperatures in Section 4.2.

We note that the NEBULIZER routine used to subtract the background finds medium to large ISM variations, but may not subtract smaller scale complicated structure. Therefore, there may be some contamination remaining, which will act to artificially reduce the temperature. For two sources (G43.3-0.2 and G349.7-0.2) we find lower dust temperatures than Koo et al. (2016) derived using the 24-70 $\mu \mathrm{m}$ flux ratio, this may be due to differences in the methods of analysis and/or line contamination in the $24 \mu \mathrm{m}$ waveband. Conversely, in some regions the background may be oversubtracted where there are small-scale variations, this seems to be more of an issue for the $160 \mu \mathrm{m}$ images and this leads to hot artefacts in the maps.

Next we determine dust masses for the SNRs from this sample for which there are estimated distances in the literature. Table 2 shows that, even for our clearest detections, the SPIRE fluxes for our SNRs are very uncertain and we cannot confidently use these fluxes to estimate a dust mass via modified blackbody fits to the IR-submillimetre SED for these sources. Nevertheless, all of our level 1 sources are detected at $70 \mu \mathrm{m}$, we therefore use these maps combined with the temperature information in Fig. 21 to estimate the dust mass within each source, giving the dust maps in Fig. 22. When estimating the mass from these sources there are two issues to resolve. First, there are large areas with relatively small dust mass which, when summed, contribute a large mass to our final estimate. Secondly, in the case of G43.3-0.2 there is a region to the north of the SNR which has a low temperature in Fig. 21 because of low $70 \mu \mathrm{m}$ emission in this region (relative to the $160 \mu \mathrm{m}$ flux), resulting in a large dust mass. To overcome both issues we set a $1.5 \sigma$ threshold on the $70 \mu \mathrm{m}$ background subtracted flux, below which pixels are excluded from our dust mass estimate (lower flux thresholds are required for G43.3-0.2 and G340.6+0.3). Using the white apertures shown in Fig. 22 we find the dust masses given in Table 3. 

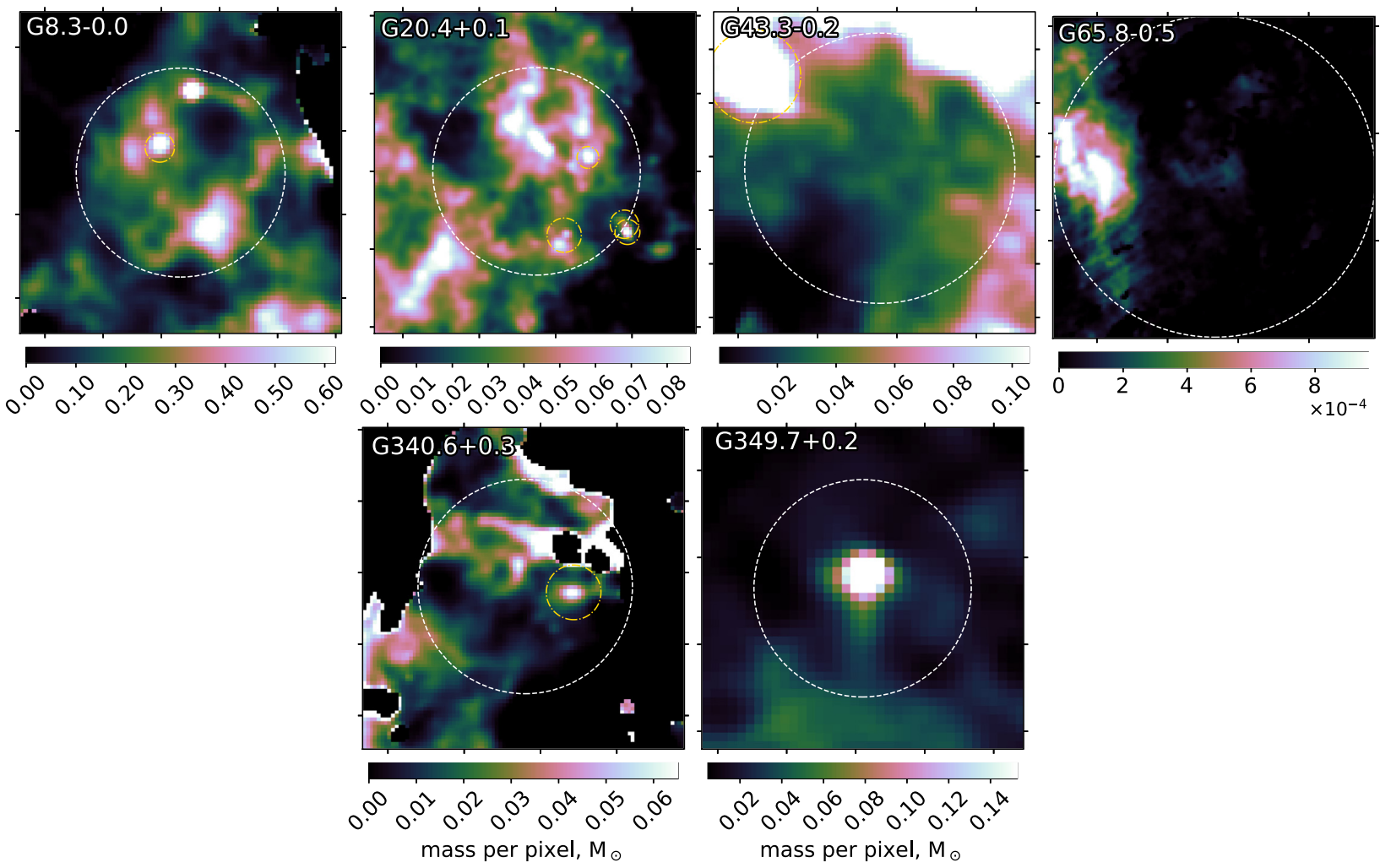

Figure 22. Dust mass maps for our subset of detected level 1 sources, derived using the background subtracted $70 \mu \mathrm{m}$ flux and the temperature maps in Fig. 21. The estimated dust mass for each SNR, as given in Table 2, is estimated from within the white circle for all pixels which have a $70 \mu \mathrm{m}$ flux above a threshold level, after masking unrelated sources (shown by the gold circles).

Table 3. Sources* which may be confused with a H II region (Anderson et al. 2017; Gao et al. 2019). Distance references: 1 - Kilpatrick, Bieging \& Rieke (2016), 2-Ranasinghe \& Leahy (2018b), 3-Ranasinghe \& Leahy (2018a), 4-Shan et al. (2018), 5 - Kothes \& Dougherty (2007), 6-Tian \& Leahy (2014).

\begin{tabular}{lccc}
\hline SNR & Distance $(\mathrm{kpc})$ & Dust mass $\left(\mathrm{M}_{\odot}\right)$ & Refs \\
\hline G8.3-0.0 & 16.4 & 340 & 1 \\
G11.1+0.1 & - & - & - \\
G16.4-0.5* & - & - & - \\
G20.4+0.1* & 7.8 & 136 & 2 \\
G21.5-0.1* & - & - & - \\
G43.3-0.2 & 11.3 & 28.4 & 3 \\
G65.8-0.5* & 2.4 & 0.7 & 4 \\
G67.8+0.5 & - & - & - \\
G310.8-0.4 & - & - & - \\
G340.6+0.3 & 15 & 138 & 5 \\
G349.7+0.2 & 11.5 & 22.5 & 6 \\
\hline
\end{tabular}

We estimate unexpectedly large dust masses for three of our SNRs (G8.3-0.0, G20.4+0.1, and G340.6+0.3). In two of these cases the estimated distance is extremely large; if this is overestimated it will increase our dust mass, i.e. increasing the estimated distance by a factor of 2 would increase the estimated dust mass by a factor of 4. However, we also note that closer remnants will have interacted with a smaller volume of the ISM, and consequently would have conversely swept up less dust mass as their shock front moves out. It is therefore likely that there is another factor contributing to this issue. As discussed previously, our images are highly confused and, despite careful background subtraction, there may still be contaminating ISM which acts to reduce the estimated temperatures in Fig. 21. In the case of G8.3-0.0, increasing the temperature of each pixel by $2 \mathrm{~K}$ or $10 \mathrm{~K}$ reduces our dust mass from $340 \mathrm{M}_{\odot}$ to $\sim 190$ or $35 \mathrm{M}_{\odot}$. Evidently, our dust mass estimates are highly sensitive to small changes in the temperature and we expect that our results are greatly dependent on contaminating ISM.

Previous IRAS surveys by Arendt (1989) and Saken et al. (1992) estimated dust masses for W49B (G43.3-0.2) of 127.7 and $500 \mathrm{M}_{\odot}$ and for $\mathrm{G} 349.7+0.2$ of $<25.1$ and $4900 \mathrm{M}_{\odot}$, which are considerably larger than those that we estimate (although we are consistent with the G349.7+0.2 mass estimated by Arendt 1989). It is clear that background subtraction is crucial in finding accurate dust masses and even small variations in the method of subtraction can give vastly different results. With the higher angular resolution of Herschel compared with IRAS we make drastic improvements in reducing the ISM contamination.

\subsection{The nature of the centre of G351.2+0.1}

In the previous section, we did not produce a dust temperature and mass map for the SNR G351.2+0.1 due to the large gradient in interstellar dust seen in the immediate vicinity of the SNR shell (Fig. 14). However the inner central/radio bright region where we discovered dust emission is observed at high $\mathrm{S} / \mathrm{N}$ in all of the Herschel bands. This potentially indicates the discovery of FIR emission coincident with a possible Crab-like compact object. To determine the nature of this emission across the NIR - radio regime and because of its brightness in all bands, for this source, we create 
Table 4. Background subtracted flux measurements for the compact source at the centre of G351.2+0.1. IRAC fluxes have been dereddened (Indebetouw et al. 2005) and point sources in the Spitzer IRAC and MIPS $24 \mu \mathrm{m}$ images were masked out.

\begin{tabular}{lccc}
\hline & NIR - FIR & \multicolumn{2}{c}{ Radio } \\
Wavelength & $\begin{array}{c}\text { Flux } \\
\text { Jy }\end{array}$ & $\begin{array}{c}\text { Frequency } \\
\text { GHz }\end{array}$ & $\begin{array}{c}\text { Flux } \\
\text { Jy }\end{array}$ \\
\hline 3.6 & $0.143 \pm 0.022$ & 15 & $0.0124 \pm 0.0002$ \\
4.5 & $0.095 \pm 0.014$ & 5 & $0.0090 \pm 0.0003$ \\
5.8 & $0.579 \pm 0.087$ & 1.5 & $0.0064 \pm 0.0010$ \\
8.0 & $1.590 \pm 0.239$ & - & - \\
24 & $1.155 \pm 0.041$ & - & - \\
70 & $26.960 \pm 2.716$ & - & - \\
160 & $20.256 \pm 2.496$ & - & - \\
250 & $4.469 \pm 2.631$ & - & - \\
350 & $0.292 \pm 1.966$ & - & - \\
\hline
\end{tabular}

a spectral energy distribution (SED) of the centre. We note that we can fit an SED for this source, as the emission in all of the Herschel bands observed at the location of the central radio core appear to be associated with the same source.

Photometry of the compact source from 3.6 to $350 \mu \mathrm{m}$ (Fig. 14) was derived using a circular aperture with diameter 1.09 arcmin centred at $\alpha=17^{\mathrm{h}} 22^{\mathrm{m}} 24.8^{\mathrm{s}}, \delta=-36^{\circ} 11^{\prime} 06^{\prime \prime}$. In order to minimize the effect of the second dust source just to the east of the radio core (the red 'blob' in the bottom right-hand panel of Fig. 14), the background emission was derived using an annulus centred at the same co-ordinate with diameter 2.84 arcmin and width 1.00 arcmin. Numerous point sources in the Spitzer bands were masked out and corrections were applied to the IRAC bands to account for the extended diffuse emission seen in the images following the instructions in the Spitzer IRAC handbook. ${ }^{5}$ The Spitzer IRAC fluxes were dereddened following the reddening law of Indebetouw et al. (2005) where $\mathrm{A}_{\mathrm{K}_{\mathrm{S}}}=2.5$ mag. ${ }^{6}$ Errors in the fluxes were determined by combining calibration errors with the error determined in the background level. The photometry is listed in Table 4 alongside the VLA photometry from Becker \& Helfand (1988), and the SED is shown in Fig. 23. The synchrotron power law for the compact radio core is shown with slope +0.27 (Becker $\&$ Helfand 1988). Following C19, we fit the SED with two modified blackbodies to derive a cool dust temperature and a dust mass. We have assumed that the dust emissivity index $\beta$ is kept constant at a value of 1.9. We find a best-fitting $\left(\chi^{2}\right)$ model with temperature $45.8 \mathrm{~K}$ (see also Fig. 21 ) and dust mass $0.18 \mathrm{M}_{\odot}(d / 11 \mathrm{kpc})^{-2}$ (where $d$ is the distance from Dubner et al. 1993). We also run a Monte Carlo analysis by perturbing the observed fluxes within their uncertainties 1000 times and refitting the SEDs. The median SED fit from this analysis produces a cold dust component with $T_{\mathrm{d}}=31.9 \pm 1.5 \mathrm{~K}$ and $M_{\mathrm{d}}=1.1 \pm 0.3 \mathrm{M}_{\odot}(d / 11 \mathrm{kpc})^{-2}$, and a hot dust component with $T_{\mathrm{d}}=242.1 \pm 6.8 \mathrm{~K}$ and $M_{\mathrm{d}}=$ $(3.9 \pm 0.4) \times 10^{-6} \mathrm{M}_{\odot}(d / 11 \mathrm{kpc})^{-2}$.

One potential issue is whether the compact source is a $\mathrm{H}$ II region instead of a SNR. Although the dust temperature we derive is hotter than the average values observed in H II regions (typically 15$30 \mathrm{~K}$; Anderson et al. 2012a), we note that some $\mathrm{H}$ II have observed dust temperatures up to $40 \mathrm{~K}$ (Povich et al. 2007; Anderson et al.

\footnotetext{
${ }^{5}$ https://irsa.ipac.caltech.edu/data/SPITZER/docs/irac/iracinstrumenthandb ook/29/

${ }^{6}$ https://irsa.ipac.caltech.edu/workspace/TMP_wYHAkz_6484/DUST/17_2 2_27_-36_11_00.v0001/extinction.html
}

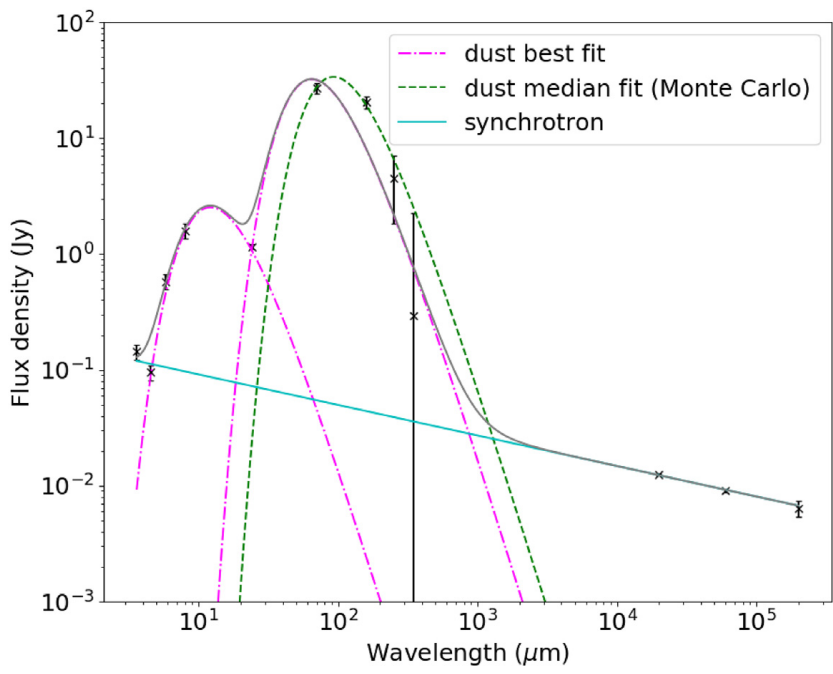

Figure 23. Spectral energy distibution of G351.2+0.1 from NIR-radio wavelengths. Fluxes are listed in Table 4. The synchrotron emission assuming a power-law slope of +0.27 (Becker $\&$ Helfand 1988) extrapolating from the VLA radio fluxes of the compact core is shown by the solid (blue) line. The best-fitting $\left(\chi^{2}\right)$ hot and cool modified blackbody fits to the photometry are shown by the dot-dashed curves (magenta) and the combination of all emission sources is shown in the solid (grey) curve. The dotted (green) curve is the resultant median modified blackbody fit from 1000 SEDs derived by normally distributing the original observed fluxes within their errors.

2012b). We next compare NIR - radio colours with those observed in $\mathrm{H}$ II regions since different emission mechanisms should result in different predicted colours in the NIR - FIR and FIR-radio. Reach et al. (2006) suggests that the Spitzer IRAC colours $(3.8-8 \mu \mathrm{m})$ can be used as a diagnostic between SNRs, the ISM (H II regions or their associated photodissociation regions, PDRs), shocked molecular gas, or ionized gas as well as synchrotron sources. Recent Spitzer and Herschel studies of $\mathrm{H}$ II regions suggest NIR - FIR colour plots can also be used as a diagnostic for their identification (Pinheiro Goncalves et al. 2011; Anderson et al. 2012a,b; Paladini et al. 2012). Similarly, the $8 \mu \mathrm{m}$ and $843 \mathrm{MHz}$ ratios can offer an alternative diagnostic (Cohen et al. 2007). Next we test whether the fluxes derived for G351.2+0.1 can reveal whether we are observing dust from an unrelated $\mathrm{H}$ II region or a SNR.

Table 5 shows various colours predicted or observed in $\mathrm{H}$ II regions in comparison to those measured for G351.2+0.1. At face value, the IR and radio colours show this source is entirely consistent with interstellar material. However there are some caveats with this. Although the IRAC colours for this source are consistent with the ISM regions in Reach et al. (2005) (see also Table 5), they are similar to that observed in the PWNe G21.5-0.9 (Zajczyk et al. 2012). Comparing with the $\log \left(F_{8} / F_{24}\right)$ versus $\log \left(F_{70} / F_{24}\right)$ colour plot of Pinheiro Goncalves et al. (2011) (their fig. 3), G351.2 + 0.1 lies along the slope of colours observed in Galactic SNRs, consistent with dust emission with a colour temperature $\left(T_{24 / 70}\right)$ of $\sim 45 \mathrm{~K}$ for $\beta=2$, and well above the different trend line observed for $\mathrm{H}$ II regions. Although the colours are consistent with the narrow range of observed IR-FIR values found in H II regions (Paladini et al. 2012), these overlap considerably with observed values for SNRs which are observed to have much wider range of colours due to the variety of morphologies, ages, emission mechanisms in the latter. Comparing with colours for other known dusty PWNe e.g. G54.1+0.3 and the Crab Nebula (see Table 5), although G54.1 satisfies none of the HII diagnostic tests, the Crab does satisfy 
Table 5. Summary of the various colour tests to resolve whether the dust source associated with the radio source observed in G351.2+0.1 originates from a H II region or a SNR. Unless specified, all the subscripts refer to the wavelength in microns.

\begin{tabular}{|c|c|c|c|c|c|c|c|}
\hline \multirow[t]{2}{*}{ Colour } & \multirow[t]{2}{*}{ H II region test } & \multicolumn{2}{|c|}{$\mathrm{G} 351.2+0.1$} & \multicolumn{2}{|c|}{ G54.1+0.3 } & \multicolumn{2}{|c|}{ Crab } \\
\hline & & Value & Consistent with H II? & Value & Н II? & Value & H II? \\
\hline$\frac{3.6}{8}\left|\frac{4.5}{8}\right| \frac{5.8}{8} \mid I_{8}{ }^{a}$ & $0.04|0.05| 0.35 \mid 1$ & $0.09|0.06| 0.36 \mid 1$ & $\checkmark$ &.. &.. & .. & .. \\
\hline $\log \left(F_{160} / F_{24}\right)^{b}$ & $>0.8$ & 1.24 & $\checkmark$ & 0.10 & $x$ & 0.45 & $\times$ \\
\hline $\log \left(F_{160} / F_{70}\right)^{b}$ & $>-0.2$ & -0.12 & $\checkmark$ & -0.5 & $x$ & -0.12 & \\
\hline $\log \left(F_{70} / F_{24}\right)^{b}$ & $>0.8$ & 1.37 & $\checkmark$ & 0.6 & $x$ & 0.58 & $x$ \\
\hline $\log \left(F_{24} / F_{8}\right)^{b}$ & $<1.0$ & -0.14 & $\checkmark$ & 1.59 & $x$ & 0.52 & \\
\hline $\log \left(F_{24} / F_{70}\right)^{c}$ & -1.5 to -1.0 & -1.37 & $\checkmark$ & -0.58 & $x$ & -0.12 & $x$ \\
\hline$F_{8} / S_{843 \mathrm{MHz}}{ }^{d}$ & $27 \pm 10$ & 282 & $?$ & 1.4 & $x$ & 0.02 & $x$ \\
\hline
\end{tabular}

References are ${ }^{a}$ Reach et al. (2006), ${ }^{b}$ Anderson et al. (2012a), ${ }^{c}$ Paladini et al. (2012), ${ }^{d}$ Cohen et al. (2007). Also shown are the results of the same tests for the PWNe G54.1+0.3 (fluxes are taken from Temim et al. 2017 and Rho et al. 2018) and the Crab Nebula (from De Looze et al. 2019).

some. It is not clear how the presence of ejecta dust in SNe would affect these tests, and whether, given the wide range of properties of SNRs, it is even possible to use colours to distinguish between source types. We conclude therefore that we cannot rule out that the dust observed in the centre of G351.2 is unrelated interstellar material, but nor can we rule out an $\mathrm{SN}$ ejecta origin.

\section{DISCUSSION}

\subsection{Types of sources detected}

Here we discuss the detection rates found in our Herschel study. This work finds signatures of dust in 10 (8 percent) SNRs in the Galactic Plane (detection level of 1) compared to the 29 detected in C19 (41 per cent detection rate). In total, we estimate a FIR detection rate of 21 per cent of Galactic SNRs with $|b| \leq 1^{\circ}$ and classed as a level 1 detection. Table 6 and Fig. 24 give a summary of the types of SNRs detected in this study. Of our new FIR detections, we observe dust emission from the shell/outer shock region of eight SNRs, and from the inner ejecta region (interior to the reverse shock) of four sources. We detect 1 Type Ia and 1 core-collapse SNe. Combining with $\mathrm{C} 19$, this gives Herschel-detected SN dust structures in 13 core-collapse $\mathrm{SNe}$, including 4 confirmed $\mathrm{PWNe}$, and 2 Type Ia's in the Hi-GAL survey. Including the other Herschel discovered dusty remnants in the Milky Way that were not covered by the Hi-GAL survey, that is Cas A, the Crab Nebula, Tycho, Kepler, and G292.0+1.8 (hereafter, we name this group the historical dusty SNRs), the final statistics for dust features related to $\mathrm{SN}$ structures in the Galaxy are therefore 16 core collapse SNe, including 6 PWNe, and 4 Type Ias (Table 6). Based on these 20 sources where the $\mathrm{SN}$ explosion type is known, our dust-detected sample is made up of 80 per cent core-collapse SNRs, and 20 percent Ia SNRs, closely mirroring the observed rates of core-collapse and Type Ia SN explosions (van den Bergh 1993; The et al. 2006) with the former occurring at rates of $\sim 2-3$ SNe per century (van den Bergh 1991; Dragicevich, Blair \& Burman 1999; Diehl et al. 2006; Li et al. 2011).

The detection of SN-related dust features in the Herschel images of the Type Ias G306.3-0.9 (this work) and G344.7-0.1 (C19) could provide further insight into dust formation in Ia SNRs, for which there is not yet any evidence of ejecta dust forming in their remnants. Although at first glance, the IR emission seen in the Herschel images appears to originate from reserve shock-heated material, we saw in Section 2.1 that for G306.3-0.9, the dust appears to be associated with material swept up by the blast wave, or unrelated interstellar clouds, and not from supernova dust. A similar conclusion was reached by Gomez et al. (2012a) for the Type Ia SNRs Kepler and Tycho.

Compared with previous FIR surveys of Galactic SNRs by Arendt (1989) and Saken et al. (1992) our higher resolution helps us to better distinguish SNRs from the surrounding ISM, giving us higher detections rates within the Galactic Plane. In comparison with Saken et al. (1992), we detect seven sources in common $(\mathrm{G} 31.9+0.0$, G33.2-0.6, G43.3-0.2, G54.1+0.3, $\mathrm{G} 304.6+0.1, \mathrm{G} 340.6+0.3$, and $\mathrm{G} 349.7+0.2)$ and upgrade 16 of their SNRs to level 1 Herschel detections from the equivalent of their IRAS levels 2, 3, and 4. We also find nine detections in common with Arendt (1989; G11.2-0.3, G31.9+0.9, G34.7-0.4, G43.3-0.2, G54.1+0.3, G304.6+0.1, G340.6+0.3, G348.7+0.3, and $\mathrm{G} 349.7+0.2$ ) and upgrade 15 sources to level 1 Herschel detections from their IRAS levels 2, 3, and 4. Although there are a small number of detections in each study which, with Herschel, we downgrade from a level 1 detection ( 8 from Saken et al. 1992 and 2 from Arendt 1989), we increase the detection rate of Galactic SNRs within each sample from 17 to 24 per cent for the Saken et al. (1992) sample, and from 13 to 26 percent for the Arendt (1989) sample (excluding sources which were not included in the Green 2014 catalogue).

An interesting question is whether there exists a trend with how dusty a SNR is and its age (e.g. Otsuka et al. 2010; Gall et al. 2014). (Ages are listed in Table A1 where known, see references therein.) Not including those without estimated ages, Fig. 24 shows that the highest proportion of our detected sources are young, $\leq 5 \mathrm{kyr}$, which may be because the SNR is still compact so that surface brightness is higher and therefore more easily 'seen' in the comparison of the multicolour Herschel images. The historical dusty SNRs also lie within this age range (Fig. 24); indeed these are mostly clustered towards younger ages than the majority of the det=1 SNRs in our Hi-GAL survey (where the youngest det $=1$ SNR is the core-collapse SNR G29.7-0.3, Kes 75). This suggests that the previous historical dusty SNRs were biased to the youngest (and potentially dustiest) objects, whereas the sample in this work has detected dust signatures associated with SNRs at a wider range of ages up to $10^{2} \mathrm{kyr}$.

However, for all SN ages and types we are more likely to find level 3 detections, where there is only unrelated FIR emission in the region. Although this may imply that there is no dust within these SNRs, we are also greatly affected by confusion in cases where 
Table 6. Summary of the level 1 detected sample in this work and C19. We also include the previously detected Herschel Galactic SNRs as 'historical remnants': Tycho, Kepler, Cas A, the Crab Nebula, and G292.0+1.8.

\begin{tabular}{lcccc}
\hline & Detection type & \multicolumn{3}{c}{ Number detected } \\
& & C19 & This work & Historical \\
\hline SNR Region $\sharp$ & Shell / outer shock region & 23 & 8 & 3 \\
& Inner ejecta region * & 8 & 4 & 3 \\
& Confirmed PWN $\dagger$ & 4 & 0 & 1 \\
Age (kyr) & Interacting molecular cloud & 0 & 1 & - \\
& $\leq 1$ & 2 & 1 & 3 \\
& $1<$ Age $\leq 10$ & 12 & 2 & 2 \\
SN Type & $10<$ Age $\leq 20$ & 1 & 0 & - \\
& Unknown & 4 & 0 & - \\
& Type Ia & 1 & 7 & - \\
& Core collapse & 12 & 1 & 2 \\
& Unknown & 16 & 8 & 3 \\
\hline
\end{tabular}

*Sources from which we detect FIR emission from the inner region are: G11.2-0.3, G16.4-0.5, G21.5-0.9, G21.5-0.1, G29.7-0.3, G34.7-0.4, G54.1+0.3, and G344.7-0.1 (C19), and G306.3-0.9, G350.1-0.3, G351.2+0.1, and G357.7-0.1 (this work). $\dagger$ SNRs for which there is evidence that the detected central region is associated with the confirmed PWN. We note that G351.2+0.1 could be associated with a PWN but the compact object has radio, NIR - FIR detection only and other sources cannot be ruled out (see Section 2.1).

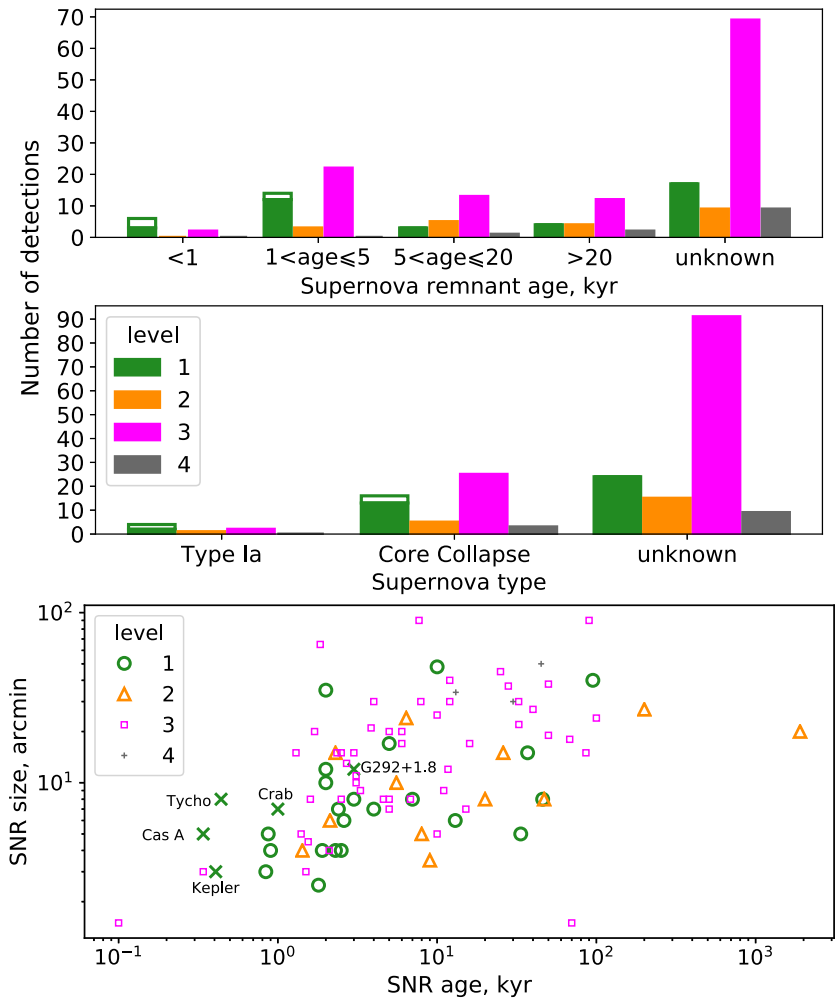

Figure 24. As in C19, we show a summary of the source types detected in the sample, including those in C19. Top: Filled histograms compare the number of sources with different ages for a given detection classification in Hi-GAL. The unfilled bar includes the previous Herschel detections of Galactic SNRs not covered by the Hi-GAL survey: Cas A, the Crab Nebula, Tycho, Kepler, and G292.0+1.8. Middle: As top panel, but showing the number of sources with different SN types for a given detection classification. Bottom: The detection level compared with the size and age of SNR; the crosses indicate the properties of the previous historical SNRs. The age is unknown for 104 SNRs from our sample. sources may have a similar dust temperature to ISM dust or already starting to mix with the ISM (i.e. larger/older), wherein it becomes more difficult to separate ejecta and unrelated material. We therefore expect that the detection levels quoted here are a lower limit on the number of SNRs within the Galactic Plane which contain dust.

Fig. 25 shows the location of all of the SNRs studied within the Galactic Plane with Herschel as part of the Hi-GAL survey. We find that the majority of the SNRs with clear dust signatures (detection level $=1$ ) are located towards the central regions of the Galactic Plane in both longitude and latitude. This distribution follows the number density of SNRs in general, which are concentrated towards low latitudes and in the inner regions of the Galaxy (Green 2014, Fig. 25, top panel).

The distribution of the 'dust-detected' sources in this work can be explained due to our sample being dominated by the remnants of core-collapse SNe, which make up the majority of SNRs in the Galaxy. Core-collapse remnants are observed to be found at lower latitudes, closely linked to star forming regions concentrated in the thin disc of the Galaxy (Hakobyan et al. 2016). Instead, Type Ia's, which constitute only a small fraction of our det $=1$ sample with known SN types, are observed to lie at a wider range of latitudes, with Galactic scale heights of $\sim 2$ times that of corecollapse remnants (Hakobyan et al. 2017) due to their association with the older stellar population. We may therefore be biased against finding dust in Type Ia SNRs in this work.

In order to interpret the distribution of dusty SNRs in the Galaxy, we need to first note our selection effects. Although we start from a blind Herschel survey of the sky with Hi-GAL, and as such the dust information is 'complete' within $b \pm 1^{\circ}$, due to the high levels of confusion in the FIR we have to rely on the known locations of SNRs, where we have used the Green (2004) catalogue. Since this is the fundamental selection effect for this work, here we briefly mention its completeness. Green (2015) comments that the Galactic SNR catalogue is likely missing intrinsically faint radio SNRs, SNRs where the physical size is small (diameters $<1.5$ arcmin), and more distant SNRs with smaller angular sizes, as well as 


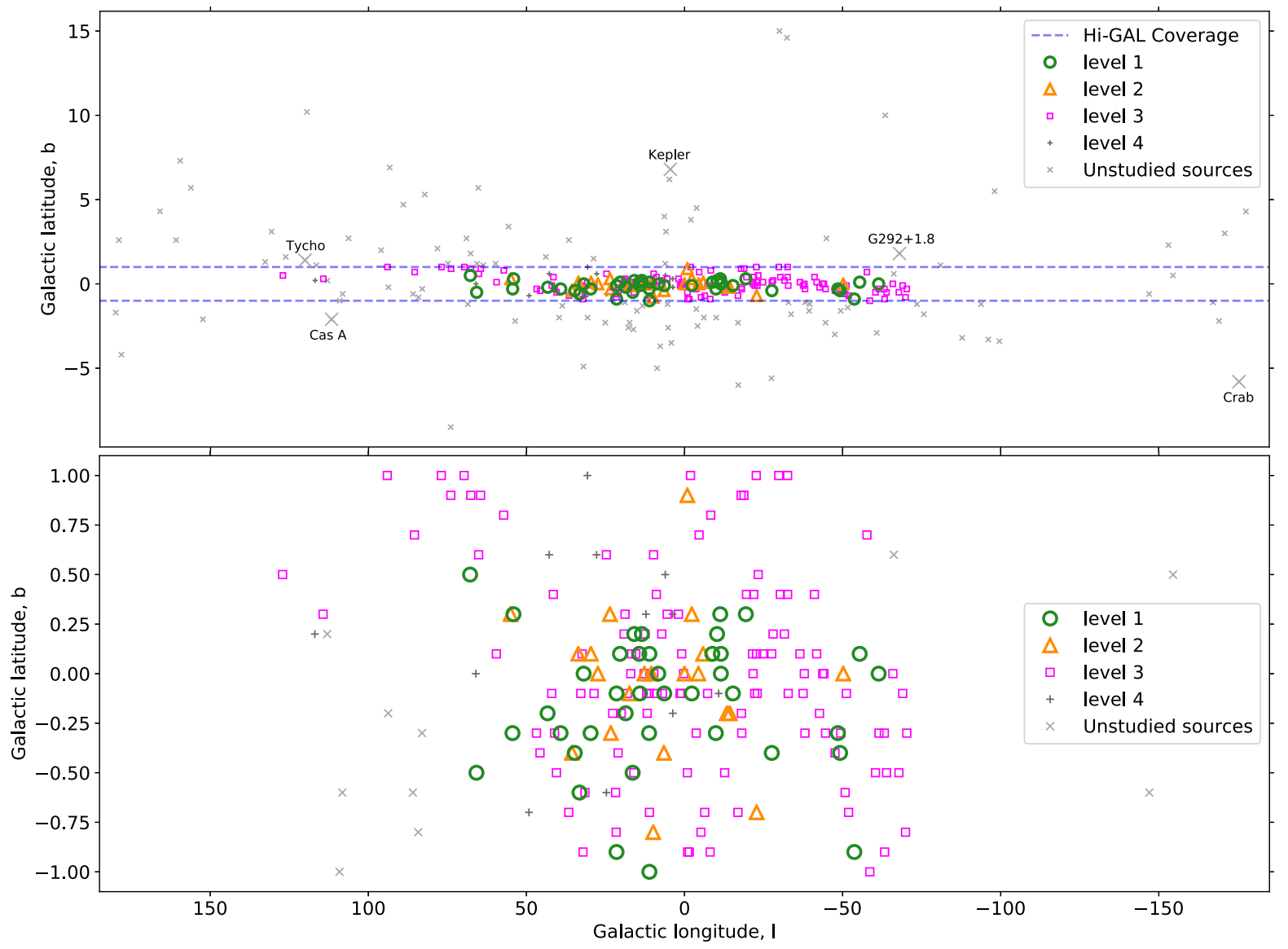

Figure 25. Location of Galactic SNRs from Green's catalogue as a function of their assigned detection number, 1-4 (green circles, orange triangles, magenta squares, and grey plus signs, respectively) and those not covered in the Hi-GAL survey (i.e. not studied here) shown by the grey crosses. The dashed blue lines indicate the extent of the area surveyed by Hi-GAL. Top: All SNRs from Green's catalogue. Bottom: Sources within the Galactic Plane with $|b| \leq 1$ (including Chawner et al. 2019). Hi-GAL data are unavailable for 10 sources within the Galactic Plane.

many sources having uncertain distances due to the use of the $\Sigma-D$ relation. Therefore our sample will also suffer from the same selection effects. We note that our det $=1$ sampled follows the same $l, b$ distribution as their radio-bright SNR subsample (with a surface brightness cut $\left.(1 \mathrm{GHz})>10^{-20} \mathrm{~W} \mathrm{~m}^{-2} \mathrm{~Hz}\right)$ which they argue suffers from fewer selection effects than the whole Galactic radio SNR catalogue. However, the overlap between our detections and the bright SNR sample can be attributed to the fact that we rely on the radio images (where available) to identify $\mathrm{SN}$-related dust features.

We include the historical dusty SNRs for reference in Fig. 25. These lie outside the inner Galactic Plane, and yet still have associated dust features (and indeed a significant amount of ejecta dust). Again this implies that the detection levels quoted here are likely lower limits, since there are $\sim 100$ more SNRs in the catalogue of Green (2014) not included in the region surveyed by Hi-GAL (Fig. 25, top panel).

Our previous study (C19) covered $10 \leq|l| \leq 60^{\circ}$. Although this study covers the entire Galactic longitude range, the majority of additional sources are within $|l| \leq 10^{\circ}$. This region is heavily contaminated with ISM dust, resulting in a lower detection rate in this study compared with C19. An additional factor causing a lower detection rate in this work compared to C19 could be the lack of Spitzer data in some regions; in particular Spitzer MIPS $24 \mu \mathrm{m}$ has better spatial resolution than Herschel, which helps to identify warm
SN dust structures more confidently. In our study, the majority of the SNRs are classified as 'heavily confused' in these regions. We remind the reader that the requirement of ancillary X-ray, radio, or optical data in order to identify SN features against the surrounding ISM/CSM, and the non-uniformity of the availability and quality of this data may have further resulted in a bias in our Hi-GAL Galactic Plane SNR sample.

In summary, we are more likely to detect dust in remnants where we have good quality ancillary radio, X-ray, optical or Spitzer $24 \mu \mathrm{m}$ images, that are located at low galactic latitudes due to both the higher concentration of core-collapse SNRs and bright radio SNRs in these regions, with PWNe due to the additional heating source that raises the $\mathrm{SN}$ dust temperatures above the ambient ISM level (similarly for shock heated dusty shells).

\subsection{Interpreting the SNR dust temperatures: is warm dust in SNRs collisionally heated?}

In Section 3.3 we identified warm dust in shocked regions of X-ray and radio emission, suggesting that these interactions are heating this material. We compare models of shock heating to determine the gas properties required to heat the dust to the temperatures seen in Fig. 21. In the case of collisional dust heating, the dust temperature in X-ray emitting plasmas can be given by the following expression 


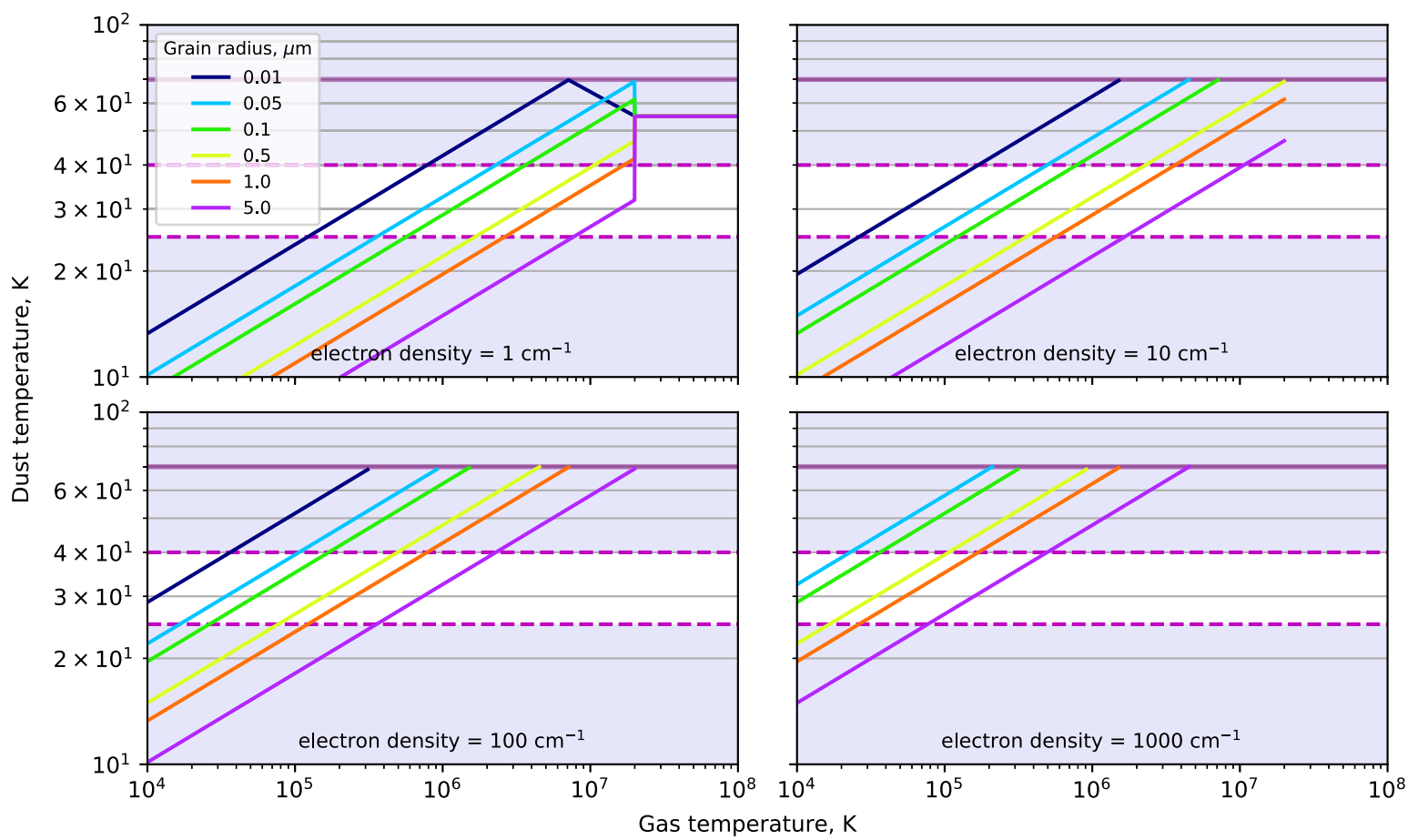

Figure 26. Temperature of collisionally heated dust given by equation (2) for electron densities of forward shock regions and molecular clouds assuming graphite and silicate dust grains. The white region between the dashed magenta lines gives the range of temperatures observed in our SNRs in Fig. 21 . The solid purple line is at $70 \mathrm{~K}$, above which this relation for collisional heating no longer applies (hence the lines end at $T_{d}=70 \mathrm{~K}$ ).

for $T_{\mathrm{d}} \lesssim 70 \mathrm{~K}$ (Dwek 1987):

$T_{\mathrm{d}}^{4}=\frac{\left(\frac{32}{\pi m_{e}}\right)^{0.5} n_{e}\left(k T_{\mathrm{gas}}\right)^{1.5} h\left(a, T_{\mathrm{gas}}\right)}{4 \sigma<Q\left(a, T_{\mathrm{d}}\right)>}$,

where $n_{e}$ is the electron density $\left(\mathrm{cm}^{-3}\right), a$ is the grain radius $(\mu \mathrm{m}), T_{\text {gas }}$ is the gas temperature $(\mathrm{K}), h\left(a, T_{\text {gas }}\right)$ is the grain heating efficiency $(\sim 1$ for most grain sizes), $<Q>$ is the Planck averaged dust absorption coefficient and we assume $<Q\left(a, T_{\mathrm{d}}\right)>=0.16 a T_{\mathrm{d}}^{1.94}$ for graphite and silicates (Dwek \& Arendt 1992). As indicated by Dwek (1987) the dust temperature provides useful constraints on the allowable combinations of gas density and temperature behind the shock.

In Fig. 26 we consider three cases of electron density similar to that observed in shocked regions of CCSNRs (e.g. for Cas A $n_{e}=16 \mathrm{~cm}^{-3}$ and $n_{e}=61 \mathrm{~cm}^{-3}$ in the hot and cold components, respectively; Willingale et al. 2003), and one case of a typical molecular cloud density $\left(n_{e} \sim 1000 \mathrm{~cm}^{-3}\right)$. If the SNR dust in Fig. 21 is collisionally heated we can rule out small grains $(a \lesssim$ $0.1 \mu \mathrm{m}$ ) for all densities other than $1 \mathrm{~cm}^{-3}$. This would require unexpectedly cool electron gas to give the range of dust temperatures $(\sim 25-40 \mathrm{~K})$ where $\mathrm{X}$-ray measurement suggest that typical shocked gas (or electron) temperatures are $\gtrsim 10^{6} \mathrm{~K}$ (e.g. Koo et al. 2016). For our lowest density $\left(n_{e}=1 \mathrm{~cm}^{-3}\right)$ the full range of SNR temperatures is available to us with grains of radius $0.5,1.0$, or $5.0 \mu \mathrm{m}$ at reasonable gas temperatures of $T_{g}>10^{6}$. Smaller grains could provide the observed results, although this is less likely as the gas temperature would be lower than expected for shocked gas. For the densest region we find that the surrounding gas must be cool $\left(T_{g} \lesssim 10^{5} \mathrm{~K}\right)$ and we must have relatively large dust grains $(a>1 \mu \mathrm{m})$ : it is unlikely that this describes our SNRs. It is possible that these emitting dust grains are located in post-shock regions.

\section{CONCLUSIONS}

We searched for FIR counterparts of known supernova remnants (SNRs) in the entire Galactic Plane, as surveyed by Herschel at 70 $500 \mu \mathrm{m}$, to supplement the first-look catalogue of 71 SNRs studied by Chawner et al. (2019). Of 119 sources studied here, we find that 10 (8 per cent) have a clear FIR detection of dust associated with the SNR. When combined with $\mathrm{C} 19$, this gives a total of 39 FIR detected sources out of 190 known remnants with $|b| \leq 1^{\circ}$ across the entire Galactic Plane (a detection rate of 21 per cent): with dust signatures detected in the remnants of 13 core-collapse supernovae (SNe), including 4 Pulsar Wind Nebulae (PWNe), and 2 Type Ia $\mathrm{SNe}$. A further 24 are detected in sources with unknown types.

Additionally we have shown that:

(i) We tend to detect dust in younger SNRs.

(ii) We confirm the detection of ejecta dust within the corecollapse SNR G350.1-0.3, as seen in Spitzer $24 \mu \mathrm{m}$ and now Herschel $70 \mu \mathrm{m}$ images. This adds to a sample of only $\sim 10 \mathrm{SNRs}$ from which ejecta dust has been observed and indicates that $\mathrm{SNe}$ can form dust grains from elements synthesized by the SN explosion. We see no evidence of cooler dust in the ejecta nor dust located at the location of the compact central object.

(iii) We suggest that the dust features previously proposed to be related to the G357.7+0.3 SNR (seen previously with Spitzer) are dust bubbles associated with young star forming regions, and not with the SNR itself.

(iv) We reveal dust associated with G351.2+0.1. We propose that the FIR emission from the latter source originates from a PWN (though we cannot conclusively rule out a $\mathrm{H}$ II region). We estimate a dust temperature and dust mass for $\mathrm{G} 351.2+0.1$ of $45.8 \mathrm{~K}$ and $M_{\mathrm{d}}=0.18 \mathrm{M}_{\odot}$. 
(v) We identify warm dust in several SNRs with temperatures between $\sim 25$ and $40 \mathrm{~K}$ which, if collisionally heated, indicates that there must be relatively cool shocked plasma, or the dust must be made up of large grains $(a>1 \mu \mathrm{m})$. However, we caution that there could still be contamination in our apertures with unrelated dust along the line of sight, which we have shown could result in dust temperatures that are biased low. We see that the largest source of uncertainty in a FIR catalogue of SNRs is the result of significant confusion with unrelated dust in the Milky Way.

(vi) We estimate that six of our sample contain considerable masses of dust, although ISM contamination may bias our dust masses high.

\section{ACKNOWLEDGEMENTS}

We are grateful to Aya Bamba, and Hiroya Yamuguchi for providing X-ray data for G298.6-0.0 and G344.7-0.1 respectively, also to Ryan Lau and Matthew Hankins for providing SOFIA data for Sgr A East. We thank Felix Priestley for informative discussions on this topic. We also thank the anonymous referee for their contribution to the paper.

$\mathrm{HC}$, HLG, and PC acknowledge support from the European Research Council (ERC) in the form of Consolidator Grant COSMICDUST (ERC-2014-CoG-647939). MJB acknowledges support from the ERC in the form of Advanced Grant SNDUST (ERC-2015AdG-694520). MM acknowledges support from an STFC Ernest Rutherford fellowship (ST/L003597/1). Herschel is an ESA space observatory with science instruments provided by European-led Principal Investigator consortia and with important participation from NASA. IDL gratefully acknowledges the support of the Research Foundation Flanders (FWO).

This research has made use of data from the HiGAL survey (2012hers.prop.2454M, 2011hers.prop.1899M, 2010hers.prop.1172M, 2010hers.prop.358M) and ASTROPY, ${ }^{7}$ a community-developed core PYTHON package for Astronomy (The Astropy Collaboration 2013, 2018). This research has made use of the NASA/ IPAC Infrared Science Archive, which is operated by the Jet Propulsion Laboratory, California Institute of Technology, under contract with the National Aeronautics and Space Administration. The scientific results reported in this article are based in part on data obtained from the Chandra Data Archive.

\section{REFERENCES}

Acero F., Renaud M., Ballet J., Hewitt J. W., Rousseau R., Tanaka T., 2015, A\&A, 580, A74

Andersen M., Rho J., Reach W. T., Hewitt J. W., Bernard J. P., 2011, ApJ, 742,7

Anderson L. D., Bania T. M., Balser D. S., Rood R. T., 2011, ApJS, 194, 32 Anderson L. D., Zavagno A., Barlow M. J., García-Lario P., Noriega-Crespo A., 2012a, A\&A, 537, A1

Anderson L. D. et al., 2012b, A\&A, 542, A10

Anderson L. D. et al., 2017, A\&A, 605, A58

Arendt R. G., 1989, ApJS, 70, 181

Arikawa Y., Tatematsu K., Sekimoto Y., Takahashi T., 1999, PASJ, 51, L7

Arzoumanian Z., Gotthelf E. V., Ransom S. M., Safi-Harb S., Kothes R., Landecker T. L., 2011, ApJ, 739, 39

Bamba A., Ueno M., Koyama K., Yamauchi S., 2001, PASJ, 53, 21

Bamba A., Sawada M., Nakano Y., Terada Y., Hewitt J., Petre R., Angelini L., 2016, PASJ, 68, S51

${ }^{7}$ http://www.astropy.org
Barlow M. J. et al., 2010, A\&A, 518, L138

Beaumont C. N., Williams J. P., Goodman A. A., 2011, ApJ, 741, 14

Becker R. H., Helfand D. J., 1988, AJ, 95, 883

Bietenholz M. F., Bartel N., 2008, MNRAS, 386, 1411

Bietenholz M. F., Matheson H., Safi-Harb S., Brogan C., Bartel N., 2011, MNRAS, 412, 1221

Billot N., Noriega-Crespo A., Carey S., Guieu S., Shenoy S., Paladini R., Latter W., 2010, ApJ, 712, 797

Bocchino F., van der Swaluw E., Chevalier R., Bandiera R., 2005, A\&A, 442,539

Bocchino F., Bandiera R., Gelfand J., 2010, A\&A, 71, 1

Borkowski K. J., Reynolds S. P., Green D. A., Hwang U., Petre R., Krishnamurthy K., Willett R., 2014, ApJ, 790, 18

Borkowski K. J., Reynolds S. P., Roberts M. S. E., 2016, ApJ, 819, 160

Brogan C., Goss W., 2003, AJ, 125, 272

Brogan C. L., Gaensler B. M., Gelfand J. D., Lazendic J. S., Lazio T. J. W., Kassim N. E., McClure-Griffiths N. M., 2005, ApJ, 629, L105

Brogan C. L., Gelfand J. D., Gaensler B. M., Kassim N. E., Lazio T. J., 2006, ApJ, 639, 5

Burton M. G., Lazendic J. S., Yusef-Zadeh F., Wardle M., 2004, MNRAS, 348,638

Camilo F., Ransom S. M., Gaensler B. M., Lorimer D. R., 2009a, ApJ, 700, 34

Camilo F., Ng C. Y., Gaensler B. M., Ransom S. M., Chatterjee S., Reynolds J., Sarkissian J., 2009b, ApJ, 703, 55

Carlton A. K., Borkowski K. J., Reynolds S. P., Hwang U., Petre R., Green D. A., Krishnamurthy K., Willett R., 2011, ApJ, 737, L22

Case G. L., Bhattacharya D., 1998, ApJ, 504, 761

Castelletti G., Supan L., Dubner G., Joshi B. C., Surnis M. P., 2013, A\&A, 557, L15

Castro D., Slane P. O., Gaensler B. M., Hughes J. P., Patnaude D. J., 2011, ApJ, 734, 86

Castro D., Slane P., Carlton A., Figueroa-Feliciano E., 2013, ApJ, 774, 36

Caswell J. L., Murray J. D., Roger R. S., Cole D. J., Cooke D. J., 1975, A\&A, 45, 239

Caswell J. L., Haynes R. F., Milne D. K., Wellington K. J., 1980, MNRAS, 190,881

Caswell J. L., Haynes R. F., Milne D. K., Wellington K. J., Smith R. M., 1983a, Publ. Astron. Soc. Aust., 5, 227

Caswell J. L., Haynes R. F., Milne D. K., Wellington K. J., 1983b, MNRAS, 203, 595

Caswell J. L., Kesteven M. J., Stewart R. T., Milne D. K., Haynes R. F., 1992, ApJ, 399, L151

Chawner H. et al., 2019, MNRAS, 483, 70 (C19)

Chen Y., Su Y., Slane P. O., Wang Q. D., 2004, ApJ, 616, 27

Chevalier R. A., 2005, ApJ, 619, 839

Cohen M. et al., 2007, MNRAS, 374, 979

Combi J. A., Benaglia P., Romero G. E., Sugizaki M., 2005, A\&A, 431, L9

Combi J. A., Albacete Colombo J. F., Martí J., 2008, A\&A, 28, L25

Combi J. A. et al., 2010a, A\&A, 522, A50

Combi J. A. et al., 2010b, A\&A, 523, A76

Combi J. A., García F., Suárez A. E., Luque-Escamilla P. L., Paron S., Miceli M., 2016, A\&A, 592, 8

De Looze I., Barlow M. J., Swinyard B. M., Rho J., Gomez H. L., Matsuura M., Wesson R., 2017, MNRAS, 465, 3309

De Looze I. et al., 2019, MNRAS, 488, 164

De Vis P. et al., 2017, MNRAS, 471, 1743

Diehl R. et al., 2006, Nature, 439, 45

Doherty M., Johnston S., Greeb A. J., Roberts M. S., Romani R. W., Gaensler B. M., Crawford F., 2003, MNRAS, 339, 1048

Downes A. J. B., Pauls T., Salter C. J., 1980, A\&A, 92, 46

Dragicevich P. M., Blair D. G., Burman R. R., 1999, MNRAS, 302, 693

Dubner G. M., Moffett D. A., Goss W. M., Winkler P. F., 1993, AJ, 105, 2251

Dubner G., Giacani E., Reynoso E., Goss W. M., Roth M., Green A., 1999, AJ, 118, 930

Dubner G., Giacani E., Reynoso E., Parón S., 2004, A\&A, 426, 201 
Dunne L., Eales S., Ivison R., Morgan H., Edmunds M., 2003, Nature, 424, 285

Dunne L. et al., 2009, MNRAS, 394, 1307

Dwek E., 1987, ApJ, 322, 812

Dwek E., Arendt R. G., 1992, ARA\&A, 30, 11

Dwek E., Galliano F., Jones A. P., 2007, ApJ, 662, 927

Folgheraiter E. L., Warwick R. S., Watson M. G., Koyama K., 1997, MNRAS, 292, 365

Frail D. A., Goss W. M., Whiteoak J. B. Z., 1994, AJ, 437, 23

Frail D. A., Goss W. M., Reynoso E. M., Giacani E. B., Green A. J., Otrupcek R., 1996, AJ, 111, 1651

Frank K. A., Burrows D. N., Park S., 2015, ApJ, 810, 113

Froebrich D. et al., 2015, MNRAS, 454, 2586

Gaensler B. M. et al., 2008, ApJ, 680, L37

Gaensler B. M., Manchester R. N., Green A. J., 1998, MNRAS, 296, 813

Gaensler B. M., Gotthelf E. V., Vasisht G., 1999, ApJ, 526, L37

Gaensler B. M., Fogel J. K. J., Slane P. O., Miller J. M., Wijnands R., Eikenberry S. S., Lewin W. H. G., 2003, ApJ, 594, L35

Gall C., Hjorth J., Andersen A. C., 2011, A\&AR, 19, 43

Gall C. et al., 2014, Nature, 511, 326

Gao X. Y., Reich P., Hou L. G., Reich W., Han J. L., 2019, A\&A, 623, A105

Ghavamian P., Williams B. J., 2016, ApJ, 831, 188

Giacani E., Smith M. J. S., Dubner G., Loiseau N., Castelletti G., Paron S., 2009, A\&A, 507, 841

Giacani E., Smith M. J. S., Dubner G., Loiseau N., 2011, A\&A, 531, A138

Gök F., Sezer A., 2012, MNRAS, 419, 1603

Gomez H. L. et al., 2012a, MNRAS, 420, 3557

Gomez H. L. et al., 2012b, ApJ, 760, 96

Goss W. M., Schwarz U. J., van Gorkom J. H., Ekers R. D., 1985, MNRAS, 215,69

Gotthelf E. V. et al., 2014, ApJ, 788, 1

Gray A. D., 1994a, MNRAS, 270, 835

Gray A., 1994b, MNRAS, 270, 847

Green J. A. et al., 2010, MNRAS, 417, 1964

Green D. A., 2004, Bull. Astron. Soc. India, 32, 335

Green D. A., 2011, Bull. Astron. Soc. India, 39, 289

Green D. A., 2014, Bull. Astron. Soc. India, 42, 47

Green D. A., 2015, MNRAS, 454, 1517

Green A. J., Frail D. A., Goss W. M., Otrupcek R., 1997, AJ, 114, 2058

Greisen E. W., 2003, in Heck A., ed., Astrophysics and Space Science Library Vol. 285, Information Handling in Astronomy - Historical Vistas. Kluwer Academic Publishers, Dordrecht, p. 109

Hakobyan A. A. et al., 2016, MNRAS, 456, 2848

Hakobyan A. A. et al., 2017, MNRAS, 471, 1390

Halpern J. P., Gotthelf E. V., Camilo F., 2012, ApJ, 753, 10

Harrus I. M., Slane P. O., 1999, ApJ, 516, 811

Heinz S. et al., 2013, ApJ, 779, 171

Helfand D. J., Velusamy T., Becker R. H., Lockman F. J., 1989, ApJ, 341, 151

Helfand D. J., Agüeros M. A., Gotthelf E. V., 2003, ApJ, 592, 941

HESS Collaboration, 2008a, A\&A, 517, 509

HESS Collaboration, 2008b, A\&A, 836, 829

HESS Collaboration, 2011, A\&A, 531, A81

HESS Collaboration, 2014, ApJ, 794, 2

HESS Collaboration, 2015, A\&A, 574, A27

Hewitt J. W., Yusef-Zadeh F., 2009, ApJ, 694, 16

Higashi Y. et al., 2008, ApJ, 683, 957

Hurley K. et al., 1999, ApJ, 510, L111

Hwang U., Markert T. H., 1994, ApJ, 431, 819

Hwang U., Petre R., Hughes J. P., 2000, ApJ, 532, 970

Indebetouw R. et al., 2005, ApJ, 619, 931

Indebetouw R. et al., 2014, ApJ, 782, L2

Irwin M., 2010, UKIRT Newslett., 26, 14

Ishihara D. et al., 2010, A\&A, 521, L61

Jackson M. S., Safi-Harb S., Kothes R., Foster T., 2008, ApJ, 674, 936

Jeong I.-G., Koo B.-C., Cho W.-K., Kramer C., Stutzki J., Byun D.-Y., 2013, ApJ, 770, 105
Kamitsukasa F., Koyama K., Uchida H., Nakajima H., Hayashida K., Mori K., Katsuda S., Tsunemi H., 2015, PASJ, 67, 161

Kaspi V. M., Manchester R. N., Johnston S., Lyne A. G., D’Amico N., 1992, ApJ, 399, L155

Kaspi V. M., Manchester R. N., Johnston S., Lyne A. G., D’Amico N., 1996, AJ, 111, 2028

Kilpatrick C. D., Bieging J. H., Rieke G. H., 2016, ApJ, 816, 1

Klochkov D., Suleimanov V., Sasaki M., Santangelo A., 2016, A\&A, 12, 8

Koo B.-C., Moon D.-S., 1997, ApJ, 485, 263

Koo B.-C., Kim K.-T., Seward F. D., 1995, ApJ, 447, 211

Koo B.-C., Lee J.-J., Jeong I.-G., Seok J. Y., Kim H.-J., 2016, ApJ, 821, 20

Kothes R., Dougherty S. M., 2007, A\&A, 1000, 993

Kothes R., Uyaniker B., Reid R. I., 2005, A\&A, 444, 871

Krause O., Birkmann S. M., Rieke G. H., Lemke D., Klaas U., Hines D. C., Gordon K. D., 2004, Nature, 432, 596

Kumar H. S., Safi-Harb S., Slane P. O., Gotthelf E. V., 2014, ApJ, 781, 41

Lacey C. K., Lazio T. J. W., Kassim N. E., Duric N., Briggs D. S., Dyer K. K., 2001, ApJ, 559, 954

Lakićević M. et al., 2015, ApJ, 799, 50

Lau R. M., Herter T. L., Morris M. R., Li Z., Adams J. D., 2015, Science, 348,413

Lazendic J. S., Slane P. O., 2006, ApJ, 647, 350

Lazendic J. S., Slane P. O., Hughes J. P., Chen Y., Dame T. M., 2005, ApJ, 618,733

Lazendic J. S., Wardle M., Whiteoak J. B., Burton M. G., Green A. J., 2010, MNRAS, 409, 371

Leahy D. A., Green K. S., 2012, ApJ, 760, 4

Leahy D. A., Ranasinghe S., 2016, ApJ, 817, 74

Leahy D., Tian W., 2006, A\&A, 257, 251

Leahy D. A., Tian W. W., 2008, AJ, 135, 167

Leahy D. A., Naranan S., Singh K. P., 1986, MNRAS, 220, 501

Leahy D. A., Tian W., Wang Q. D., 2008, AJ, 136, 1477

Leahy D., Green K., Tian W., 2014, MNRAS, 438, 1813

Lemiere A., Slane P., Gaensler B. M., Murray S., 2009, ApJ, 706, 1269

Li W., Chornock R., Leaman J., Filippenko A. V., Poznanski D., Wang X., Ganeshalingam M., Mannucci F., 2011, MNRAS, 412, 1473

Lockman F. J., 1989, ApJS, 71, 469

Lopez L. A., Ramirez-Ruiz E., Castro D., Pearson S., 2013, ApJ, 764, 50

Lovchinsky I., Slane P., Gaensler B. M., Hughes J. P., Ng C.-Y., Lazendic J. S., Gelfand J. D., Brogan C. L., 2011, ApJ, 731, 70

Maeda Y. et al., 2002, ApJ, 570, 671

Mancini M., Schneider R., Graziani L., Valiante R., Dayal P., Maio U., Ciardi B., Hunt L. K., 2015, MNRAS, 451, L70

Mangano V., Burrows D. N., Park S., Shea T. K., 2014, in AAS/High Energy Astrophysics Division \#14. AAS/High Energy Astrophysics Division. p. 120.05

Matsuura M. et al., 2009, MNRAS, 396, 918

Matsuura M. et al., 2011, Science, 333, 1258

Matsuura M. et al., 2015, ApJ, 800, 50

Matthews B., Wallace B., Taylor A., 1998, ApJ, 20, 312

McClure-Griffiths N. M., Green A. J., Dickey J. M., Gaensler B. M., Haynes R. F., Wieringa M. H., 2001, ApJ, 551, 394

Minami S., Ota N., Yamauchi S., Koyama K., 2013, PASJ, 65, 99

Misanovic Z., Kargaltsev O., Pavlov G. G., 2010, ApJ, 725, 931

Moffett D., Gaensler B., Green A., Slane P., Harrus I., Dodson R., 2002, in Slane P. O., Gaensler B. M., eds, ASP Conf. Ser. Vol. 271, Neutron Stars in Supernova Remnants. Astron. Soc. Pac., San Francisco, p. 221

Molinari S. et al., 2010, PASP, 122, 314

Molinari S. et al., 2016, A\&A, 591, A149

Morgan H. L., Edmunds M. G., 2003, MNRAS, 343, 427

Morton T. D., Slane P., Borkowski K. J., Reynolds S. P., Helfand D. J., Gaensler B. M., Hughes J. P., 2007, ApJ, 667, 219

Murakami H. et al., 2007, PASJ, 59, 369

Nehmé C., Kassounian S., Sauvage M., 2019, Exp. Astron., 48, 1

Ng C. Y., Bucciantini N., Gaensler B. M., Camilo F., Chatterjee S., Bouchard A., 2012, ApJ, 746, 1

Nugent J. J., Pravdo S. H., Garmire G. P., Becker R. H., Tuohy I. R., Winkler P. F., 1984, ApJ, 284, 612 
Nynka M. et al., 2013, ApJ, 778, 1

Odegard N., 1986, ApJ, 301, 813

Omand C., Kashiyama K., Murase K., 2019, MNRAS, 484, 5468

Otsuka M. et al., 2010, A\&A, 518, L139

Paladini R. et al., 2012, ApJ, 760, 149

Pannuti T. G., Rho J., Borkowski K. J., Cameron P. B., 2010, AJ, 140, 1787

Pannuti T. G., Rho J., Heinke C. O., Moffitt W. P., 2014a, AJ, 147, 55

Pannuti T. G., Kargaltsev O., Napier J. P., Brehm D., 2014b, ApJ, 782, 102

Park G. et al., 2013, ApJ, 777, 14

Petriella A., Paron S. A., Giacani E. B., 2013, A\&A, 554, A73

Phillips J. P., Marquez-Lugo R. A., 2010, MNRAS, 409, 701

Phillips J. P., Ramos-Larios G., Perez-Grana J. A., 2009, MNRAS, 397, 1215

Pihlström Y. M., Sjouwerman L. O., Frail D. A., Claussen M. J., Mesler R. A., McEwen B. C., 2014, AJ, 147, 73

Pilbratt G. L. et al., 2010, A\&A, 518, L1

Pinheiro Goncalves D. et al., 2011, AJ, 142, 42

Povich M. S. et al., 2007, ApJ, 660, 346

Purcell C. R. et al., 2012, MNRAS, 426, 1972

Pye J. P., Becker R. H., Seward F. D., Thomas N., 1984, MNRAS, 207, 649

Rakowski C. E., Hughes J. P., Slane P., 2001, ApJ, 548, 258

Rakowski C. E., Badenes C., Gaensler B. M., Gelfand J. D., Hughes J. P., Slane P. O., 2006, ApJ, 646, 982

Ranasinghe S., Leahy D. A., 2018a, AJ, 155, 204

Ranasinghe S., Leahy D. A., 2018b, MNRAS, 477, 2243

Reach W. T. et al., 2005, PASP, 117, 978

Reach W. T. et al., 2006, AJ, 131, 1479

Reich W., Furst E., Sofue Y., 1984, A\&A, 133, L4

Reich W., Zhang X., Furst E., 2003, A\&A, 408, 961

Reynolds M. T. et al., 2013, ApJ, 766, 112

Reynolds S. P., 2006, ApJ, 652, L45

Reynoso E. M., Mangum J. G., 2000, ApJ, 20, 874

Reynoso E. M., Johnston S., Green A. J., Koribalski B. S., 2006, MNRAS, 369,416

Rho J., Borkowski K. J., 2002, ApJ, 575, 44

Rho J., Petre R., 1998, ApJ, 503, L167

Rho J., Hewitt J. W., Bieging J., Reach W. T., Andersen M., Güsten R., 2017, ApJ, 834, 12

Rho J. et al., 2018, MNRAS, 479, 5101

Rowlands K., Gomez H. L., Dunne L., Aragón-Salamanca A., Dye S., Maddox S., da Cunha E., van der Werf P., 2014, MNRAS, 441, 1040

Roy S., Pal S., 2013, PIAU, 9, 197

Sabin L. et al., 2013, MNRAS, 431, 279

Saken J. M., Fesen R. A., Shull J. M., 1992, ApJ SS, 81, 715

Sankrit R. et al., 2010, ApJ, 712, 1092

Sarma A., Goss W., Green A., Frail D. A., 1997, ApJ, 483, 335

Sasaki M., Plucinsky P. P., Gaetz T. J., Bocchino F., 2013, A\&A, 552, A45

Sato F., 1979, ApL, 20, 43

Sato T., Koyama K., Lee S. H., Takahashi T., 2016, PASJ, 68, S81

Sezer A., Gök F., 2014, ApJ, 790, 5

Shan S. S., Zhu H., Tian W. W., Zhang M. F., Zhang H. Y., Wu D., Yang A. Y., 2018, ApJS, 238, 35

Shaver P. A., Salter C. J., Patnaik A., van Gorkom J. H., Hunt G. C., 1985, Nature, 313, 113

Simpson R. J. et al., 2012, MNRAS, 424, 2442

Sjouwerman L. O., Pihlström Y. M., Fish V. L., 2010, ApJ, 739, L111

Slane P. et al., 2012, ApJ, 749, 131

Smith A., Jones L. R., Peacock A., Pye J. P., 1985, ApJ, 296, 469

Stupar M., Parker Q. A., 2011, MNRAS, 414, 2282

Surnis M. P., Joshi B. C., Maan Y., Krishnakumar M. A., Manoharan P. K., Naidu A., 2016, ApJ, 826, 184

Takata A., Nobukawa M., Uchida H., Tsuru T. G., Tanaka T., Koyama K., 2016, PASJ, 68, S31

Tawara Y., Yamauchi S., Awaki H., Kii T., Koyama K., Nagase F., 1988, in Tanaka Y., ed., Physics of Neutron Stars and Black Holes, p. 71

Temim T., Sonneborn G., Dwek E., Arendt R. G., Gehrz R. D., Slane P., Roellig T. L., 2012, ApJ, 753, 72
Temim T., Dwek E., Tchernyshyov K., Boyer M. L., Meixner M., Gall C., Roman-Duval J., 2015, ApJ, 799, 158

Temim T., Dwek E., Arendt R. G., Borkowski K. J., Reynolds S. P., Slane P., Gelfand J. D., Raymond J. C., 2017, ApJ, 836, 129

The Astropy Collaboration et al., 2013, A\&A, 558, A33

The Astropy Collaboration et al., 2018, AJ, 156, 123

The L. S. et al., 2006, A\&A, 450, 1037

Tian W. W., Leahy D. A., 2006, A\&A, 455, 1053

Tian W. W., Leahy D. A., 2008, MNRAS, 58, 54

Tian W. W., Leahy D. A., 2012, MNRAS, 421, 2593

Tian W. W., Leahy D. A., 2014, ApJ, 783, L2

Tian W. W., Haverkorn M., Zhang H. Y., 2007a, MNRAS, 378, 1283

Tian W. W., Li Z., Leahy D. A., Wang Q. D., 2007b, ApJ, 657, L25

Tian W. W., Leahy D. A., Li D., 2010, MNRAS, 404, L1

Torii K., Uchida H., Hasuike K., Tsunemi H., Yamaguchi Y., Shibata S., 2006, PASJ, 58, 11

Townsley L. K., Broos P. S., Garmire G. P., Anderson G. E., Feigelson E. D., Naylor T., Povich M. S., 2018, ApJS, 235, 43

Tsuboi M., Miyazaki A., Uehara K., 2015, PASJ, 67, 1091

Tsuji N., Uchiyama Y., 2014, PASJ , 68, 108

Uyaniker B., Kothes R., Brunt C. M., 2002, ApJ, 565, 1022

van den Bergh S., 1991, Phys. Rep., 204, 385

van den Bergh S., 1993, Comments Astrophys., 17, 125

Vink J., 2004, ApJ, 604, 7

Voisin F., Rowell G., Burton M. G., Walsh A., Fukui Y., Aharonian F., 2016, MNRAS, 458, 2813

Werner M. W. et al., 2004, ApJS, 154, 1

Whiteoak J. B. Z., 1992, MNRAS, 256, 121

Whiteoak J. B. Z., Green A. J., 1996, A\&AS, 118, 329

Williams B. J. et al., 2011, ApJ, 729, 65

Willingale R., Bleeker J. A. M., van der Heyden K. J., Kaastra J. S., 2003, A\&A, 398, 1021

Wolszczan A., Cordes J. M., Dewey R. J., 1991, ApJ, 372, L99

Wootten A., 1981, ApJ, 245, 105

Xu J.-L., Wang J.-J., 2012, A\&A, 543, A24

Yamaguchi H., Ueno M., Koyama K., Bamba A., Yamauchi S., 2004, PASJ, 56,1059

Yamaguchi H., Tanaka M., Maeda K., Slane P. O., Foster A., Smith R. K., Katsuda S., Yoshii R., 2012, ApJ, 749, 137

Yamaguchi H. et al., 2015, ApJ, 801, L31

Yamauchi S., Minami S., Ota N., Koyama K., 2014, ASJ, 66, 1

Yar-Uyaniker A., Uyaniker B., Kothes R., 2004, ApJ, 616, 247

Yasumi M., Nobukawa M., Nakashima S., Uchida H., Sugawara R., Tsuru T. G., Tanaka T., Koyama K., 2014, PASJ, 66, 1

Yusef-Zadeh F., Goss W., 1999, ApJ, 527, 172

Yusef-Zadeh F., Morris M., 1987, ApJ, 320, 545

Yusef-Zadeh F., Melia F., Wardle M., 2000, Science, 287, 85

Zajczyk A. et al., 2012, A\&A, 542, A12

Zhang G.-Y., Chen Y., Su Y., Zhou X., Pannuti T. G., Zhou P., 2015, ApJ, 799,103

Zhao J. H., Morris M. R., Goss W. M., 2013, ApJ, 777, 1

Zhou P., Chen Y., 2011, ApJ, 743, 4

Zhou P., Safi-Harb S., Chen Y., Zhang X., Jiang B., Ferrand G., 2014, ApJ, 791, 87

Zhou P., Chen Y., Safi-Harb S., Zhou X., Sun M., Zhang Z.-Y., Zhang G.-Y., 2016, ApJ, 831, 192

Zhu H., Tian W. W., Torres D. F., Pedaletti G., Su H. Q., 2013, ApJ, 775, 95

Zhu H., Tian W. W., Zuo P., 2014, ApJ, 793, 95

Zoglauer A. et al., 2015, ApJ, 798, 4

\section{APPENDIX A: FIR CATALOGUE OF SNRS WITH HERSCHEL}



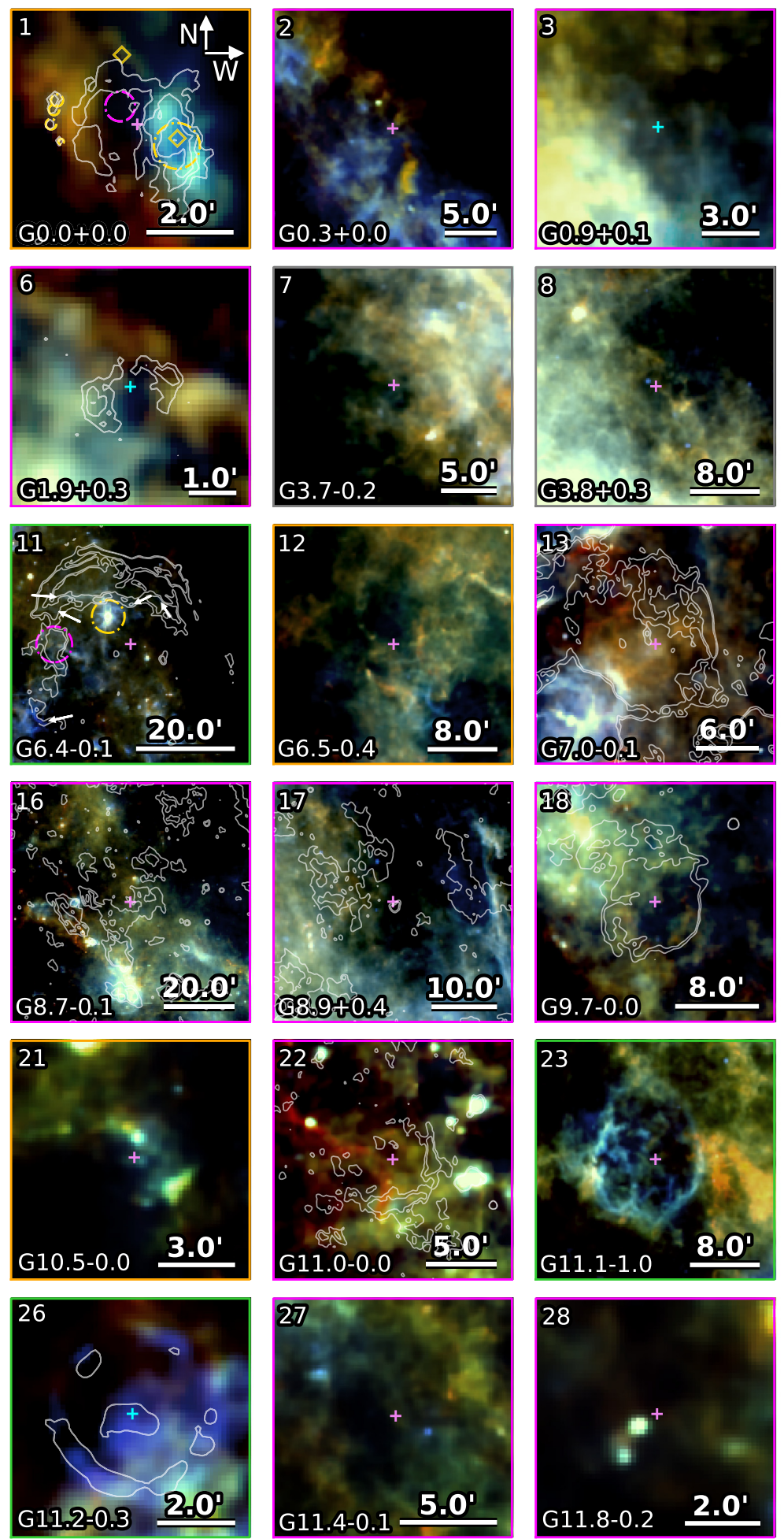
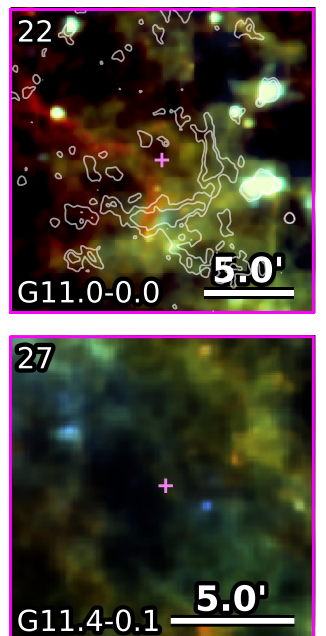
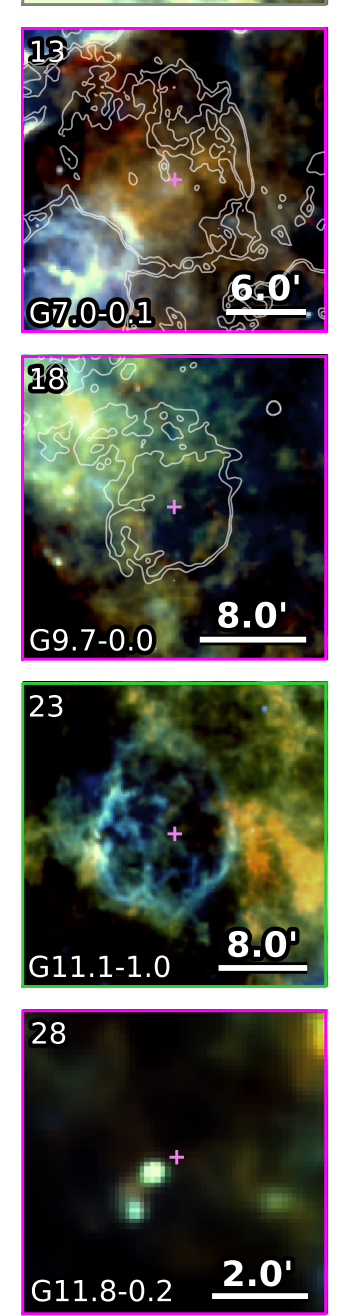
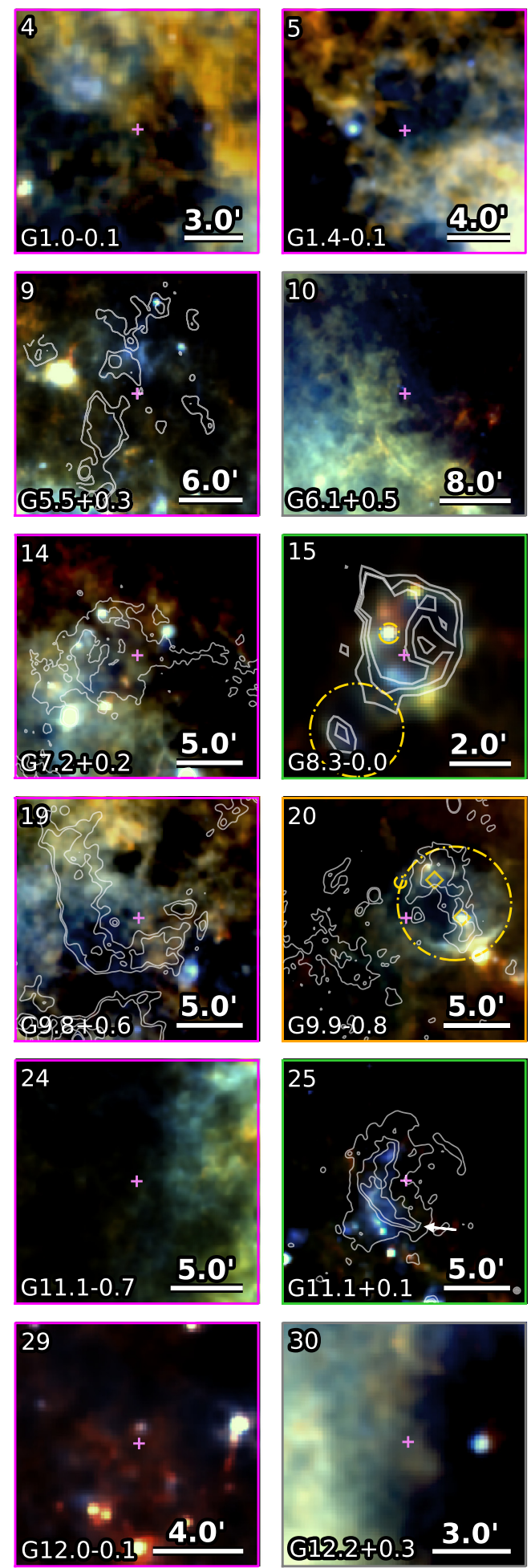
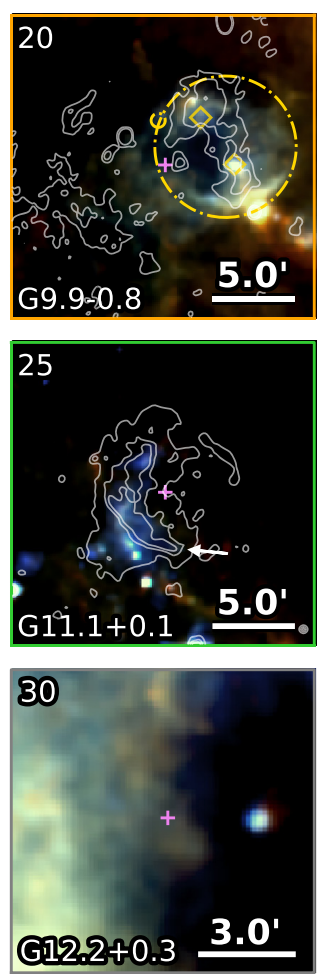

Figure A1. Herschel three colour images of the locations of all supernova remnants in our sample. Colours are red $=250 \mu \mathrm{m}$, green $=160 \mu \mathrm{m}$, and blue $=70 \mu \mathrm{m}$. Purple crosses indicate the source radio centre from Green (2014) and cyan crosses indicate the X-ray centre. The border colour indicates the associated detection level in this study, as given in Table A1, where green = level 1, orange = level 2, magenta = level 3, grey = level 4, and black $=$ unstudied In some panels dashed magenta circles and white arrows indicate particular FIR dust emission features associated with the SNR, gold circles indicate unrelated structure such as $\mathrm{H}$ II regions. Overlaid on to G0.0+0.0, the gold diamonds indicate the location of the associated neutron star and Sgr A*. The gold diamonds on the image of G9.9-0.8 indicate the locations of H II regions. VLA GPS $20 \mathrm{~cm}$ contours overlaid on to the images of G0.0+0.0, G7.0-0.1, G7.2+0.2, G8.3-0.0, G9.7-0.0, G9.8+0.6, and G11.0-0.0; Chandra contours are overlaid on to G1.9+0.3 and G11.2-0.3; 90 cm contours are overlaid on to G5.5+0.3, G6.4-0.1, G8.7-0.1, G8.9+0.4, and G9.9-0.8. 

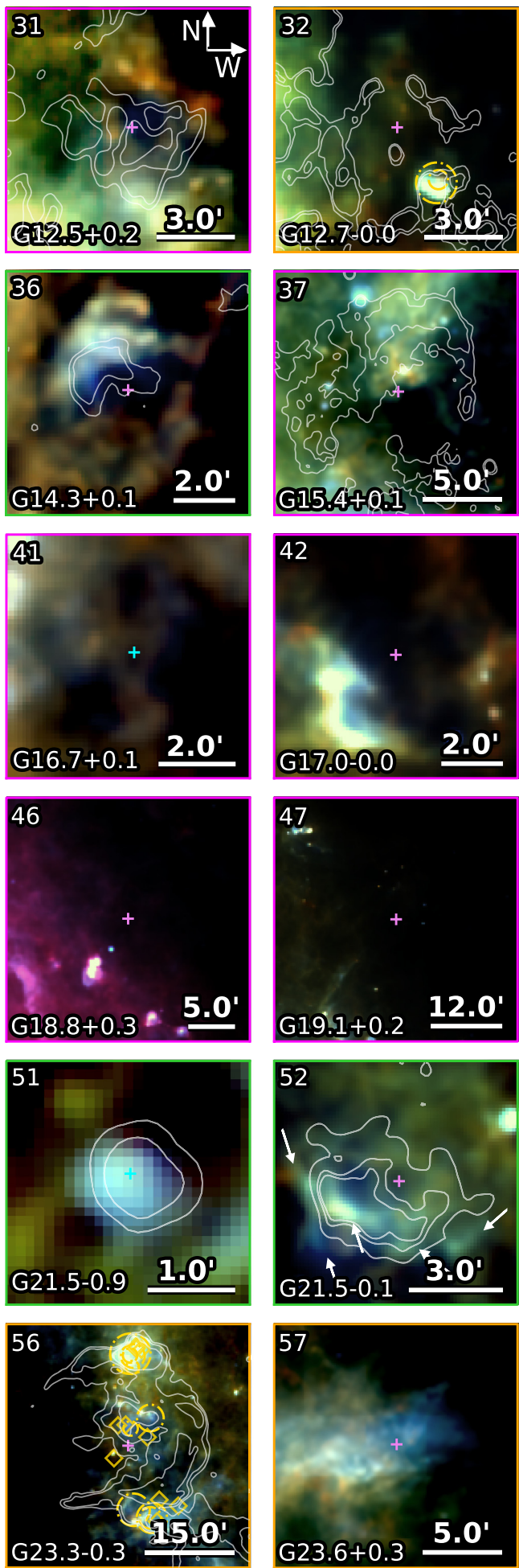
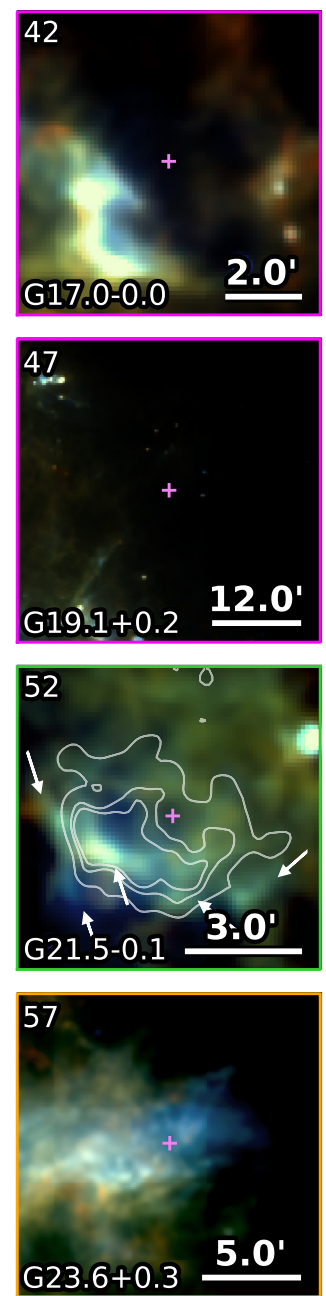
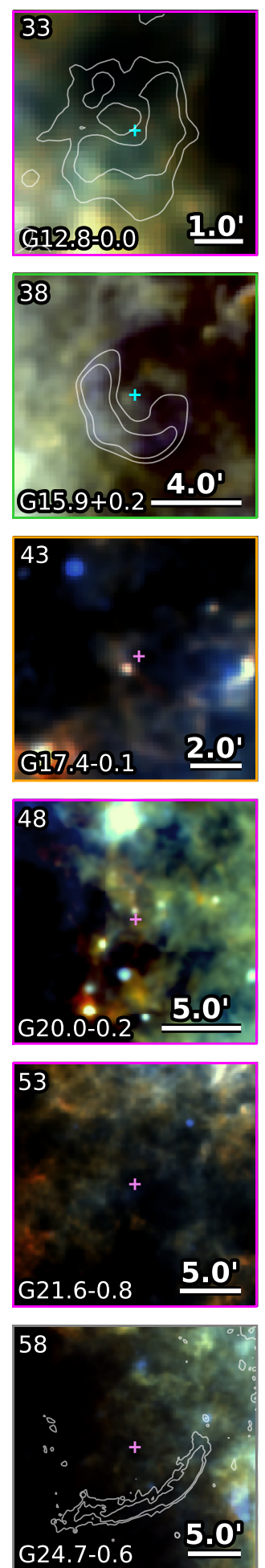
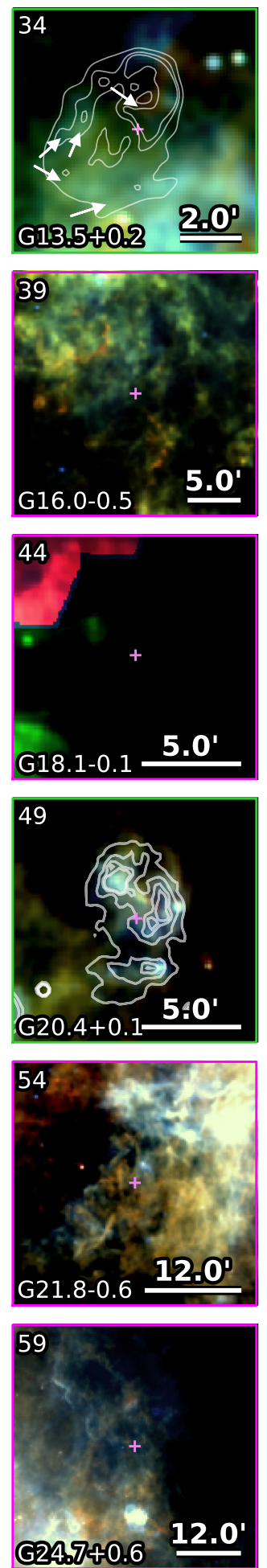
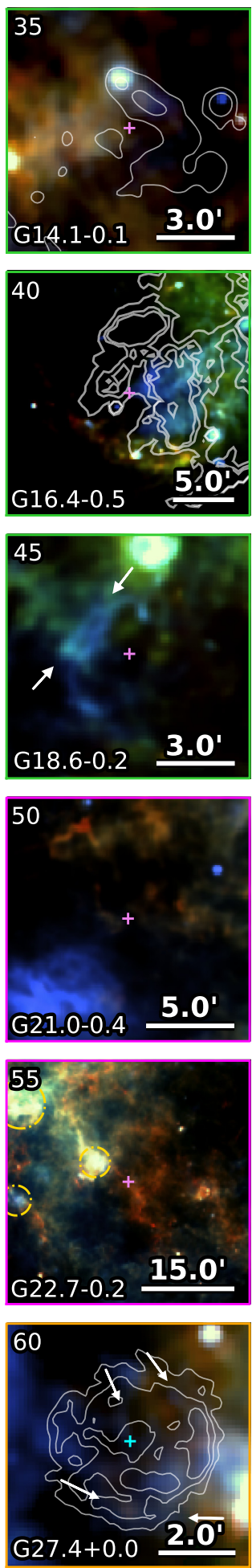

Figure A1 - continued. VLA GPS 20 cm contours are overlaid on to G12.5+0.2, G12.7-0.0, G12.8-0.0, G13.5+0.2, G14.3+0.1, G20.4+0.1, and G24.7-0.6; VLA GPS $90 \mathrm{~cm}$ contours are overlaid on to G15.4+0.1; Chandra contours are overlaid on to G15.9+0.2, G21.5-0.9, and G27.4+0.0; MIPS 24 $\mu$ m contours are overlaid on to G14.1-0.1; VGPS $21 \mathrm{~cm}$ contours are overlaid on to G23.3-0.3. The gold diamonds overlaid on to the image of G23.2-0.3 indicate the locations of a gamma-ray source (HESS J1834-087), OH (1720 MHz) maser lines, and other maser emission. 

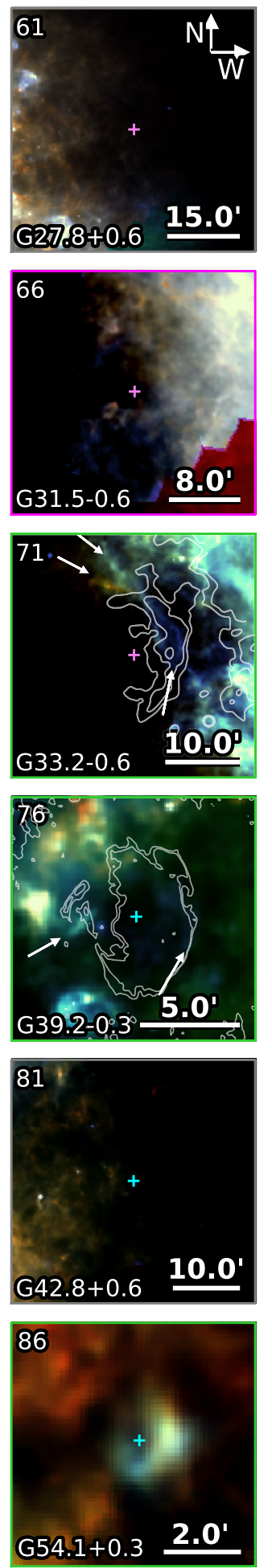
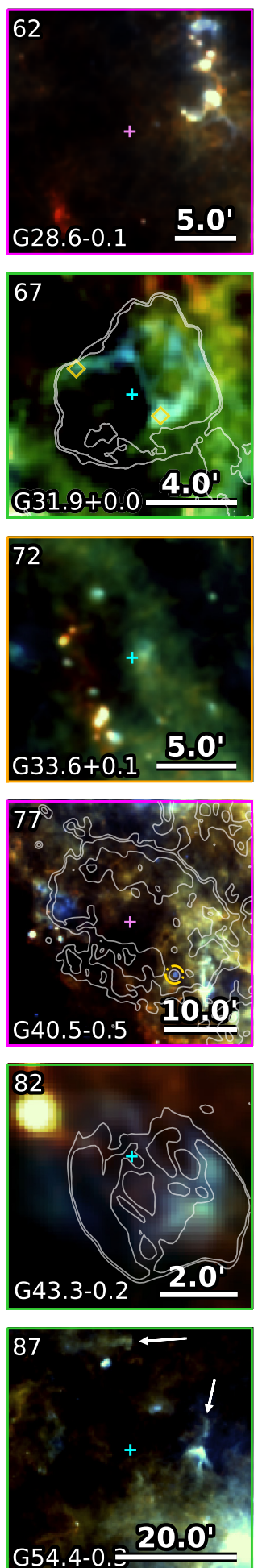
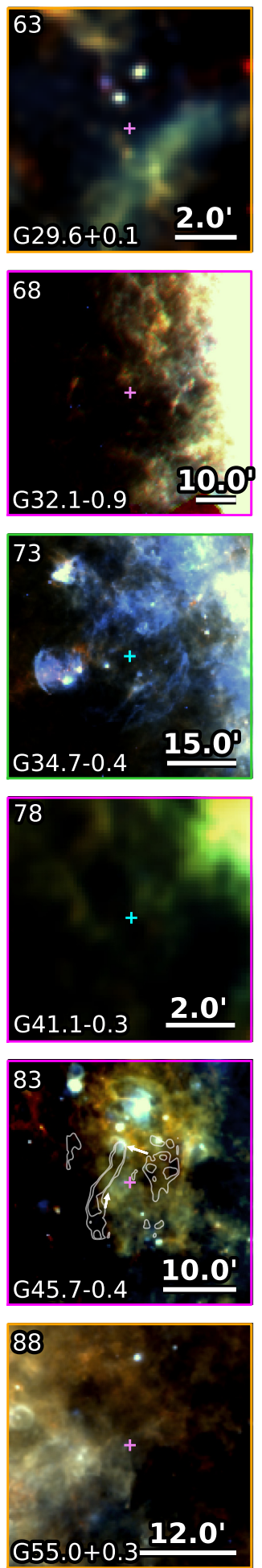
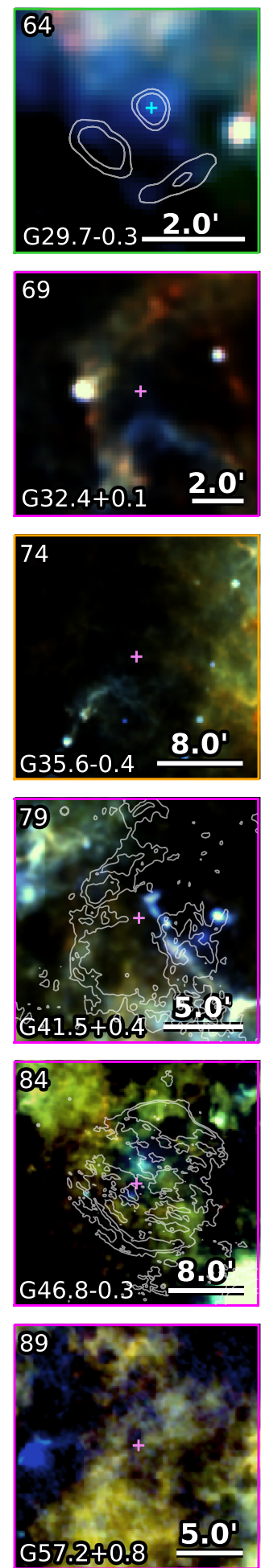
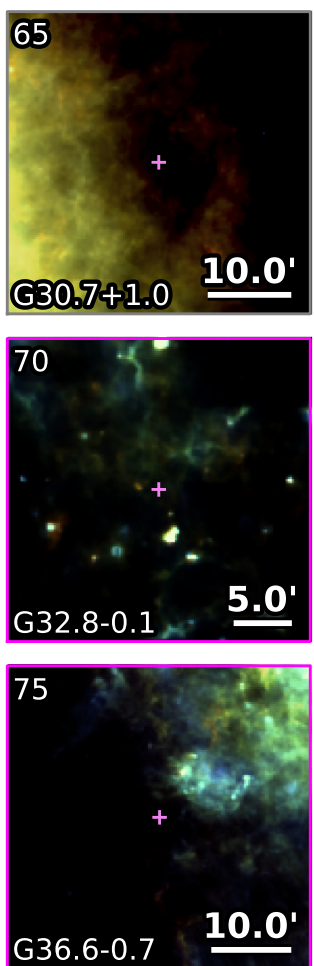

80
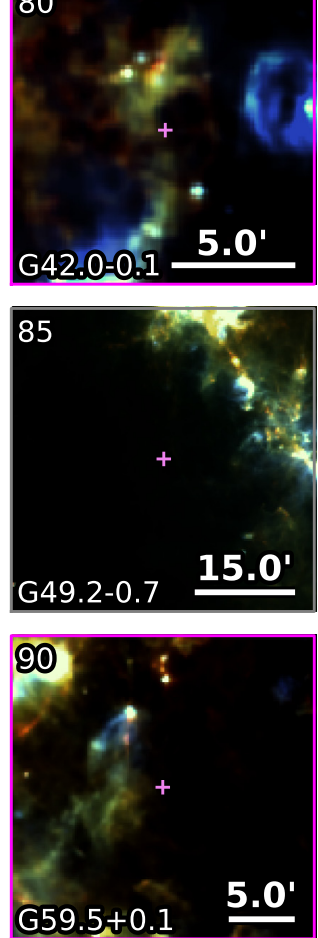

Figure A1 - continued. Chandra contours are overlaid on to G29.7-0.3 and G33.6+0.1; VLA GPS $20 \mathrm{~cm}$ contours are overlaid on to G31.9+0.0, G33.2-0.6, G39.2-0.3, G41.5+0.4 G43.3-0.2, and G46.8-0.7; VGPS $21 \mathrm{~cm}$ contours are overlaid on to G40.5-0.5; NVSS 1.4 GHz contours are overlaid on to G45.7-0.4. 

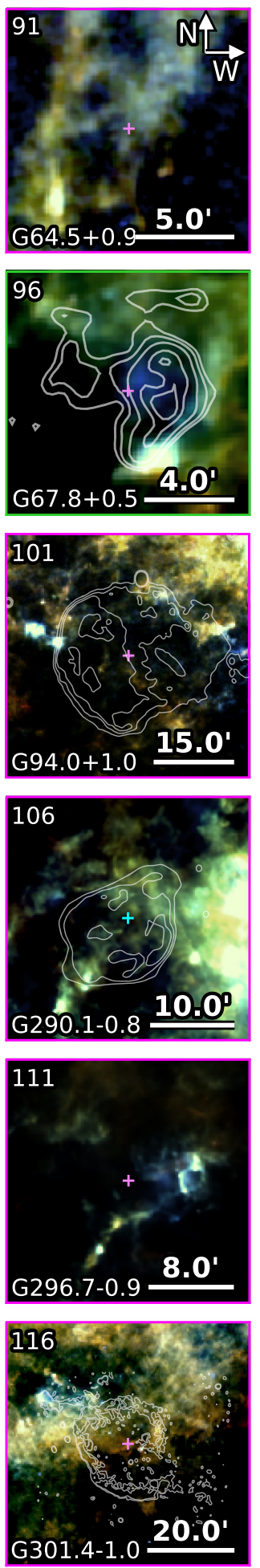
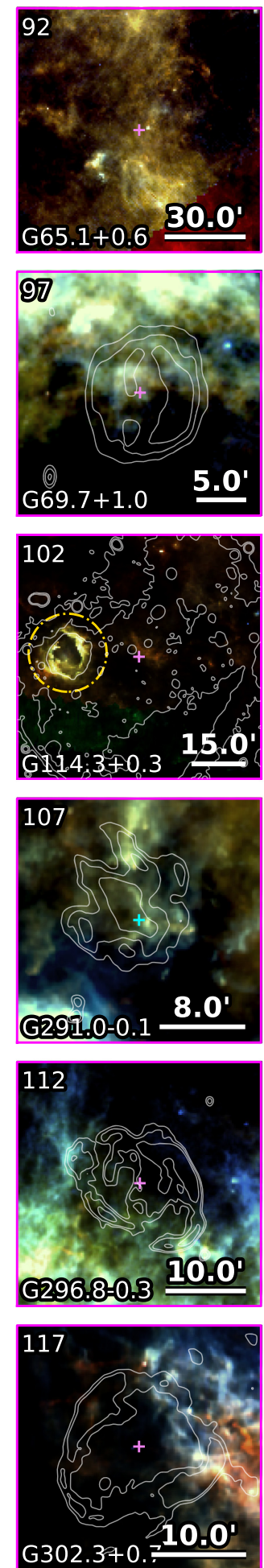
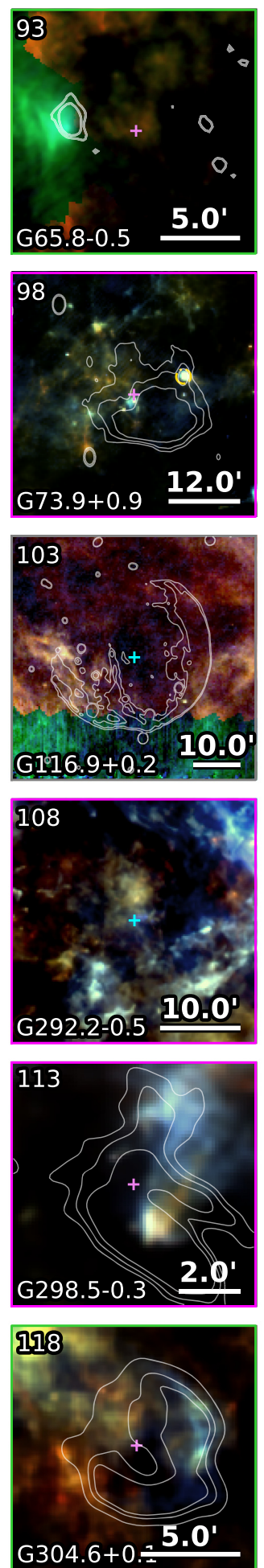
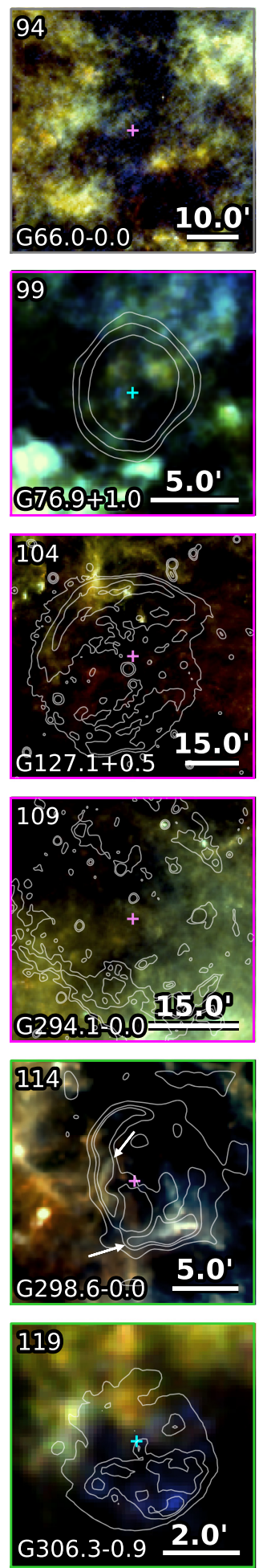
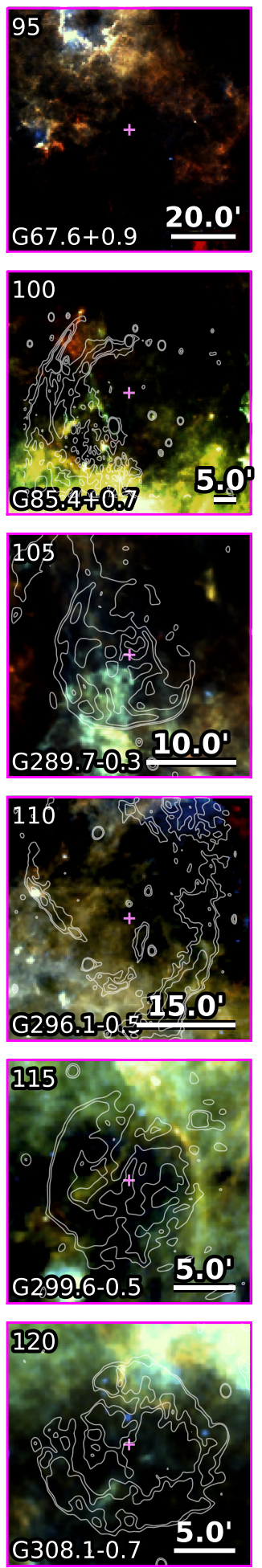

Figure A1 - continued. NVSS 1.4 GHz contours are overlaid on to G65.8-0.5, and G67.8+0.5. Canadian Galactic Plane Survey (CGPS, 1420 MHz) contours are overlaid on to G69.7+1.0, G73.9+0.9, G76.9+1.0, G85.4+0.7, G94.0+1.0, G114.3+0.3, G116.9+0.2, and G127.1+0.5. MOST 843 MHz contours are overlaid on to G289.7-0.3, G290.1-0.8, G291.0-0.1, G294.1-0.0, G296.1-0.5, G296.8-0.3, G298.5-0.3, G298.6-0.0, G299.6-0.5, G301.4-1.0, G302.3+0.7, G304.6+0.1, and G308.1-0.7; Chandra contours are overlaid on to G306.3-0.9. 

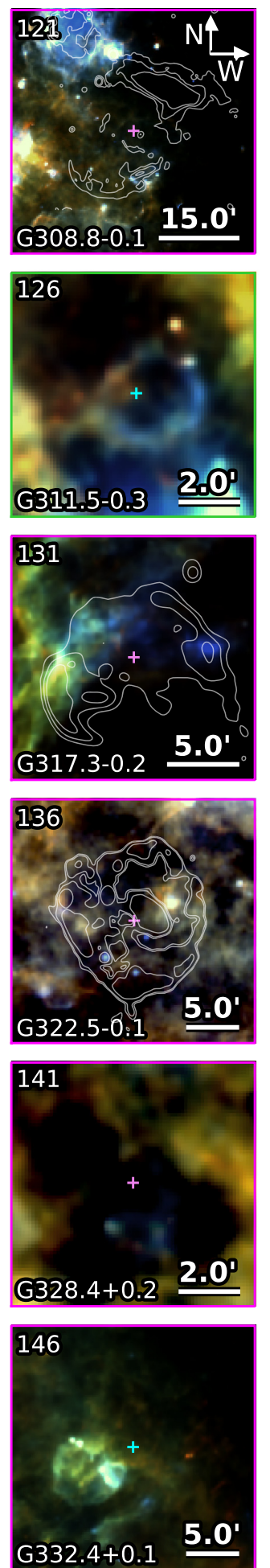
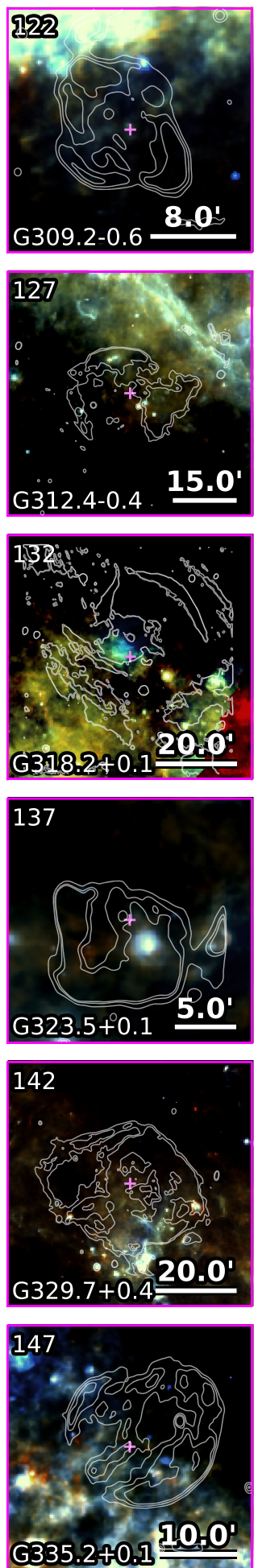
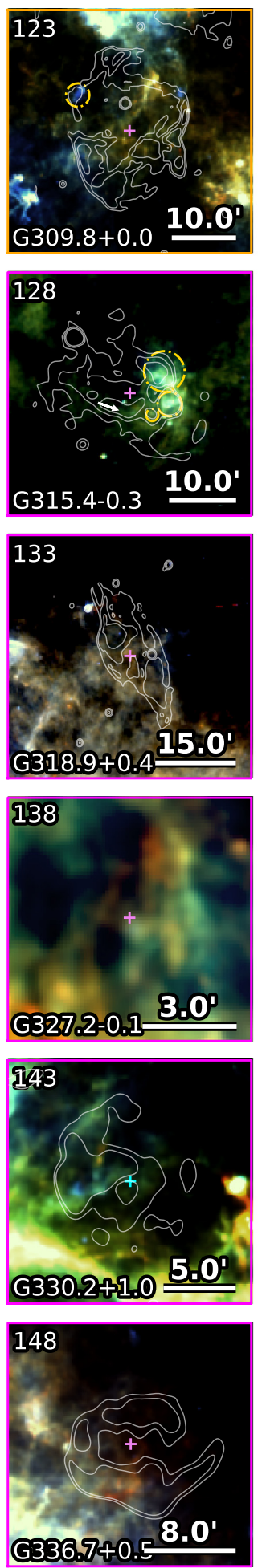
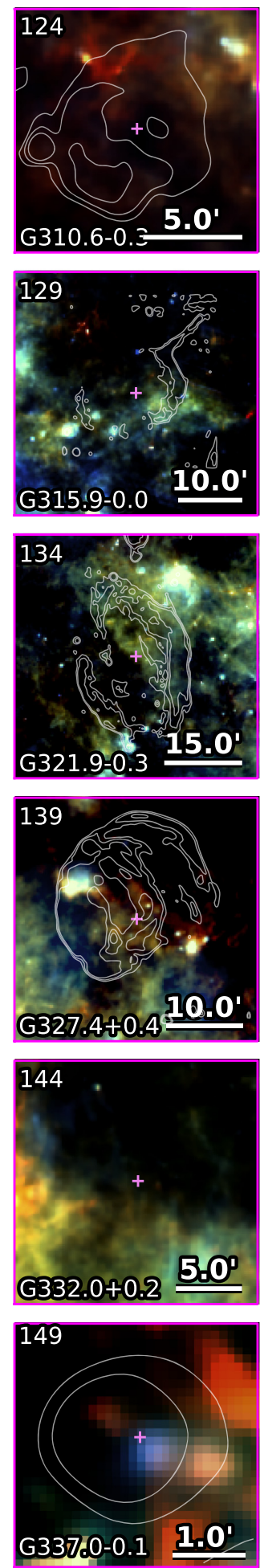
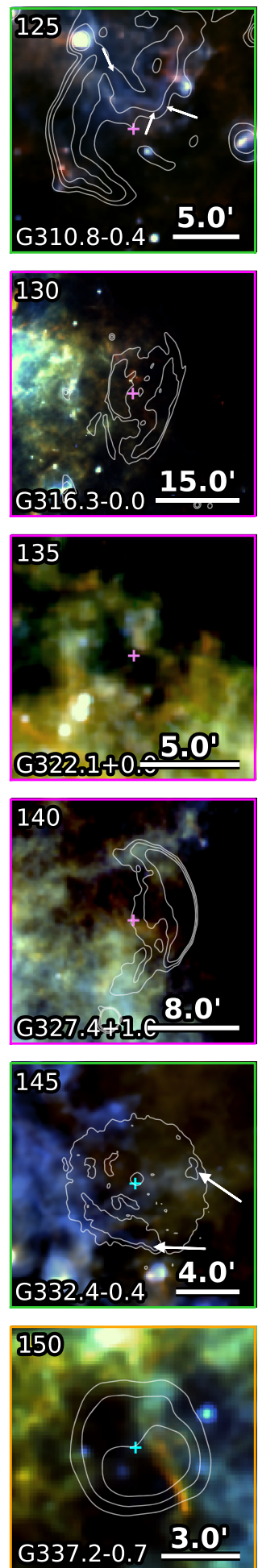

Figure A1 - continued. Chandra contours are overlaid on to G332.4-0.4, all other contours are MOST 843 MHz. 

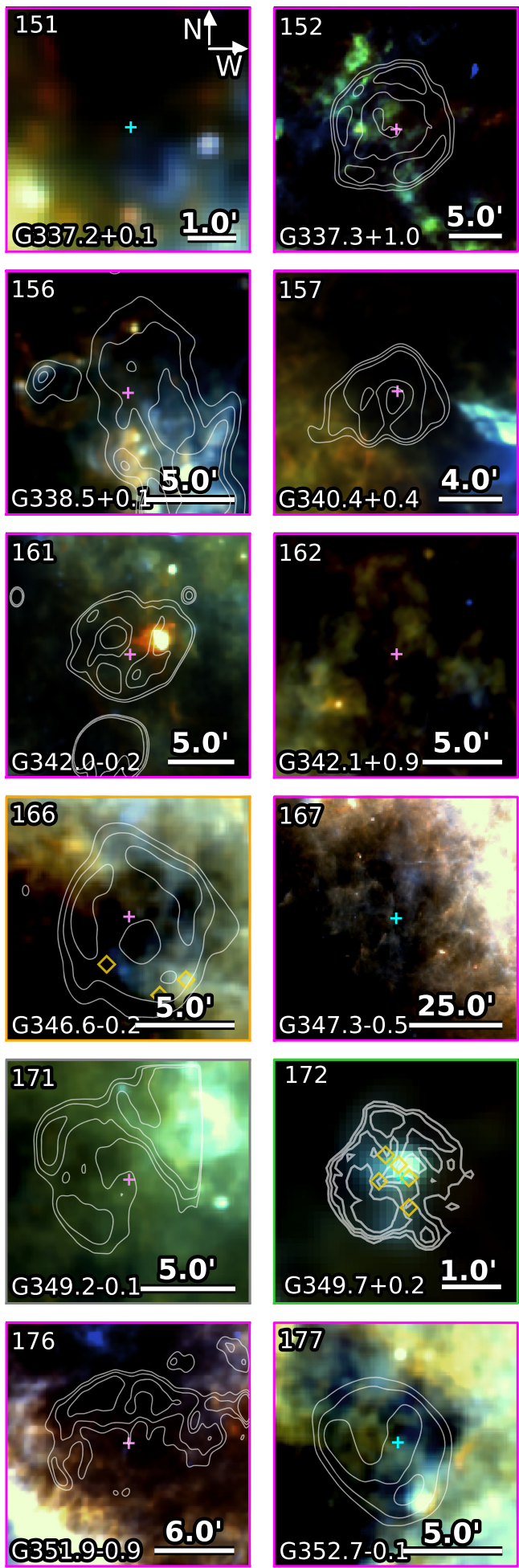
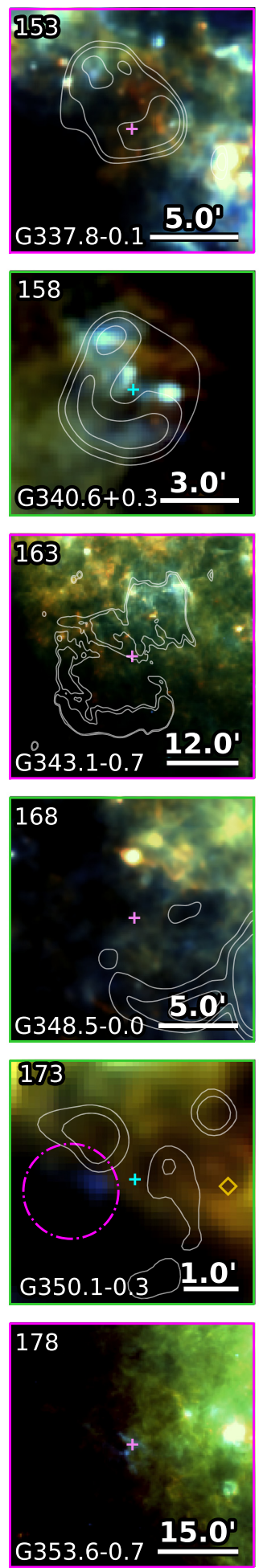
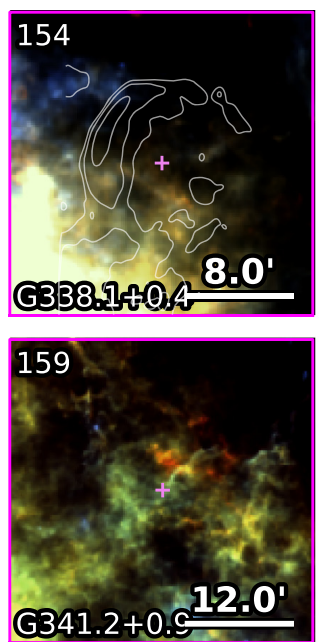
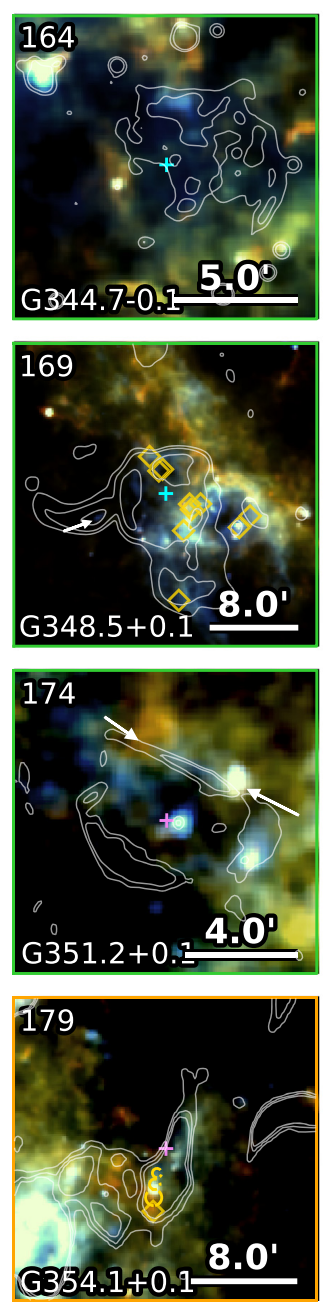
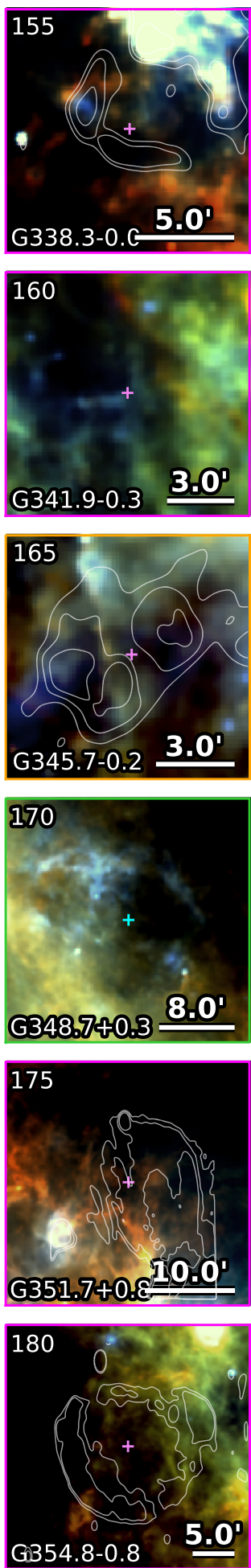

Figure A1 - continued. MOST 843 MHz contours are overlaid on to G337.2-0.7, G342.2-0.2, G343.1-0.7, G345.7-0.2, G346.6-0.2, G348.5-0.0, G348.5+0.1, G349.2-0.1, G351.2+0.1, G351.7+0.8, G351.9-0.9, G352.7-0.1, G3541+0.1, and G354.8-0.8; MIPS 24 um contours are overlaid on to G344.7-0.1 and G350.1-0.3; Chandra contours are overlaid on to G349.7+0.2. The diamonds overlaid on to the images of G350.1-0.3 and G354.1+0.1 indicate the locations of an associated neutron star and pulsar, respectively. All other diamonds indicate the locations of maser emission. 

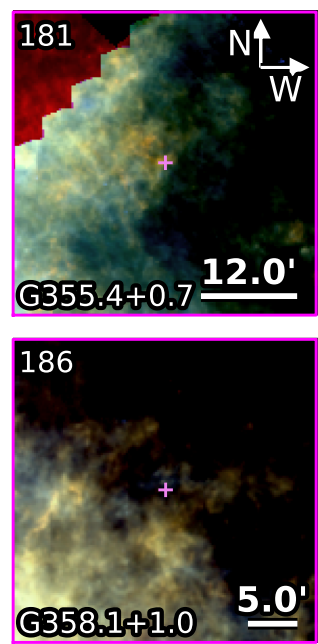
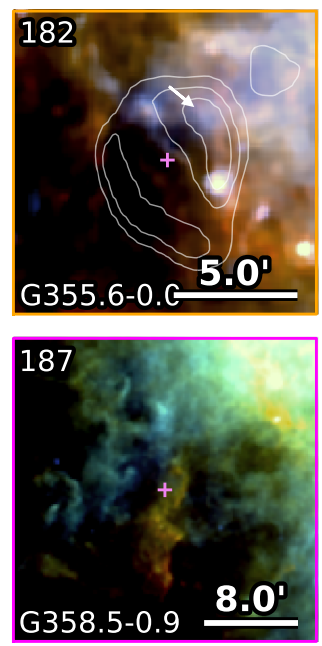
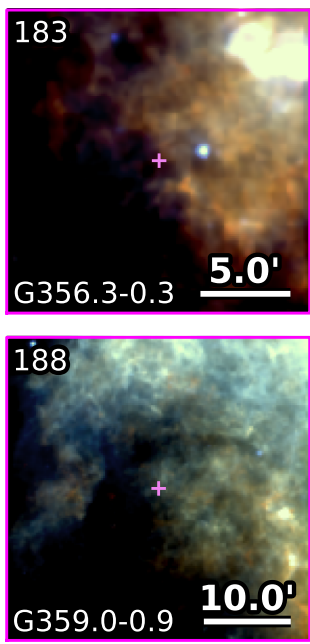
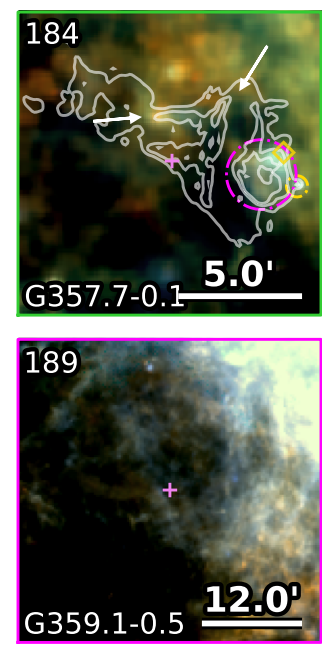

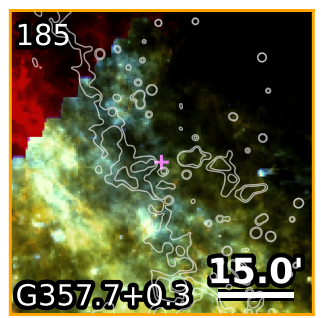

190

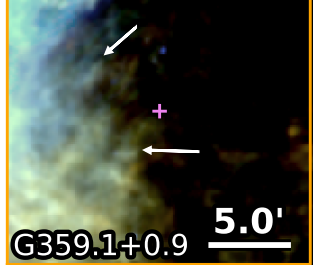

Figure A1 - continued. MOST $843 \mathrm{MHz}$ contours are overlaid on to G355.6-0.0; NVSS 1.4 GHz (white) and Chandra X-ray (cyan) contours are overlaid on to G357.7-0.1; MIPS $24 \mu \mathrm{m}$ contours are overlaid on to G357.7+0.3. The gold diamond overlaid on to the images of G357.7-0.1 and G357.7+0.3 indicate the locations of an $\mathrm{OH}(1720 \mathrm{MHz})$ maser and a Mira star, respectively. 


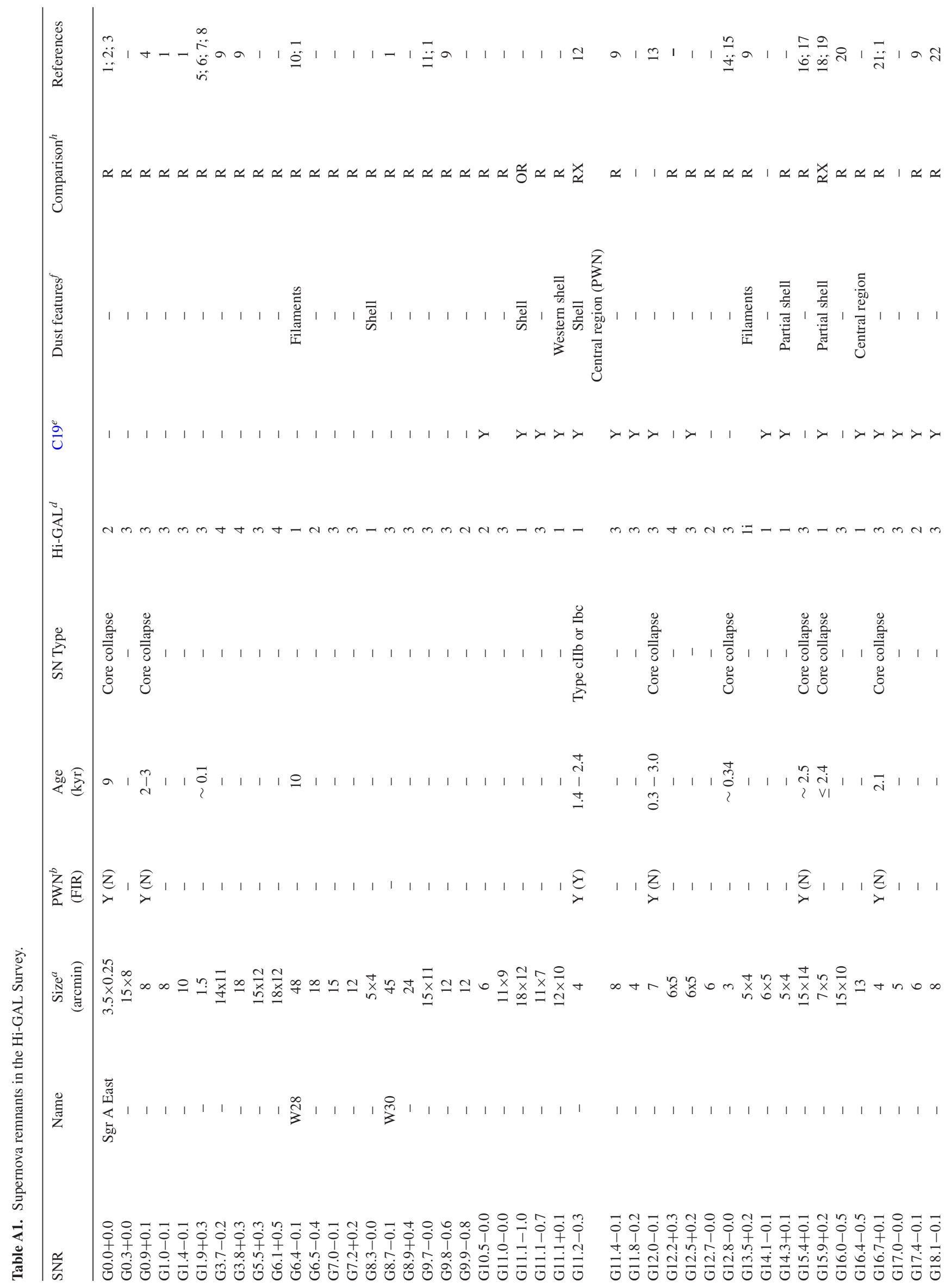




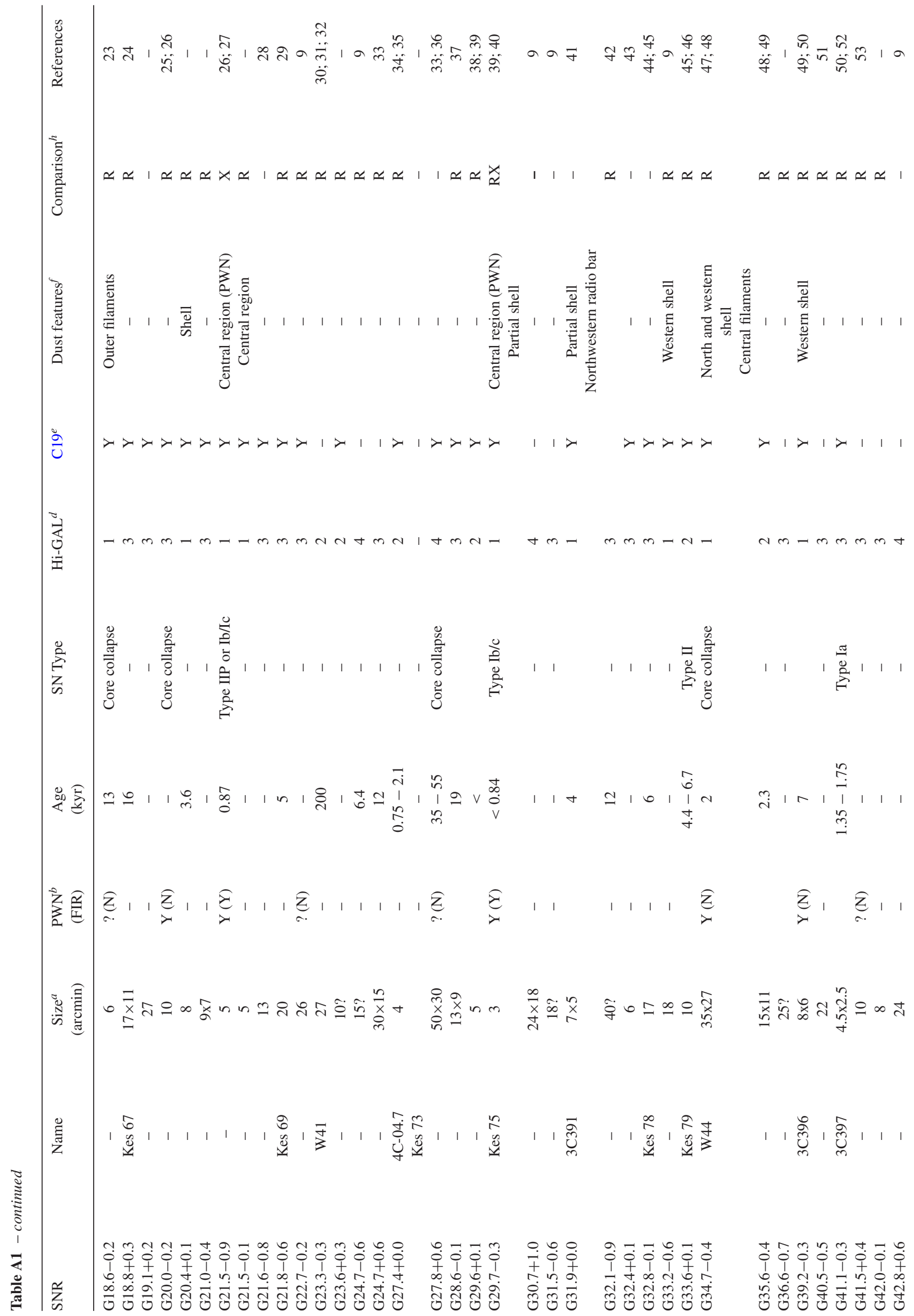




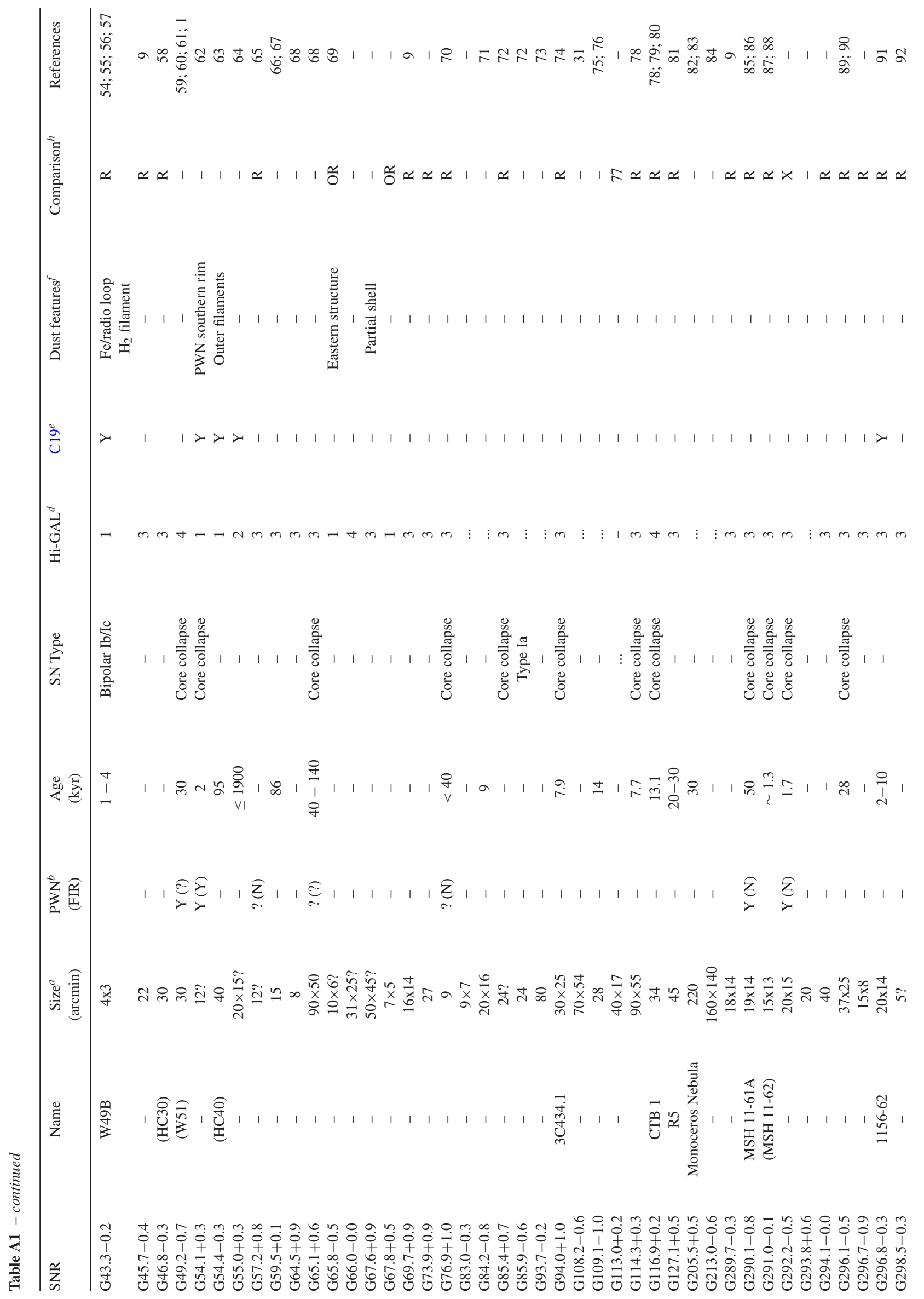




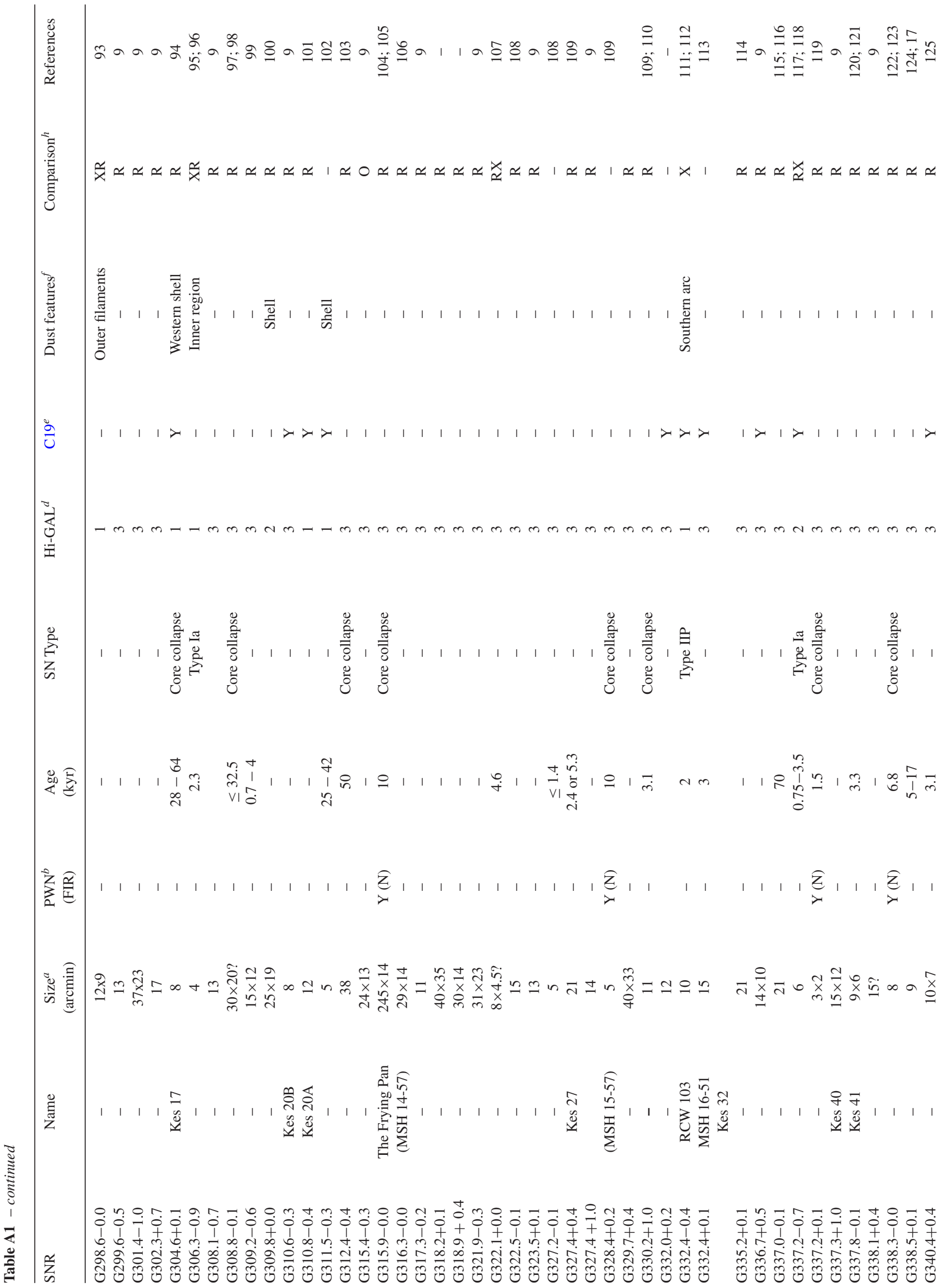




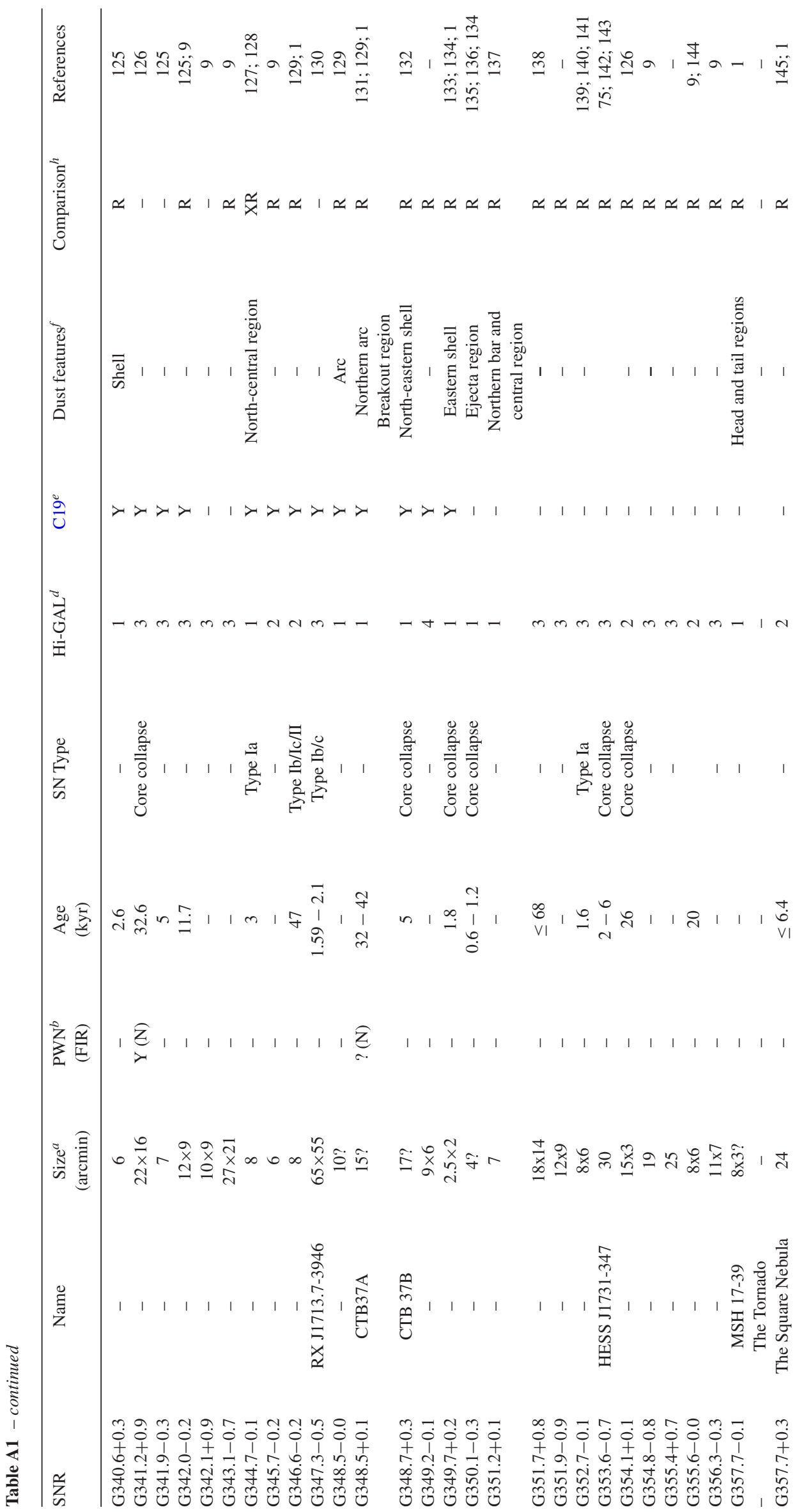




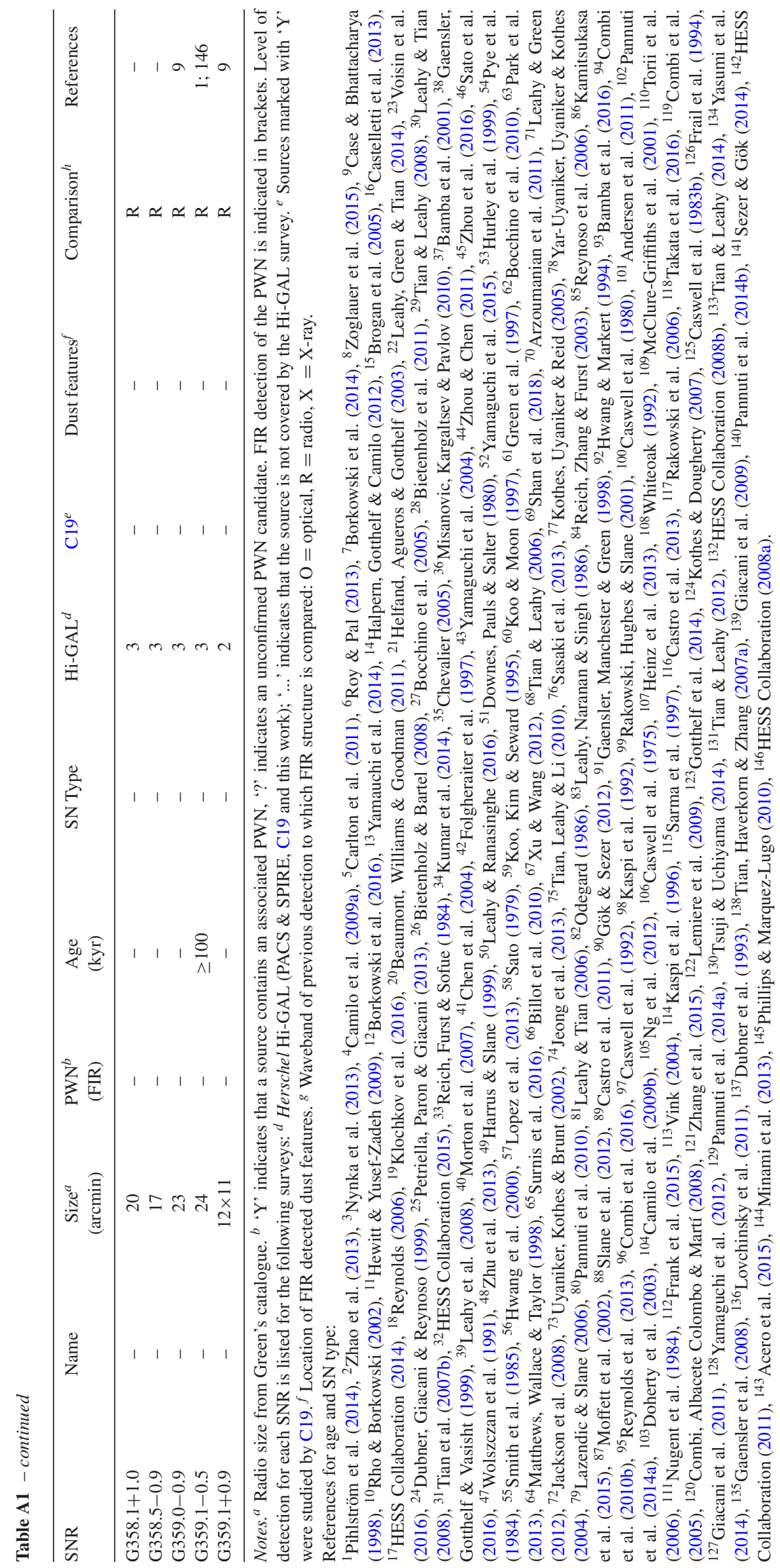




\section{APPENDIX B: DUST TEMPERATURES IN THE LEVEL 1 DETECTED SUPERNOVA REMNANTS}

\section{B1 SNR Temperature map simulations}

Due to the location of the Galactic Plane SNRs, there is significant contamination from foreground/background interstellar emission for most of our sample and many of the sources (even the level 1 detections) are faint in comparison to the surrounding or foreground/background ISM. As such, any dust temperatures and dust masses derived could be significantly affected by this issue, more so than in studies of SNRs in the Large Magellanic Cloud which is a face-on galaxy. This is the biggest limitation when attempting to measure dust masses and temperatures in a statistical sample of Galactic SNe.

We test the effect of this by simulating a dusty SNR for a bright and faint case. Both simulated SNRs with varying temperatures have the same $70 / 160$ colours, but the former is chosen to be high signal-to-noise, well above the brightness of the ISM (the simulated SNR has pixel fluxes up to $\sim 9000$ and $\sim 3200 \mathrm{MJy} \mathrm{sr}^{-1}$ at 70 and $160 \mu \mathrm{m}$, respectively, compared with an average ISM value of $\sim 390$ and $\sim 1370 \mathrm{MJy} \mathrm{sr}^{-1}$ ), and the latter is at a similar level to the brightness of IS clouds seen at off-SNR points in our Hi-GAL maps (with $70 \mu \mathrm{m}$ fluxes in the SNR $\sim 10$ times fainter than the bright case). Our simulation consists of a SNR with an outer shell of dust with temperature $65 \mathrm{~K}$ (to simulate dust heated in forward shock regions) and two cooler components of ejecta dust (at $25 \mathrm{~K}$ and $37 \mathrm{~K}$ ) to simulate cooler ejecta dust similar to the observed PWNe remnants (Chawner et al. 2019) and a Cassiopeia A-like SNR (Barlow et al. 2010; De Looze et al. 2017).

Using equation (1) on the simulated SNR recovers the input temperatures perfectly. The simulated source is then placed on to a region of the Hi-GAL survey at $\alpha=18^{\mathrm{h}} 07^{\mathrm{m}} 58^{\mathrm{s}}, \delta=-19^{\circ} 27^{\prime} 16^{\prime \prime}$ $(l=10.7537, b=0.364)$ as shown in the top panels of Fig. B1, this region is chosen as there is a large variation in the background level, as is the case for several of our level 1 detected SNRs. Equation (1) is then used to create the dust temperature map. The output dust temperatures are significantly biased towards lower temperatures than the input simulation. Across the map, the dust temperatures are lower by $5-15 \mathrm{~K}$ compared to the simulated source, simply as a result of placing the simulated SNR on to a region with background interstellar emission. This situation is further compounded if we take the fainter simulated SNR (which is much more comparable to our real detection level 1 sources). Here the output temperatures range from 20 to $30 \mathrm{~K}$ compared to the input $20-65 \mathrm{~K}$, with an offset of $30 \mathrm{~K}$ in the shell. For the faint source simulation, we also see more variation in the output shell temperatures compared to the bright source. It is clear that for fainter dusty SNRs, the ISM contamination affects the output dust temperatures by significant amounts (and ultimately could lead to orders of magnitude errors in any estimate of the dust mass, depending on the dust size and composition).

To address this we test the affects of attempting to remove the background level and re-derive the temperatures using equation (1) on the background subtracted $70-160 \mu \mathrm{m}$ simulated images. The background level for the simulated SNR is estimated, and then subtracted, using the Nebulizer routine ${ }^{8}$ from the CASUTools suite. Nebulizer takes a region around the SNR to model large-scale

${ }^{8} \mathrm{http} / / /$ casu.ast.cam.ac.uk/surveys-projects/software-release/backgroundfiltering
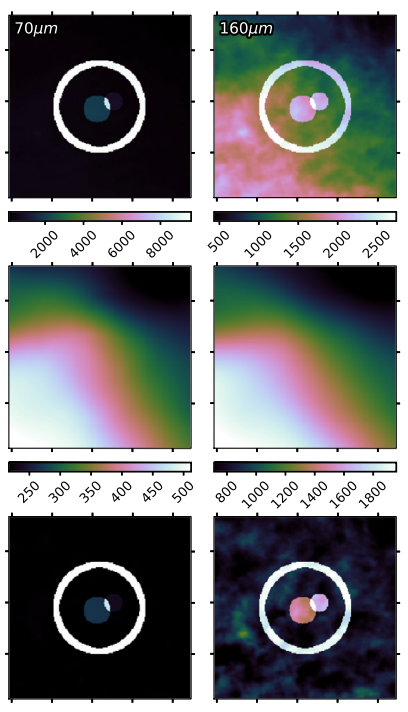

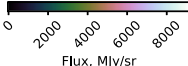
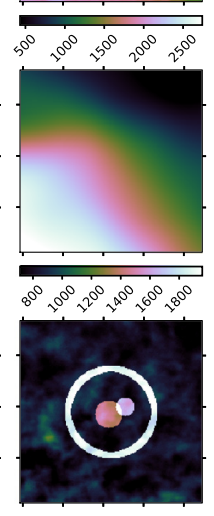

$2^{0} \times 0^{\circ} 0^{\circ} 8^{\circ} \gamma^{\circ} 0^{\circ} \gamma^{\circ}$ Flux, Mylys
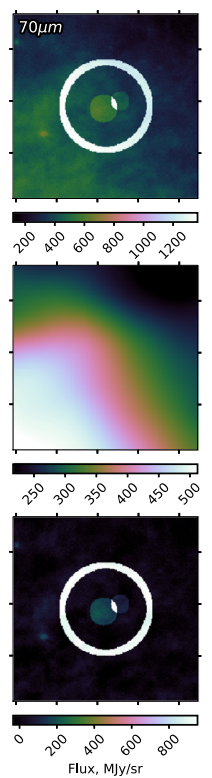

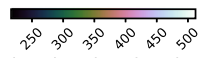

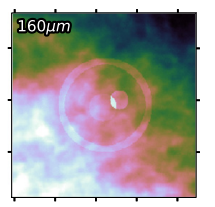

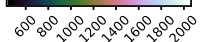
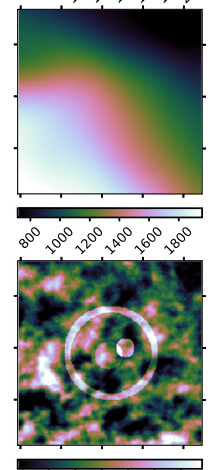

$2,80080.8050$

Flux, Myy/sr
Figure B1. Background subtraction using the Nebulizer routine applied to the bright simulated source (left-hand panel) and the less bright case (right-hand panel). Top: images prior to background subtraction, middle: background estimated by Nebulizer, and bottom: background subtracted image.

variations in the ISM level and then subtract this to return a map with a zero average. We use Nebulizer as it allows us to model variations in the ISM level, rather than assuming a constant level which does not accurately represent the entire region. Nebulizer is applied to the 70 and $160 \mu \mathrm{m}$ images for our simulated SNR, producing the image shown in Fig. B1, and equation (1) is then used on the Nebulizer subtracted 70 and $160 \mu \mathrm{m}$ maps.

For our bright SNR we find that the output temperature map after background subtraction accurately recovers the majority of the input temperatures across the full range of the simulated data (see Fig. B2). An improvement is also seen for the fainter simulated SNR as we are able to identify both warm and cool dust where before we were limited to a very narrow range of cool temperatures. The resulting temperature distribution after background subtraction is wider compared with the input temperatures, as the contamination of the ISM dust in the pixels at a similar brightness level to the simulated SNR has led to a smearing out of the dust temperatures. We also see that for the faint case, the output temperature map is more sensitive to Nebulizer's modelling of large-scale variations in the ISM, there are more small scale features in the output map than in the original simulation.

\section{B2 Notes on the temperature maps for individual remnants}

In this section we study the SNR temperature maps in Fig. 21 which are produced using the 70-160 $\mu \mathrm{m}$ flux ratio after background subtraction, as discussed above. We compare with multiwaveband images to identify the source of elevated dust temperatures, which can be an indicator of heating sources such as ISM-SNR interactions. Because of arbitrary colour scaling in Section 2, some warm dust features may not be evident in the Herschel false colour images, but may be revealed here.

G8.3-0.0: Broad molecular emission $\left({ }^{12} \mathrm{CO}\right)$, flattened radio contours, a possible $\mathrm{OH}$ maser, and a gamma-ray source all suggest interaction with molecular cloud to the north-west (Higashi et al. 

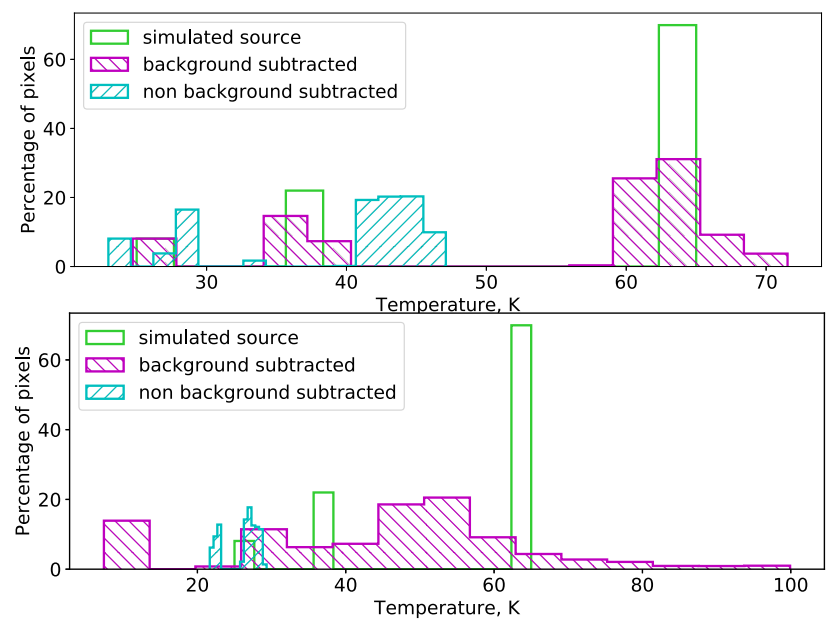

Figure B2. Temperatures within the simulated source in the case of a bright simulated source (top) and a less bright source (bottom). In both cases we find a distribution which is much more similar to the input when we apply a background subtraction.

2008; Kilpatrick et al. 2016). We detect warm dust between $\sim 27$ and $33 \mathrm{~K}$ at the same location of the broad molecular lines observed by Kilpatrick et al. (2016) (their fig. 5) where it is expected that there is a SNR-MC interaction, suggesting that this is heating the dust in this region.

G11.1+0.1: We find evidence of warm dust $(\sim 24-30 \mathrm{~K})$ in the region of this SNR and dust within the magenta circle in Fig. 21 to the north is found within the radio contours. The warmest material, towards the south, does not correlate with the radio structure.

G16.4-0.5: There is warm dust $(\sim 23-28 \mathrm{~K})$, the warmest of which is offset to the north-east of the $70 \mu \mathrm{m}$ bright region, indicated by the magenta circle in Fig. 21. The warm dust is in a similar location to radio structure although its morphology differs making it difficult to determine if the origin of the two is the same.

We do not see evidence that the Herschel shell around the south and western extent of the SNR has any variation in temperature compared with unrelated dust in the region.

G20.4+0.1: We find some warm dust $(\sim 25-27 \mathrm{~K})$ that correlates with the radio structure, although the majority of the warm material has a different structure or is found outside of the radio contours. Anderson et al. (2011) suggested that this source may be a H II region (G20..165, Lockman 1989).

G21.5-0.1: There is evidence of warm dust $(\sim 23-30 \mathrm{~K})$ in this SNR although it does not seem to correlate with the radio structure, as shown in Fig. 21. The warmest region within the radio contours is found in the central region, in a gap in the $160 \mu \mathrm{m}$ structure. There is also warm dust at the edge of the south-eastern radio contours which may be swept up or shock-heated material if it is associated with the SNR.

W49B, G43.3-0.2: Numerous studies of CO, HI, shocked $\mathrm{H}_{2}$, and MIR emission indicate that this SNR is interacting with molecular clouds (Reach et al. 2006; Zhu, Tian \& Zuo 2014; Kilpatrick et al. 2016), although this is not completely clear as opposing studies find a lack of evidence for molecular material around the SNR (Lacey et al. 2001; Ranasinghe \& Leahy 2018a).

Our analysis supports the argument that there is some interaction as we detect warm dust (up to $\sim 36 \mathrm{~K}$ ) which coincides with the expected location of interactions to the east and south-west.
We also detect warm $(\sim 29-32 \mathrm{~K})$ dust from a filament at $\alpha=$ $19^{\mathrm{h}} 11^{\mathrm{m}} 07^{\mathrm{s}}, \delta=+09^{\circ} 07^{\prime} 01^{\prime \prime}$ from which MIR colours suggest line emission from ionic shocks (Reach et al. 2006).

G65.8-0.5: We find evidence of warm dust ( $\sim 24-32 \mathrm{~K})$ towards the east of the remnant, at the location of the $\mathrm{H} \alpha$ structure discussed in Section 2.1. Herschel 160-500 $\mu \mathrm{m}$ emission was detected from a structure towards the radio centre of the SNR in Section 2.1, this region has temperatures between $\sim 20$ and $22 \mathrm{~K}$.

G67.8+0.5: There is some evidence of anticorrelation between the radio contours and the dust temperatures. A warm $(\sim 38 \mathrm{~K})$ region of dust lies in the region between two radio peaks towards the west of the remnant, as shown by the contours in Fig. 21. There is also a peak of warm dust near the centre of the SNR which is located in a gap in the brightest radio structure. The origin of the warm dust is unclear as there is limited observations of this source. If the material is associated with the SNR it could be warm ejecta material, reverse-shocked material, or evidence of dust destruction.

We do not see evidence of warm dust at the location of optical emission to the west, where there is a potential bow shock (Sabin et al. 2013).

Kes 20A, G310.8-0.4: Spitzer Infrared Spectrograph (IRS) observations to the south-east suggest that this SNR has a relatively low velocity shock $\left(\sim 35-70 \mathrm{~km} \mathrm{~s}^{-1}\right)$ into a low-density medium $\left(\sim 10^{3} \mathrm{~cm}^{-3}\right)$ and has a distribution of dust grains that indicates shattering of grains in the shock (Andersen et al. 2011). We do not see evidence for dust in the south-east region that is significantly warmer than the surrounding ISM. The warmest dust identified in this source is towards the north, at the location of FIR and radio structure. If this warm dust is associated with the SNR the correlation with radio could indicate shock-heated dust in this region.

G340.6+0.3: Elemental abundances indicate that ejecta dominates the X-ray bright northern shell, whereas the emission in the south is from shocked ISM (Mangano et al. 2014). We find evidence of warm dust $(\sim 25-30 \mathrm{~K})$ in the northern arc with a peak in the north-east, slightly offset from the X-ray contours, which may be warm ejecta dust. There is also warm dust in the south in a region of bright X-ray emission, although the warmest dust in this area is offset from the brightest X-ray structures. Because of the coincidence we expect that the warm dust is also shocked ISM.

G349.7+0.2: There is evidence from $\mathrm{OH}(1720 \mathrm{MHz})$ masers (Frail et al. 1996) (see the yellow diamonds in Fig. 21) and line emission (Reynoso \& Mangum 2000; Lazendic et al. 2010) that this SNR is interacting with a coincident molecular cloud (Dubner et al. 2004). Although there is bright Herschel emission towards the central interacting region, our analysis suggests that the warmest dust is offset to the east.

The SNR is expanding at $\sim 710 \mathrm{~km} \mathrm{~s}^{-1}$ (Lazendic et al. 2005) into a medium with a density gradient, where the density is relatively low towards the west and higher towards the east. Corresponding to this gradient, we find warmer material in the eastern region where the SNR is dominated by swept-up medium (Lazendic et al. 2005), whereas towards the west we do not see evidence for SNR material that is noticeably warmer than the surrounding ISM. It is likely that the warm dust in the east of the remnant is swept-up material.

This paper has been typeset from a $\mathrm{T}_{\mathrm{E}} \mathrm{X} / \mathrm{LT} \mathrm{E} \mathrm{X}$ file prepared by the author. 